\title{
Complex-scaling method for the complex plasmonic resonances of planar subwavelength particles with corners
}

\author{
Anne-Sophie Bonnet-Ben Dhia ${ }^{\mathrm{a}}$, Christophe Hazard ${ }^{\mathrm{a}}$, Florian Monteghettia,* \\ ${ }^{a}$ POEMS (CNRS-INRIA-ENSTA Paris), Institut Polytechnique de Paris, Palaiseau, France
}

\begin{abstract}
A subwavelength metallic particle supports localized surface plasmons for some negative permittivity values, which are eigenvalues of the self-adjoint quasi-static plasmonic eigenvalue problem (PEP). This work investigates the existence of complex plasmonic resonances for a 2D particle whose boundary is smooth except for one straight corner. These resonances are defined using the multivalued nature of some solutions of the corner dispersion relations and they are shown to be eigenvalues of a PEP that is complex-scaled at the corner, the finite element discretization of which yields a linear generalized eigenvalue problem. Numerical results show that the complex scaling deforms the essential spectrum (associated with the corner) so as to unveil both embedded plasmonic eigenvalues and complex plasmonic resonances. The later are analogous to complex scattering resonances with the local behavior at the corner playing the role of the behavior at infinity. These results corroborate the study of Li and Shipman (J. Integral Equ. Appl. 31(4), 2019), which proved the existence of embedded plasmonic eigenvalues and discussed the construction of particles that exhibit complex plasmonic resonances.
\end{abstract}

Keywords: plasmonics, Neumann-Poincaré operator, complex resonances, embedded eigenvalues, complex scaling, perfectly matched layer, 2010 PACS: 73.20.Mf, 2010 MSC: 35P99

(C) 2021. Licensed under CC BY-NC-ND 4.0. This is an author-prepared version of the accepted manuscript, which appeared in the "Journal of Computational Physics 440 (2021)", 10.1016/j.jcp.2021.110433.

\section{Introduction}

The broad focus of this paper is the quasi-static plasmonic eigenvalue problem (PEP) for planar domains with corners. We consider a subwavelength plasmonic particle $\Omega_{m}$ (typically made of metal), assumed to be a piecewisesmooth bounded open set, surrounded by a dielectric medium $\Omega_{d}:=\mathbb{R}^{2} \backslash \overline{\Omega_{m}}$. If $u$ denotes the electric field potential, then the strong form of the PEP is: find $(u, \kappa)$ such that

$$
\Delta u(x)=0 \quad\left(x \in \Omega_{m} \cup \Omega_{d}\right),
$$

with the transmission boundary conditions

$$
u_{\mid \Omega_{m}}(\boldsymbol{x})=u_{\mid \Omega_{d}}(\boldsymbol{x}), \partial_{n} u_{\mid \Omega_{m}}(\boldsymbol{x})=\kappa \partial_{n} u_{\mid \Omega_{d}}(\boldsymbol{x}) \quad\left(\boldsymbol{x} \in \partial \Omega_{m}\right),
$$

where $\boldsymbol{n}$ denotes a unit normal defined on $\partial \Omega_{m}$. In addition, $u$ must satisfy the decay condition at infinity

$$
u(x) \underset{|x| \rightarrow \infty}{=} O\left(|x|^{-1}\right),
$$

which excludes the trivial solutions $(u=$ cst, $\kappa)[1, \S 2.4]$.

The spectral parameter $\kappa$, known as the contrast, is the ratio of dielectric permittivity across the interface $\partial \Omega_{m}$. Physically $\kappa$ depends upon the angular frequency $\omega$, the simplest classical model being that of a free electron gas

\footnotetext{
${ }^{*}$ Corresponding author

Email address: florian.monteghetti@ensta-paris.fr (Florian Monteghetti)
} 


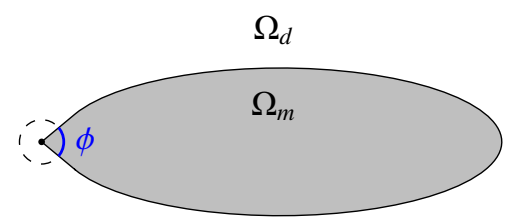

(a) Overview with corner neighborhood highlighted.

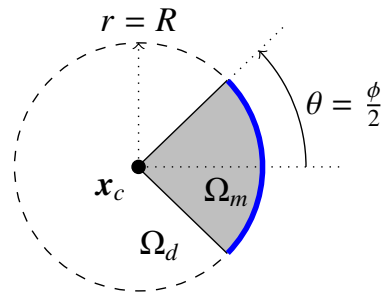

(b) Corner neighborhood $D:=\left\{\left|\boldsymbol{x}-\boldsymbol{x}_{c}\right|<R\right\}$.

Fig. 1: Particle $\Omega_{m}$ and dielectric medium $\Omega_{d}$. The sign-changing interface $\partial \Omega_{m}$ is smooth except for a straight corner of angle $\phi$.

known as Drude's model [2, Eq. (6.18)] [3, Eq. (7.56)]. However this dependency is not considered further herein since $\omega$ does not appear explicitly in the quasi-static approximation (1), which can be obtained as the leading-order approximation of a frequency-dependent problem (assuming the diameter of $\Omega_{m}$ is small compared to the wavelength) $[4,5]$. Solutions of the PEP $(1,2,3)$ are surface waves known as localized surface plasmons (abbreviated as "surface plasmons" for conciseness), whose energy-concentrating properties are commonly used today in label-free chemical and biological sensors with applications in areas as diverse as pollution monitoring, medical diagnostic, pharmaceutical development, and toxicology [6] [7, Chap. 11] [8]. Surface plasmons also have high-potential applications in photonics $[9,10]$, such as subwavelength or "perfect" lenses $[11, \S 3][12, \S 4]$.

The presence of corners in $\partial \Omega_{m}$ implies theoretical and numerical difficulties, which have been studied within different bodies of literature surveyed below.

\subsection{Analysis and discretization of the PEP weak formulation}

A formal integration by parts of the PEP $(1,2,3)$ yields: find $(u, \kappa)$ such that, for any test function $v$,

$$
\int_{\Omega_{m}} \nabla u(\boldsymbol{x}) \cdot \nabla v(\boldsymbol{x}) \mathrm{d} \boldsymbol{x}=-\kappa \int_{\Omega_{d}} \nabla u(\boldsymbol{x}) \cdot \nabla v(\boldsymbol{x}) \mathrm{d} \boldsymbol{x},
$$

which is a generalized eigenvalue problem. Taking $v=\bar{u}$ shows that $\kappa \leq 0$, i.e. the permittivity changes sign: the interface $\partial \Omega_{m}$ is said to be sign-changing. This complicates the analysis of well-posedness as well as discretization since ellipticity is lost (the associated bilinear form is coercive if and only if $\kappa \notin(-\infty, 0][13, \S 3])$. A crucial result is that the topology of the spectrum depends on the interface's smoothness. If $\partial \Omega_{m}$ is smooth then the spectrum consists of a sequence of distinct eigenvalues $\kappa_{n}$ that accumulates at -1 [1, Thm. 1]. The closer $\kappa_{n}$ is to -1 , the more oscillating and localized (at the surface) the corresponding eigenfunction is, as illustrated in Figure 9.

However, if the interface has a corner of angle $\phi \in(0, \pi)$ (as illustrated in Figure 1a), then the spectrum contains the critical interval $[14,13]$

$$
I_{c}=\left(-\frac{2 \pi-\phi}{\phi},-1\right) \cup\left(-1,-\frac{\phi}{2 \pi-\phi}\right) \subset(-\infty, 0),
$$

which can be interpreted as essential spectrum [15, Thm. 3] [16]. Each contrast $\kappa$ in the critical interval is associated with a pair of strongly-oscillating local corner solutions, conjugate of each other, depicted in Figure 2. A peculiar feature of these two local solutions is that they do not have finite energy (i.e. they belong to $L^{2}$ but not $H^{1}$ around the corner). Physical insights can be gained by working in the Euler coordinates $(z, \theta)$, deduced from the polar coordinates centered at the corner by $(z=\ln r, \theta)$, which send the corner $r=0$ to infinity $z=-\infty$ : the two local solutions can be interpreted as waves with opposite group velocities radiating energy either "to" or "from" the corner, each respecting a different radiation condition at $z \rightarrow-\infty$ [13]. The "outgoing" wave, which is the one needed to satisfy the limiting absorption principle, has been called a black-hole wave [13] since it effectively traps energy at the corner.

Numerically, this analysis has been used to design a perfectly matched layer (PML) that selects the desired wave at the corner. This corner PML has been demonstrated on a finite element (FE) discretization of the plasmonic scattering problem with $\kappa \in I_{c}$ in [17], where it is shown to yield a convergent discretization. In the physics literature, FE discretizations of $(1,2)$ without corner PML have been considered, see e.g. [18]. 


\subsection{Analysis and discretization of the PEP integral formulation (Neumann-Poincaré operator)}

By looking for $u$ as a single-layer potential $u=\mathcal{S}[\varphi]$, an integral formulation of the PEP $(1,2,3)$ can be obtained by combining the jump property [19, Thm. 3.28] [20, Chap.3] with the transmission conditions (2): find $(\varphi, \lambda)$ such that [21, Eq. (4)] [22, Thm. 2.1]

$$
\mathcal{K}^{\star} \varphi=\lambda \varphi
$$

where the operator $\mathcal{K}^{\star}$ is the adjoint of the double-layer potential, also known as the Neumann-Poincaré (NP) operator, and the link between the spectral parameter $\lambda$ and the contrast $\kappa$ is given by

$$
\lambda=\frac{1}{2} \times \frac{1+\kappa}{1-\kappa}, \kappa=\frac{2 \lambda-1}{2 \lambda+1} .
$$

The integral formulation of the non quasi-static case is derived in [23, Eq. (2.3)].

Numerically, boundary element (BE) methods have been used in the physics literature to solve the PEP, see [24] for instance. A detailed comparative study of BE methods for particles with corners is available in [25]. Theoretically, the NP operator has enjoyed a renewed scrutiny stimulated by the interest in plasmonics: see e.g. [26] for an optimal shape design problem, [4] for a mathematical study of surface plasmons, [5] for an investigation into the validity of the quasi-static approximation, [27] for a study of the rate of resonance, and [28] for a spectral resolution of the NP operator on intersecting disks.

Let us now review some results of spectral theory: in agreement with Section 1.1, the spectrum of the NP operator depends upon the smoothness of the interface. For a Lipschitz boundary, $\mathcal{K}^{\star}$ is a bounded self-adjoint operator. ${ }^{1}$ If the interface is smooth then $\mathcal{K}^{\star}$ is compact and its spectrum consists of a sequence of eigenvalues $\lambda_{n}$ that accumulates at $\lambda=0(\kappa=-1)$. If the interface has a corner of angle $\phi \in(0, \pi)$ then $\mathcal{K}^{\star}$ loses its compactness: its essential spectrum is given by [31, Thm. 7] (see also [32, Eq. (7)])

$$
\sigma_{\text {ess }}\left(\mathcal{K}^{\star}\right)=\left[\frac{\phi-\pi}{2 \pi}, \frac{\pi-\phi}{2 \pi}\right]=\left\{\lambda=\frac{1}{2} \times \frac{1+\kappa}{1-\kappa} \mid \kappa \in \overline{I_{c}}\right\} \subset\left(-\frac{1}{2}, \frac{1}{2}\right)
$$

and its eigenvalues $\lambda_{n}$ can only accumulate at 0 and $\pm \frac{\phi-\pi}{2 \pi}[33$, Thm. A].

A numerical investigation into the different components of $\sigma\left(\mathcal{K}^{\star}\right)$ using a rate-of-resonance criterion showed the presence of embedded eigenvalues for an elliptical particle perturbed by a corner [34]. It is proven in [35, Thm. 8] that given a symmetric closed $C^{2}$ interface, there exists a corner perturbation that generates embedded eigenvalues. Moreover, in [35, §5.2] it is suggested that this corner perturbation can lead to some eigenvalues crossing the essential spectrum to become complex resonances: this will be confirmed using complex scaling in Section 5.

\subsection{Outline}

The objective of this paper is to investigate the existence of so-called complex plasmonic resonances for a $2 \mathrm{D}$ subwavelength particle whose boundary is smooth except for one straight corner. We first propose a definition of complex plasmonic resonances that relies solely on an analysis of the corner dispersion relations (Definition 17). Complex plasmonic resonances are not eigenvalues of the PEP $(1,2,3)$, which makes their computation challenging. However we show that they can be computed as eigenvalues of a modified PEP, obtained using a corner complex scaling. A FE discretization of this complex-scaled PEP yields a complex-symmetric linear generalized eigenvalue problem.

In the footsteps of $[34,35]$, the numerical application focuses on an elliptical particle perturbed by a corner. Results show that the complex scaling deforms the critical interval $I_{c}$ so as to unveil both embedded plasmonic eigenvalues and complex plasmonic resonances. The latter are associated with surface plasmons (named herein complex plasmonic resonance functions) whose behavior at the corner $\boldsymbol{x}_{c}$ is

$$
u(\boldsymbol{x}) \underset{\left|\boldsymbol{x}-\boldsymbol{x}_{c}\right| \rightarrow 0}{=} \frac{1}{\left|\boldsymbol{x}-\boldsymbol{x}_{c}\right|^{\mathfrak{I}(\eta)}} O(1) \quad(\mathfrak{J}(\eta)>0),
$$

\footnotetext{
${ }^{1}$ When considered as an operator from $H^{-1 / 2}\left(\partial \Omega_{m}\right)$ to itself, equipped with the inner product induced by the single-layer potential $\mathcal{S}$ : this technical result is a consequence of Plemelj's symmetrization principle [29] [30, Thm. 2.5].
} 
where the exponent $\eta$ is linked to the contrast $\kappa$ through the corner dispersion relations. Complex plasmonic resonances are formally analogous to complex scattering resonances, with the local behavior at the corner playing the role of the behavior at infinity. These results corroborate the study [35], which proved the existence of embedded plasmonic eigenvalues and discussed the construction of particles that exhibit complex plasmonic resonances.

This paper is organized as follows. Section 2 defines complex plasmonic resonances. To compute them, we rely on a corner complex scaling that is recalled and analyzed in Section 3. Section 4 validates the proposed FE formulation and emphasizes the discretization challenges inherent to the sign-changing nature of the interface. Lastly, Section 5 gathers the numerical results. The proofs of some technical or known results are gathered in Appendices A, B, and D. Appendix $\mathrm{C}$ contains further comments on the definition of complex plasmonic resonances proposed herein.

Remark 1 (On the meaning of "complex resonance"). In this paper, the denomination "complex resonance" is used in the sense of $[36,37]$. The complex resonances defined and computed in this work are designated "complex plasmonic resonances" to distinguish them from "complex scattering resonances". This terminology will be defined in Section 2.2.3, but to avoid confusion let us emphasize now that "complex resonance" and "complex resonance function" are different concepts from "complex eigenvalue" and "eigenfunction". In the physical literature, "complex resonance functions" are sometimes also called "quasi-normal modes".

\section{Basics of corner plasmonics and definition of complex plasmonic resonances}

The purpose of this section is to define complex plasmonic resonances. Section 2.1 recalls the "trinity of corner plasmonics": the corner dispersion relations (7), the associated sets of exponents (8), and the critical interval (10). Section 2.2 then defines complex plasmonic resonances in Definition 17. The proposed definition is computational inasmuch as it relies explicitly on the asymptotic expansion at the corner, which will ease the introduction of the complex-scaling technique in Section 3.

Assumption. Throughout Sections 2 and 3 the particle $\Omega_{m}$ is a bounded open set with a boundary $\partial \Omega_{m}$ that is smooth except at $\boldsymbol{x}_{c}$, where there is a straight corner of angle $\phi \in(0,2 \pi) \backslash\{\pi\}$, see Figure 1a for an example.

Notation. The dielectric medium is $\Omega_{d}:=\mathbb{R}^{2} \backslash \overline{\Omega_{m}}$. Let

$$
D:=\left\{\left|\boldsymbol{x}-\boldsymbol{x}_{c}\right|<R\right\}, \Theta_{m}:=\left(-\frac{\phi}{2}, \frac{\phi}{2}\right), \Theta_{d}:=\left(-\pi,-\frac{\phi}{2}\right) \cup\left(\frac{\phi}{2}, \pi\right] .
$$

The radius $R>0$ is small enough so that $D$ only includes the straight corner. The polar coordinates $(r, \theta)$ with $\theta \in(-\pi, \pi]$ are centered at $x_{c}$, see the sketch of Figure 1b. The set $D_{m}:=\Omega_{m} \cap D$ (resp. $\left.D_{d}:=\Omega_{d} \cap D\right)$ is described by $r<R$ and $\theta \in \Theta_{m}$ (resp. $\theta \in \Theta_{d}$ ).

\subsection{Corner dispersion relations and critical interval}

To define complex plasmonic resonances we first "zoom at the corner", i.e. we carry out a local study near the corner by considering

$$
\left\{\begin{array}{l}
\Delta u(\boldsymbol{x})=0 \quad\left(\boldsymbol{x} \in D_{m} \cup D_{d}\right) \\
u_{\mid D_{m}}(\boldsymbol{x})=u_{\mid D_{d}}(\boldsymbol{x}), \partial_{n} u_{\mid D_{m}}(\boldsymbol{x})=\kappa \partial_{n} u_{\mid D_{d}}(\boldsymbol{x}) \quad\left(\boldsymbol{x} \in \partial D_{m} \cap \partial D_{d}\right),
\end{array}\right.
$$

where there is no boundary condition on $\partial D$. The solutions of the local transmission problem (6) are given in Proposition 5, which requires two definitions used throughout the work.

Definition 2 (Corner dispersion relations). Let $\phi \in(0,2 \pi)$. The even (resp. odd) corner dispersion relation is

$$
f_{\phi}^{\mathfrak{e}(\mathfrak{o})}(\eta, \kappa)=0,
$$

where $f_{\phi}^{\mathrm{e}(\mathfrak{p})}: \mathbb{C} \times \mathbb{C} \rightarrow \mathbb{C}$ is given by $f_{\phi}^{\mathrm{e}(\mathfrak{o})}(\eta, \kappa):=[1+\kappa] \sinh [\eta \pi]_{(+)}^{-}[1-\kappa] \sinh [\eta(\pi-\phi)]$.

Remark 3. The name "dispersion relation" for (7) is motivated by time-harmonic variants of the PEP, where $\kappa$ is explicitly considered as a function of frequency.

Definition 4 (Sets of exponents). Let $\phi \in(0,2 \pi)$. For any $\kappa \in \mathbb{C}$, the set of even (resp. odd) exponents $H_{\phi}^{\mathfrak{e}(\mathfrak{)})}(\kappa)$ and the set of exponents $H_{\phi}(\kappa)$ are defined as

$$
H_{\phi}^{\mathfrak{e}(\mathfrak{D})}(\kappa):=\left\{\eta \in \mathbb{C} \mid f_{\phi}^{\mathrm{e}(\mathfrak{l})}(\eta, \kappa)=0\right\}, H_{\phi}(\kappa):=H_{\phi}^{\mathrm{e}}(\kappa) \cup H_{\phi}^{\mathfrak{o}}(\kappa) .
$$


Proposition 5 (Local solutions). Let $\phi \in(0,2 \pi) \backslash\{\pi\}$ and $\kappa_{\phi}:=-\frac{2 \pi-\phi}{\phi}$. For any $\kappa \in \mathbb{C} \backslash\left\{\kappa_{\phi}\right\}$, the solutions of the local transmission problem (6) with separated variables can be split into two families $\left(u_{\eta}^{\mathrm{e}}\right)_{\eta \in H_{\phi}^{\mathrm{e}}(\kappa)}$ and $\left(u_{\eta}^{\mathrm{v}}\right)_{\eta \in H_{\phi}^{\mathrm{o}}(\kappa) \backslash\{0\}}$ defined as

$$
u_{\eta}^{\mathrm{e}(\mathfrak{D})}(r, \theta):=r^{i \eta} \times \Phi_{\eta}^{\mathrm{e}(\mathfrak{o})}(\theta) \quad\left(\eta \in H_{\phi}^{\mathrm{e}(\mathfrak{D})}(\kappa) \backslash\{0\}\right), u_{0}^{\mathrm{e}}(r, \theta):=[1+c \ln (r)] \times \Phi_{0}^{\mathrm{e}}(\theta),
$$

where the orthoradial functions $\Phi_{\eta}^{\mathrm{e}}$ and $\Phi_{\eta}^{\mathfrak{0}}$ are given by (A.1) and (A.2), respectively.

Proof. Using weak derivatives, (6) reads

$$
\operatorname{div}(\sigma \nabla u)=\frac{1}{r^{2}}\left[r \partial_{r}\left(\sigma(\kappa, \theta) r \partial_{r} u\right)+\partial_{\theta}\left(\sigma(\kappa, \theta) \partial_{\theta} u\right)\right]=0 \quad(r \in(0, R), \theta \in(-\pi, \pi]),
$$

with $\sigma(\kappa, \theta):=1 / \kappa \mathbb{1}_{\Theta_{m}}(\theta)+\mathbb{1}_{\Theta_{d}}(\theta)$. If we look for a solution with separated variables $u(r, \theta)=v(r) w(\theta)$ we get

$$
\frac{1}{v(r)} r \partial_{r}\left(r \partial_{r} v(r)\right)=-\eta^{2}=-\frac{1}{\sigma(\kappa, \theta) w(\theta)} \partial_{\theta}\left(\sigma(\kappa, \theta) \partial_{\theta} w(\theta)\right),
$$

where $\eta \in \mathbb{C}$ is a free parameter. Proposition 27 shows that the orthoradial equation is solvable if and only if $f_{\phi}^{\mathrm{e}}(\eta, \kappa)=0$ or $f_{\phi}^{\mathrm{o}}(\eta, \kappa)=0$. Since $\kappa \neq \kappa_{\phi}$, the odd piecewise-linear function $\Phi_{0}^{\mathrm{o}}$ cannot solve the orthoradial equation. The change of variables $z=\ln (r)$ enables to write the radial equation as $v^{\prime \prime}(z)=-\eta^{2} v(z)$ on $(-\infty, \ln R)$, which is readily solvable for any $\eta \in \mathbb{C}$.

The regularity of these local solutions at the corner is driven by the sign of $\mathfrak{J}(\eta)$ :

- If $\mathfrak{J}(\eta)<0$, then $u_{\eta}^{\mathrm{e}(\mathfrak{o})}$ vanishes at the corner and $u_{\eta}^{\mathrm{e}(\mathfrak{o})} \in H^{1}(D)$.

- If $\mathfrak{J}(\eta)>0$ then $u_{\eta}^{\mathrm{e}(\mathfrak{o})}$ is singular at the corner and $u_{\eta}^{\mathrm{e}(\mathfrak{o})} \notin H^{1}(D)$. The modulus of $u_{\eta}^{\mathrm{e}(\mathfrak{o})}$ tends to infinity as $r \rightarrow 0$. Moreover, if $\mathfrak{J}(\eta)>1$ then $u_{\eta}^{\mathrm{e}(\mathfrak{o})} \notin L^{2}(D)$.

- If $\mathfrak{J}(\eta)=0$, then there are three cases to distinguish:

- If $\eta \in \mathbb{R}^{*}$ then $u_{\eta}^{\mathrm{e}(\mathfrak{o})}(r, \theta)=e^{i \eta \ln r} \Phi_{\eta}^{\mathrm{e}(\mathfrak{)})}(\theta) \in L^{2}(D) \backslash H^{1}(D)$ is a (bounded) strongly-oscillating function (in $r$ ) that does not have a limit at $r=0$.

- If $\eta=0$ and $c=0$, then $u_{0}^{\mathrm{e}} \in H^{1}(D)$ since $u_{0}^{\mathrm{e}}(r, \theta)=1$.

- If $\eta=0$ and $c \neq 0$, then $u_{0}^{\mathrm{e}} \in L^{2}(D) \backslash H^{1}(D)$ due to its logarithmic singularity.

Since we will use these local solutions to expand the solution of the PEP $(1,2,3)$ in $D$, it will prove useful to know the content of $H_{\phi}(\kappa)$ for a given contrast $\kappa$ and corner angle $\phi$ : Lemma 6 covers elementary properties while Proposition 7 covers the strongly-oscillating case $\eta \in \mathbb{R}^{*}$.

Lemma 6 (Elementary properties). Let $\kappa \in \mathbb{C}$ and $\phi \in(0,2 \pi) \backslash\{\pi\}$. The following properties hold:

(a) $H_{\phi}^{\mathrm{e}(\mathfrak{)})}(\kappa)$ is countable and has no finite accumulation point.

(b) If $\eta \in H_{\phi}^{\mathrm{e}(\mathfrak{o})}(\kappa)$, then $-\eta \in H_{\phi}^{\mathrm{e}(\mathfrak{o})}(\kappa)$ and $\bar{\eta} \in H_{\phi}^{\mathrm{e}(\mathfrak{o})}(\bar{\kappa})$.

(c) $0 \in H_{\phi}^{\mathrm{e}(\mathrm{p})}(\kappa)$ and $H_{\phi}^{\mathrm{e}}(\kappa) \cap H_{\phi}^{\mathrm{o}}(\kappa) \subset i \mathbb{R}$.

Proof. See Appendix B.1.

The next result shows that the strongly-oscillating case $\eta \in \mathbb{R}^{*}$ can only occur when $\kappa$ belongs to the so-called critical interval $I_{c}:[17$, Eq. (10)]

$$
I_{c}:=I_{c}^{\mathrm{o}} \cup I_{c}^{\mathrm{e}}, I_{c}^{\mathrm{o}}:=\left\{\begin{array}{ll}
\left(\kappa_{\phi},-1\right) & (0<\phi<\pi) \\
\left(-1, \kappa_{\phi}\right) & (\pi<\phi<2 \pi)
\end{array}, I_{c}^{\mathrm{e}}:= \begin{cases}\left(-1, \kappa_{\phi}^{-1}\right) & (0<\phi<\pi) \\
\left(\kappa_{\phi}^{-1},-1\right) & (\pi<\phi<2 \pi) .\end{cases}\right.
$$

Proposition 7 (Critical interval). Let $\phi \in(0,2 \pi) \backslash\{\pi\}$. We have the equivalences

$$
\kappa \in I_{c}^{\mathrm{e}(\mathfrak{o})} \Leftrightarrow H_{\phi}^{\mathrm{e}(\mathfrak{l})}(\kappa) \cap \mathbb{R}^{*} \neq \varnothing \Leftrightarrow \exists ! \eta_{c}>0: H_{\phi}^{\mathrm{e}(\mathfrak{p})}(\kappa) \cap \mathbb{R}^{*}=\left\{\eta_{c},-\eta_{c}\right\},
$$

where the scalar $\eta_{c}$ is the critical exponent. Moreover, $\eta_{c}$ crosses the real axis when $\kappa$ crosses $I_{c}^{\mathrm{e}(\mathfrak{o})}$. 

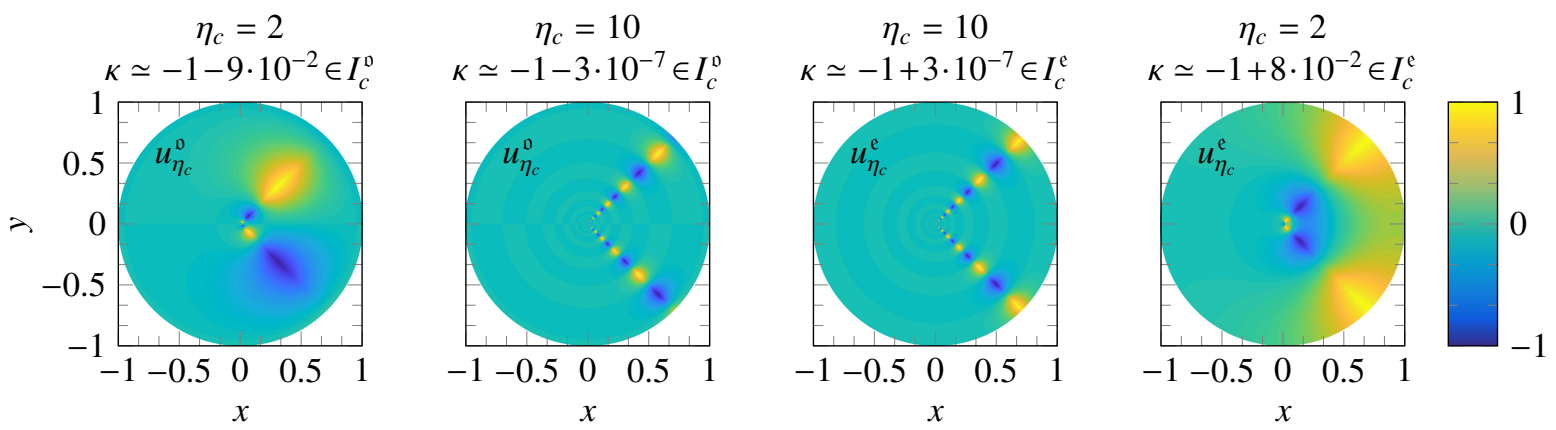

Fig. 2: Strongly-oscillating local solutions $\mathfrak{R}\left(u_{\eta_{c}}^{\mathrm{e}(\mathfrak{D})}\right) /\left\|u_{\eta_{c}}^{\mathrm{e}(\mathfrak{D})}\right\|_{\infty}$ given by (12) for a corner angle $\phi=\pi / 2$. The contrast $\kappa$ solves $f_{\phi}^{\mathrm{e}(\mathfrak{D})}\left(\eta_{c}, \kappa\right)=0$.

Proof. Let

$$
\psi_{\phi}(\eta):=\frac{\sinh [\eta(\pi-\phi)]}{\sinh [\eta \pi]}, \beta(x):=\frac{x-1}{x+1} .
$$

The smooth even map $\psi_{\phi}$ is strictly monotonous on $(0, \infty)$, since $\psi_{\phi}^{\prime}(\eta)=0$ is equivalent to $a \tanh [\eta \pi]=\tanh [a \eta \pi]$ with $a=1-\phi / \pi . \psi_{\phi}$ maps $(0, \infty)$ onto $\left(\frac{\pi-\phi}{\pi}, 0\right)$ if $\phi \in(\pi, 2 \pi)$ and onto $\left(0, \frac{\pi-\phi}{\pi}\right)$ if $\phi \in(0, \pi)$. The claimed equivalences follow from $f_{\phi}^{\mathrm{e}}(\eta, \kappa)=0 \Leftrightarrow \kappa=\beta \circ \psi_{\phi}(\eta)$ and $f_{\phi}^{\mathrm{o}}(\eta, \kappa)=0 \Leftrightarrow 1 / \kappa=\beta \circ \psi_{\phi}(\eta)$, where $\beta$ is an increasing map from $(-1,1)$ onto $(-\infty, 0)$. The last claim follows from the implicit function theorem and is proven in Appendix B.1.

When the contrast is critical, i.e. when $\kappa \in I_{c}^{\mathrm{e}(\mathfrak{o})}$, the two strongly-oscillating functions

$$
u_{\eta_{c}}^{\mathrm{e}(\mathfrak{o})}(r, \theta)=r^{i \eta_{c}} \Phi_{\eta_{c}}^{\mathrm{e}(\mathfrak{o})}(\theta), u_{-\eta_{c}}^{\mathrm{e}(\mathfrak{p})}(r, \theta)=r^{-i \eta_{c}} \Phi_{\eta_{c}}^{\mathrm{e}(\mathfrak{D})}(\theta)=\overline{u_{\eta_{c}}^{\mathrm{e}(\mathfrak{)})}}(r, \theta) \quad(r \in(0, R), \theta \in(-\pi, \pi])
$$

solve (6). These functions do not have a limit when $r \rightarrow 0$. As $\kappa \in I_{c}$ tends to $\kappa_{\phi}$ or $1 / \kappa_{\phi}$, the (real) critical exponent $\eta_{c}$ tends to 0 , while as $\kappa \rightarrow-1, \eta_{c}$ goes to infinity.

Remark 8. As $\phi \rightarrow \pi$ (locally flat interface), the closure of the critical interval reduces to $\{-1\}$. The closure of the critical interval can be interpreted as the essential spectrum of a suitably defined operator $[15,31,16]$.

The results covered so far apply to any corner angle $\phi \neq \pi$ and constitute all that is needed to introduce complex plasmonic resonances in Section 2.2. The remainder of this section focuses on the case $\phi / \pi \in \mathbb{Q}$, for which the knowledge of $H_{\phi}$ is reduced to that of a bounded subset $\breve{H}_{\phi}$ that can be obtained by solving a polynomial equation.

Proposition 9. Let $\kappa \in \mathbb{C} \backslash\{-1\}$. If $\phi \in(0,2 \pi) \backslash\{\pi\}$ and $\phi / \pi=p / q$ with $p$ and $q$ coprime integers, then the set of exponents can be split into a $\kappa$-independent and a $\kappa$-dependent part:

$$
H_{\phi}^{\mathrm{e}(\mathfrak{p})}(\kappa)=[i q \mathbb{Z}] \cup\left[\breve{H}_{\phi}^{\mathrm{e}(\mathfrak{o})}(\kappa)+i 2 q \mathbb{Z}\right],
$$

where $\breve{H}_{\phi}^{\mathrm{e}(\mathfrak{o})}(\kappa) \subset\{\eta \in \mathbb{C} \mid-q<\mathfrak{J}(\eta) \leq q\}$ contains at most $2(q-1)$ distinct elements and satisfies

$$
\breve{H}_{\phi}^{\mathrm{e}}(\kappa)=\left\{\frac{q}{\pi} \ln (x) \mid P_{\beta(\kappa)}^{(p, q)}(x)=0\right\}, \breve{H}_{\phi}^{\mathrm{o}}(\kappa)=\left\{\frac{q}{\pi} \ln (x) \mid P_{-\beta(\kappa)}^{(p, q)}(x)=0\right\},
$$

where the polynomial $P_{\beta(\kappa)}^{(p, q)}$ is given by (B.1) and $\ln$ denotes the principal branch of the logarithm.

Proof. The proof consists in formulating a polynomial equation in $x=e^{\frac{\eta \pi}{q}}$, see Appendix B.2.

For a right angle $\phi=\pi / 2$, we have (see Example 29 for details)

$$
\breve{H}_{\phi}^{\mathrm{e}}(\kappa)=\left\{\eta_{\beta(\kappa)}^{+},-\eta_{\beta(\kappa)}^{+}\right\}, \breve{H}_{\phi}^{\mathrm{v}}(\kappa)=\left\{\eta_{-\beta(\kappa)}^{+},-\eta_{-\beta(\kappa)}^{+}\right\},
$$

where the exponents, which only depend on $\kappa$ through $\beta(\kappa)$, are given by:

$$
\eta_{\beta(\kappa)}^{+}:=\frac{2}{\pi} \ln \left[\frac{-\beta(\kappa)+\sqrt{\beta(\kappa)^{2}-4}}{2}\right], \eta_{-\beta(\kappa)}^{+}:=\frac{2}{\pi} \ln \left[\frac{\beta(\kappa)+\sqrt{\beta(\kappa)^{2}-4}}{2}\right],
$$



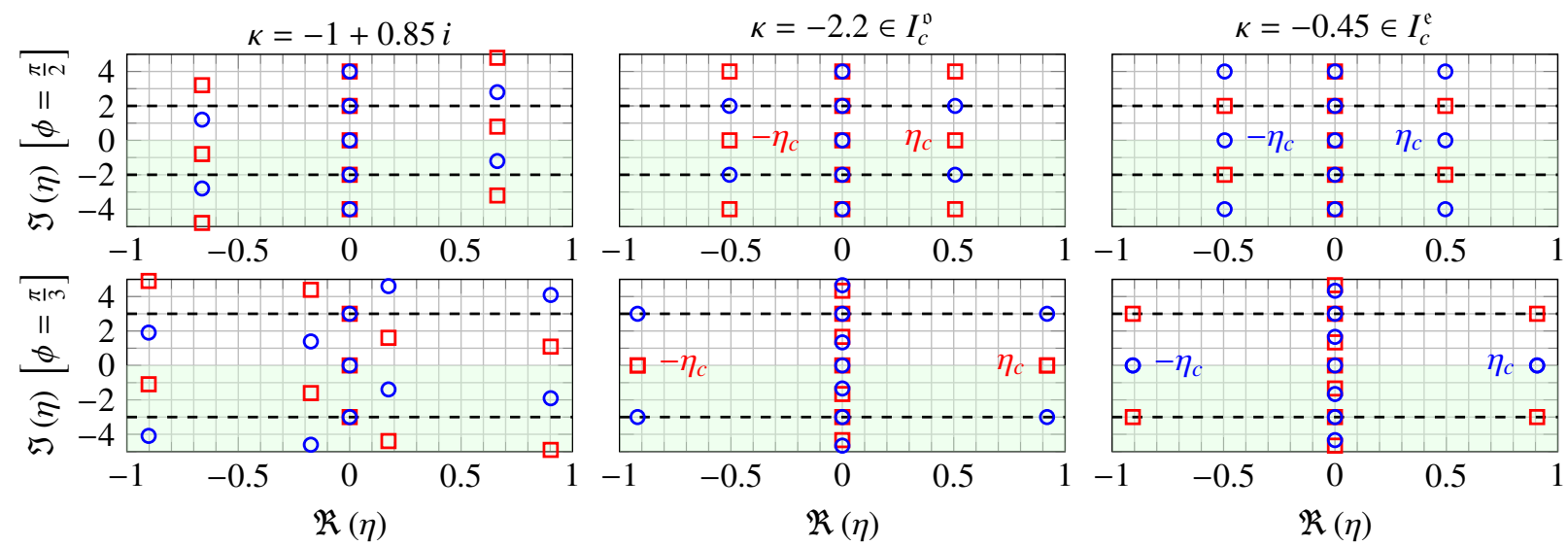

Fig. 3: Sets of exponents, computed using Proposition 9. $(\bigcirc): H_{\phi}^{\mathrm{e}}(\kappa),(\square)$ : $H_{\phi}^{\mathrm{p}}(\kappa)$. The stable region $\{\mathfrak{J}(\eta)<0\}$ is shaded. (Top row) $\phi=\pi / 2$, for which $\breve{H}_{\phi}^{\mathrm{e}(\mathfrak{o})}(\kappa) \subset\{\eta \in \mathbb{C} \mid-2<\mathfrak{J}(\eta) \leq 2\}$. (Bottom row) $\phi=\pi / 3$, for which $\breve{H}_{\phi}^{\mathrm{e}(\mathfrak{o})}(\kappa) \subset\{\eta \in \mathbb{C} \mid-3<\mathfrak{J}(\eta) \leq 3\}$.

using the principal branches of both $\ln$ and $\sqrt{\cdot}$. In agreement with Proposition 7 , we have:

$$
\kappa \in I_{c}^{\mathrm{e}}=(-1,-1 / 3) \Leftrightarrow \beta(\kappa) \in(-\infty,-2) \Leftrightarrow \eta_{\beta(\kappa)}^{+}(\kappa)>0, \kappa \in I_{c}^{\mathrm{D}}=(-3,-1) \Leftrightarrow \beta(\kappa) \in(2,+\infty) \Leftrightarrow \eta_{-\beta(\kappa)}^{+}(\kappa)>0 .
$$

The associated strongly-oscillating local solutions are plotted in Figure 2. The case $\phi=\pi / 3$ is similar:

$$
\breve{H}_{\phi}^{\mathrm{e}}(\kappa)=\left\{\eta_{\beta(\kappa)}^{+,+}, \eta_{\beta(\kappa)}^{+,-},-\eta_{\beta(\kappa)}^{+,+},-\eta_{\beta(\kappa)}^{+,-}\right\}, \breve{H}_{\phi}^{\mathrm{v}}(\kappa)=\left\{\eta_{-\beta(\kappa)}^{+,+}, \eta_{-\beta(\kappa)}^{+,-},-\eta_{-\beta(\kappa)}^{+,+},-\eta_{-\beta(\kappa)}^{+,-}\right\}
$$

see Example 30 for details. Using these results Figure 3 plots the exponents for $\phi=\pi / 2$ and $\phi=\pi / 3$, highlighting the structure (13). It also shows the two real critical exponents $\pm \eta_{c}$ that are obtained when $\kappa \in I_{c}$.

\subsection{From multivalued exponents to complex plasmonic resonances}

We denote by $\mathbb{C}^{+}$and $\mathbb{C}^{-}$the open upper and lower half-spaces

$$
\mathbb{C}^{+}:=\{z \in \mathbb{C} \mid \mathfrak{I}(z)>0\}, \mathbb{C}^{-}:=\{z \in \mathbb{C} \mid \mathfrak{J}(z)<0\} .
$$

Let $\kappa_{0} \in \mathbb{C}^{+}$and $u$ be a $H^{1}(D)$ solution of the local transmission problem (6) with $\kappa=\kappa_{0}$. From the properties gathered in Section 2.1, notably the discussion that followed Proposition 5, $u$ admits the pointwise-convergent asymptotic expansion

$$
u(r, \theta) \underset{r \rightarrow 0}{=} c_{0}+\sum_{\rho \in\{e, 0\}} \sum_{\substack{\eta \in \widehat{H}_{\phi}^{\rho}\left(\kappa_{0}\right) \\ \eta_{\star}<\mathcal{S}(\eta)}} c_{\eta}^{\rho} u_{\eta}^{\rho}(r, \theta)+O\left(r^{-\eta_{\star}}\right) \quad(r \in(0, R), \theta \in(-\pi, \pi])
$$

for any $\eta_{\star}<0$, where the sets of so-called stable exponents are defined for any $\kappa \in \mathbb{C}^{+}$as

$$
\widehat{H}_{\phi}^{\mathrm{e}(\mathfrak{p})}(\kappa):=\left\{\eta \in H_{\phi}^{\mathrm{e}(\mathfrak{p})}(\kappa) \mid \mathfrak{I}(\eta)<0\right\}, \widehat{H}_{\phi}(\kappa):=\widehat{H}_{\phi}^{\mathrm{e}}(\kappa) \cup \widehat{H}_{\phi}^{\mathfrak{j}}(\kappa) \quad\left(\kappa \in \mathbb{C}^{+}\right) .
$$

A rigorous derivation of (16) could be carried out by inverting the Mellin transform of $u$ using the residue theorem, see $[38, \S 3.5]$ as well as $[39, \S 1.2][40$, Chap. 2] [41, § 2] [42, Chap. 2].

The purpose of this section is to construct a definition of complex plasmonic resonances by continuing $\kappa \mapsto \widehat{H}_{\phi}(\kappa)$, well-defined for any $\kappa \in \mathbb{C}^{+}$, to $\mathbb{C}^{-}$. The construction proceeds in three steps. Section 2.2.1 shows that $\widehat{H}_{\phi}$ has three branch points given by $\kappa=\kappa_{\phi}, \kappa=1 / \kappa_{\phi}$, and $\kappa=-1$. Section 2.2.2 introduces three different analytic continuations to $\mathbb{C}^{-}$, depending on where $\kappa$ crosses the real axis. This leads in Section 2.2.3 to the definition of distinct families of contrasts: isolated plasmonic eigenvalues, embedded plasmonic eigenvalues, and complex plasmonic resonances. 


\subsubsection{Branch points of the set of stable exponents}

Before tackling the general case it is instructive to highlight the multivalued nature of the set of stable exponents $\widehat{H}_{\phi}^{\mathrm{e}(\mathfrak{D})}$ when $\phi=\pi / 2$, since for this corner angle the exponents are known in closed form $(13,14,15)$.

Example $10($ Case $\phi=\pi / 2)$. The exponent $\eta_{-\beta(\kappa)}^{+}$is null only for $\kappa=-3(\beta(\kappa)=2)$ and is singular only at $\kappa=-1$ $(|\beta(\kappa)|=\infty)$. These two values are in fact algebraic and logarithmic branch points, respectively, as revealed by the asymptotic expansions

$$
\eta_{-\beta(\kappa)}^{+} \underset{\kappa \rightarrow-3}{=} \frac{2}{\pi} \sqrt{\beta(\kappa)-2}+O(|\beta(\kappa)-2|), \exp \left(\eta_{-\beta(\kappa)}^{+} \frac{\pi}{2}\right) \underset{\kappa \rightarrow-1}{=} \beta(\kappa)-\frac{1}{\beta(\kappa)}+O\left(\frac{1}{|\beta(\kappa)|^{3}}\right) .
$$

These branch points imply that $\kappa \mapsto \eta_{-\beta(\kappa)}^{+}$is a multivalued map, i.e. it admits distinct branches [43, § I.4-I.7]. When $\kappa$ circles once around $-3, \beta(\kappa)$ loops around 2 and the exponents $\eta_{-\beta(\kappa)}^{+}$and $-\eta_{-\beta(\kappa)}^{+}$swap places, each crossing the real axis once. When $\kappa$ circles once around $-1, \eta_{-\beta(\kappa)}^{+}$gets translated by $(i 2 \pi) \times \frac{2}{\pi}=i 4$ or $-i 4$, depending on the direction of rotation. Let us now investigate what the multivaluation of the exponent $\kappa \mapsto \eta_{-\beta(\kappa)}^{+}$implies for the sets of exponents defined so far:

- Let $\kappa \in \mathbb{C} \backslash\{-1\}$. The set $\breve{H}_{\phi}^{\mathrm{o}}(\kappa)+i 4 \mathbb{Z}$ contains all the possible values for $\eta_{-\beta(\kappa)}^{+}$and $-\eta_{-\beta(\kappa)}^{+}$, so the set-valued map $\kappa \mapsto \breve{H}_{\phi}^{\mathrm{o}}(\kappa)+i 4 \mathbb{Z}$ is not multivalued (i.e. starting at $\kappa$, regardless of the closed path one follows in the $\kappa$-plane one will never obtain two different sets at $\kappa$ ). A fortiori, the map $\kappa \mapsto H_{\phi}^{\mathfrak{j}}(\kappa)$ is not multivalued (see (13)). This illustrates that although some elements of $\kappa \mapsto H_{\phi}^{\mathfrak{j}}(\kappa)$ are multivalued maps, the set-valued map $\kappa \mapsto H_{\phi}^{\mathfrak{j}}(\kappa)$ is not multivalued (since it includes all the possible branches of its elements). (Note that not all elements of $\kappa \mapsto H_{\phi}^{\mathfrak{j}}(\kappa)$ are multivalued, since (13) implies that some elements of $H_{\phi}^{\mathfrak{o}}(\kappa)$ do not depend upon $\kappa$.)

- Let $\kappa \in \mathbb{C}^{+}$. The set $\widehat{H}_{\phi}^{\mathrm{v}}(\kappa)$ contains either $\eta_{-\beta(\kappa)}^{+}$or $-\eta_{-\beta(\kappa)}^{+}$but not both, so $\kappa=-3$ is an algebraic branch point of $\kappa \mapsto \widehat{H}_{\phi}^{\mathrm{v}}(\kappa)$. Moreover, $\widehat{H}_{\phi}^{\mathrm{v}}(\kappa)$ does not contain $\eta_{-\beta(\kappa)}^{+}+i 4$ or $-\eta_{-\beta(\kappa)}^{+}+i 4$ (since they have positive imaginary parts), so $\kappa=-1$ is a logarithmic branch point of $\kappa \mapsto \widehat{H}_{\phi}^{\mathrm{o}}(\kappa)$. Here, the multivaluation of some elements of $\kappa \mapsto \widehat{H}_{\phi}^{\mathrm{v}}(\kappa)$ does imply that the set-valued map $\kappa \mapsto H_{\phi}^{\mathrm{o}}(\kappa)$ is multivalued (since it does not include all the possible branches of its elements).

The even case is deduced with the substitution $(\beta(\kappa),-3, \mathfrak{v}) \rightarrow(-\beta(\kappa),-1 / 3, \mathfrak{e})$.

In the general case no closed form expression is available for the exponents. However the next result shows that if $\kappa \notin\left\{\kappa_{\phi}, 1 \kappa_{\phi}\right\}$, then $H_{\phi}^{\mathfrak{e}(\mathfrak{D})}(\kappa)$ consists only of simple zeros of $f_{\phi}^{\mathrm{e}(\mathfrak{D})}(\cdot, \kappa)$ that depend analytically on $\kappa$.

Proposition 11. Let $\phi \in(0,2 \pi) \backslash\{\pi\}$ and $\kappa_{0} \in U:=\mathbb{C} \backslash\left\{\kappa_{\phi}, 1 / \kappa_{\phi}\right\}$. For any $\eta_{0} \in H_{\phi}^{\mathfrak{e}(\mathfrak{D})}\left(\kappa_{0}\right)$, there are a neighborhood $V \subset U$ of $\kappa_{0}$ and a unique analytic map $\tilde{\eta}: V \rightarrow \mathbb{C}$ such that $\tilde{\eta}\left(\kappa_{0}\right)=\eta_{0}$ and $\tilde{\eta}(\kappa) \in H_{\phi}^{\mathrm{e}(\mathfrak{o})}(\kappa)$ for any $\kappa \in V$.

Proof. Consequence of the implicit function theorem [44, Prop. 2.14], see Appendix B.1.

Note that the above proposition does apply to $\kappa=-1$, as $H_{\phi}^{\mathrm{e}(\mathfrak{o})}(-1)=i \frac{\pi}{\pi-\phi} \mathbb{Z}$ is well-defined. However, the value $\kappa=-1$ is a singularity in the sense that some elements of $H_{\phi}^{\mathrm{e}(\mathfrak{D})}(\kappa)$ go to infinity when $\kappa \rightarrow-1$ (e.g. the critical exponent $\eta_{c}$ ). Therefore from now on the map $\kappa \mapsto H_{\phi}^{\mathrm{e}(\mathfrak{o})}(\kappa)$ is always considered defined on the open subset

$$
K:=\mathbb{C} \backslash\left\{\kappa_{\phi}, \frac{1}{\kappa_{\phi}},-1\right\} .
$$

Although the map $\kappa \mapsto H_{\phi}^{\mathrm{e}(\mathfrak{o})}(\kappa)$ is single-valued we have the following result, which generalizes the observations made in Example 10.

Proposition 12. Let $\phi \in(0,2 \pi) \backslash\{\pi\}$. The map $\mathbb{C}^{+} \ni \kappa \mapsto \widehat{H}_{\phi}^{\mathrm{e}(\mathfrak{)})}(\kappa)$ defined by (17) has one algebraic branch point at $\kappa=1 / \kappa_{\phi}\left(\right.$ resp. $\left.\kappa=\kappa_{\phi}\right)$ and one logarithmic branch point at $\kappa=-1$.

Proof. Follows from asymptotic expansions of the critical exponent, as in Example 10, see Appendix B.1.

The multivalued nature of the set-valued map $\kappa \mapsto \widehat{H}_{\phi}^{\mathrm{e}(\mathfrak{)})}(\kappa)$ is relied upon in the next sections to define complex plasmonic resonances. 


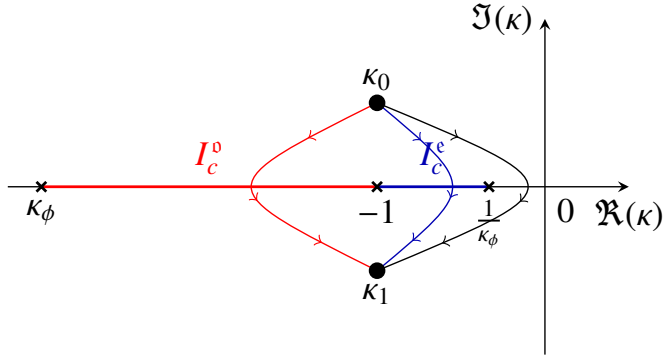

(a) $0<\phi<\pi$.

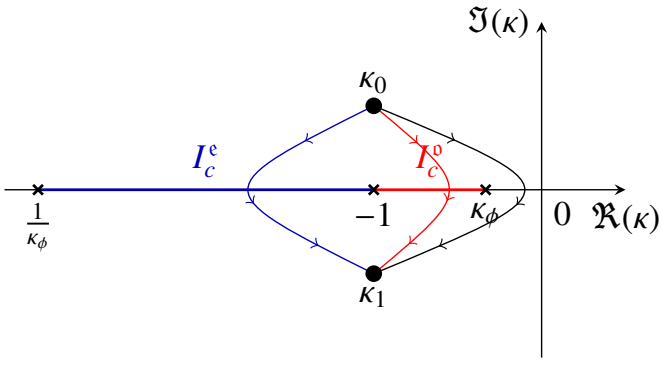

(b) $\pi<\phi<2 \pi$.

Fig. 4: Complex paths satisfying (18), each crossing the real axis at a different location: $\Gamma_{\phi}$ crosses outside the closure of the critical interval $\overline{I_{c}}, \Gamma_{\phi}^{\mathrm{e}}$ crosses through $I_{c}^{\mathfrak{e}}$, and $\Gamma_{\phi}^{\mathfrak{o}}$ crosses through $I_{c}^{\mathfrak{D}}$. Each path leads to a different continuation of $\mathbb{C}^{+} \ni \kappa \mapsto \widehat{H}_{\phi}(\kappa)$, see Figure 5 .

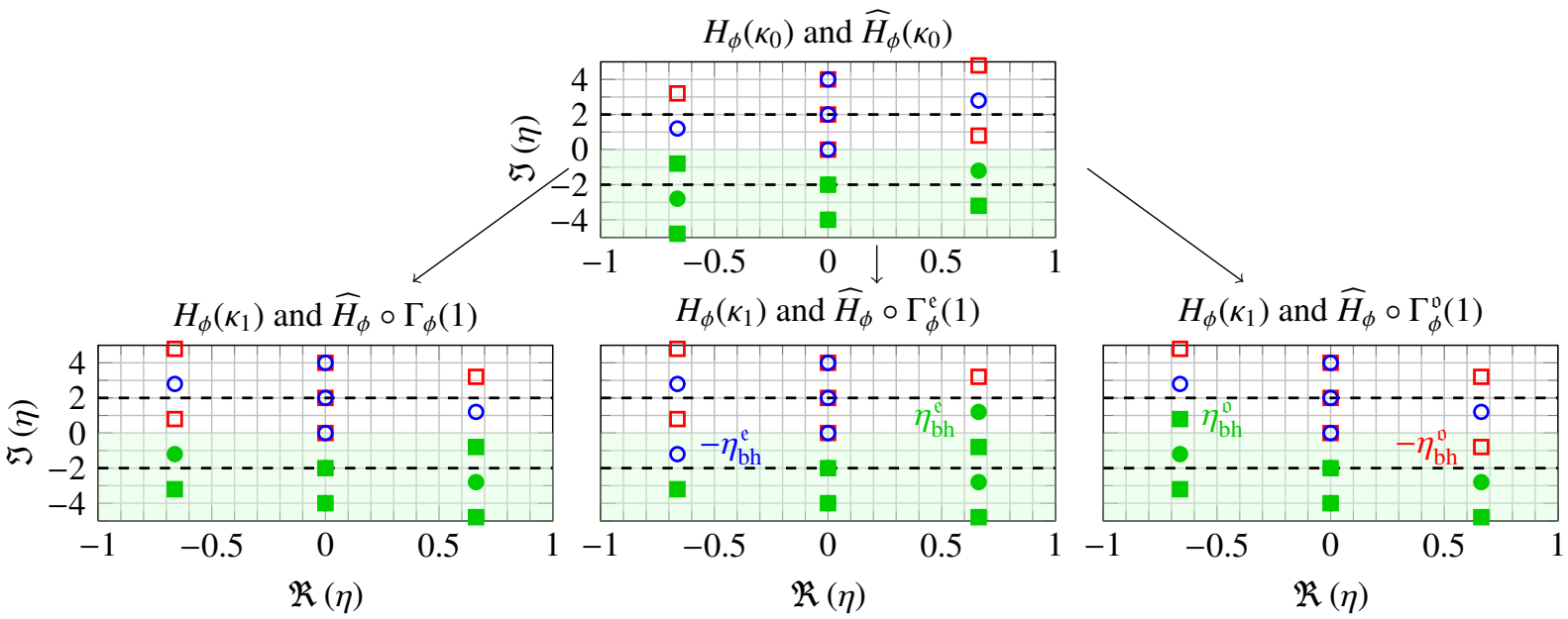

Fig. 5: Analytic continuation of $\kappa \mapsto \widehat{H}_{\phi}(\kappa)$ along each path of Figure 4, with $\phi=\pi / 2, \kappa_{0}=-1+0.85 i \in \mathbb{C}^{+}$, and $\kappa_{1}=\overline{\kappa_{0}}$. $(\bigcirc, \odot): H_{\phi}^{\mathrm{e}}(\kappa)$,

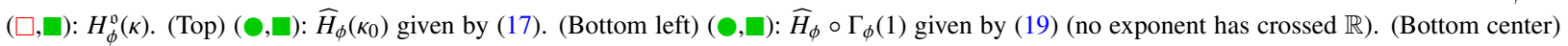
$(, \square): \widehat{H}_{\phi} \circ \Gamma_{\phi}^{\mathrm{e}}(1)$ given by (21) (the pair $\pm \eta_{\mathrm{bh}}^{\mathrm{e}}$ has crossed $\left.\mathbb{R}\right)$. (Bottom right) $(\bullet, \square): \widehat{H}_{\phi} \circ \Gamma_{\phi}^{\mathfrak{j}}(1)$ given by (23) (the pair $\pm \eta_{\mathrm{bh}}^{\mathrm{v}}$ has crossed $\left.\mathbb{R}\right)$.

\subsubsection{Analytic continuations of the set of stable exponents}

Intuitively, we would like to track the elements of $\widehat{H}_{\phi}(\kappa)$ as $\kappa$ travels from $\kappa_{0} \in \mathbb{C}^{+}$to the lower half-plane $\mathbb{C}^{-}$. To achieve this, we define a smooth complex path $\Gamma$ that satisfies

$$
\Gamma:(0,1) \rightarrow \mathbb{C}, \Gamma(0)=\kappa_{0}, \Gamma(1)=\kappa_{1},
$$

where $\kappa_{1}$ is an arbitrary contrast in $\mathbb{C}^{-}$. Proposition 11 implies that each element of $H_{\phi}\left(\kappa_{0}\right)$ can be considered as an analytic map defined in some neighborhood of $\kappa_{0}$; "tracking" an element of $H_{\phi}\left(\kappa_{0}\right)$ means building the analytic continuation of this map along $\Gamma$. Figure 4 sketches the three paths considered, which differ by where the real axis is crossed: $\Gamma_{\phi}$ crosses the real axis outside the closure of the critical interval $\overline{I_{c}}$, while $\Gamma_{\phi}^{\mathrm{e}}\left(\right.$ resp. $\Gamma_{\phi}^{\mathrm{o}}$ ) crosses the real axis through the even (resp. odd) critical interval $I_{c}^{\mathrm{e}}\left(\right.$ resp. $\left.I_{c}^{\mathrm{p}}\right)$. The top graph of Figure 5 plots $\widehat{H}_{\phi}\left(\kappa_{0}\right)$ for $\phi=\pi / 2$.

Crossing through $\mathbb{R} \backslash \overline{I_{c}}$. Let us first consider the behavior of $\widehat{H}_{\phi}$ along $\Gamma_{\phi}$. Proposition 7 gives that for any $s \in[0,1]$, $\widehat{H}_{\phi} \circ \Gamma_{\phi}(s)$ never contains a real element. In other words, no element of $\widehat{H}_{\phi}(\kappa)$ crosses the real axis as $\kappa$ travels along $\Gamma_{\phi}$. This provides us with a first value for " $\widehat{H}_{\phi}\left(\kappa_{1}\right)$ ", namely

$$
\widehat{H}_{\phi} \circ \Gamma_{\phi}(1)=\left\{\eta \in H_{\phi}\left(\kappa_{1}\right) \mid \mathfrak{I}(\eta)<0\right\},
$$

which is the one we could have expected at first sight. The bottom left graph of Figure 5 plots $\widehat{H}_{\phi} \circ \Gamma_{\phi}(1)$. 
Crossing through $I_{c}^{\mathfrak{e}}$. The main difference between $\Gamma_{\phi}$ and the even-crossing path $\Gamma_{\phi}^{\mathfrak{e}}$ follows from Proposition 7: when $\Gamma_{\phi}^{\mathrm{e}}(s)$ reaches $I_{c}^{\mathrm{e}}$ coming from $\mathbb{C}^{+}$, two non-null elements of $H_{\phi}^{\mathrm{e}} \circ \Gamma_{\phi}^{\mathrm{e}}(s)$ become real, namely $\pm \eta_{c}$. One of them, defined as the black-hole exponent $\eta_{\mathrm{bh}}^{\mathrm{e}}$, comes from the stable region $\mathbb{C}^{-}$while the other one comes from the unstable region $\mathbb{C}^{+}$. By contrast, no elements of $\widehat{H}_{\phi}^{\mathrm{o}} \circ \Gamma_{\phi}^{\mathrm{e}}$ become real. The link between $\eta_{\mathrm{bh}}^{\mathrm{e}}$ and $\eta_{c}$ depends upon the corner angle $\phi:[17$, Tab. 1]

$$
\forall \kappa \in I_{c}^{\mathrm{e}}, \eta_{\mathrm{bh}}^{\mathrm{e}}(\kappa):=+\eta_{c}>0 \quad(0<\phi<\pi), \eta_{\mathrm{bh}}^{\mathrm{e}}(\kappa):=-\eta_{c}<0 \quad(\pi<\phi<2 \pi) .
$$

As $\Gamma_{\phi}^{\mathrm{e}}(s)$ leaves $I_{c}^{\mathrm{e}}$ to enter $\mathbb{C}^{-}$, the black-hole exponent $\eta_{\mathrm{bh}}^{\mathrm{e}}$ crosses $\mathbb{R}$ towards the unstable region $\mathbb{C}^{+}$while $-\eta_{\mathrm{bh}}^{\mathrm{e}}$ crosses $\mathbb{R}$ towards the stable region $\mathbb{C}^{-}$. Eventually $\kappa_{1}$ is reached and we arrive at a second value for " $\widehat{H}_{\phi}\left(\kappa_{1}\right)$ ":

$$
\widehat{H}_{\phi} \circ \Gamma_{\phi}^{\mathrm{e}}(1)=\left\{\eta \in H_{\phi}\left(\kappa_{1}\right) \mid \mathfrak{J}(\eta)<0, \eta \neq-\eta_{\mathrm{bh}}^{\mathrm{e}}\left(\kappa_{1}\right)\right\} \cup\left\{\eta_{\mathrm{bh}}^{\mathrm{e}}\left(\kappa_{1}\right)\right\},
$$

which is shown in the bottom center plot of Figure 5 (compare with the bottom left plot).

Remark 13 (Definition of the black-hole exponent). Let us emphasize the meaning of (20). The critical exponent $\eta_{c}$ is a scalar defined in Proposition 7, where it is arbitrarily taken positive. The black-hole exponent $\eta_{\mathrm{bh}}^{\mathrm{e}}$ is defined by analytic continuation; in particular, the sign of $\eta_{\mathrm{bh}}^{\mathrm{e}}(\kappa)$ when $\kappa \in I_{c}^{\mathrm{e}}$ is not arbitrary but dependent upon the corner angle. The analyticity of $\kappa \mapsto \eta_{\mathrm{bh}}^{\mathrm{e}}(\kappa)$ follows from Proposition 11.

Crossing through $I_{c}^{\mathfrak{p}}$. Since Proposition 7 applies to both the odd and even cases, the odd-crossing path leads to a similar phenomenon. The black-hole exponent belongs to $H_{\phi}^{0}$ and has the sign (compare to (20)) [17, Tab. 1]

$$
\forall \kappa \in I_{c}^{\mathrm{D}}, \eta_{\mathrm{bh}}^{\mathrm{D}}(\kappa):=-\eta_{c}<0 \quad(0<\phi<\pi), \eta_{\mathrm{bh}}^{\mathrm{o}}(\kappa):=+\eta_{c}>0 \quad(\pi<\phi<2 \pi),
$$

which leads to a third value for " $\widehat{H}_{\phi}\left(\kappa_{1}\right)$ ":

$$
\widehat{H}_{\phi} \circ \Gamma_{\phi}^{\mathfrak{o}}(1)=\left\{\eta \in H_{\phi}\left(\kappa_{1}\right) \mid \mathfrak{J}(\eta)<0, \eta \neq-\eta_{\mathrm{bh}}^{\mathfrak{o}}\left(\kappa_{1}\right)\right\} \cup\left\{\eta_{\mathrm{bh}}^{\mathfrak{o}}\left(\kappa_{1}\right)\right\},
$$

plotted in the bottom right graph of Figure 5.

Summary. Starting from a contrast $\kappa_{0}$ in the upper half-plane we have obtained three different values for " $\widehat{H}_{\phi}\left(\kappa_{1}\right)$ ", depending on where the real axis has been crossed. To summarize, let us explicitly write down the three corresponding analytic continuations of $\widehat{H}_{\phi}$. The continuation across $\mathbb{R} \backslash \overline{I_{c}}$ is given by

$$
\widehat{H}_{\phi}(\kappa):=\widehat{H}_{\phi}^{\mathrm{e}}(\kappa) \cup \widehat{H}_{\phi}^{\mathrm{v}}(\kappa) \quad\left(\kappa \in \mathbb{C}^{+} \cup\left[\mathbb{R} \backslash \overline{I_{c}}\right] \cup \mathbb{C}^{-}\right)
$$

and the continuations across $I_{c}^{\mathrm{e}}$ and $I_{c}^{\mathrm{p}}$ are given by

$$
\widehat{H}_{\phi}^{\mid \mathfrak{e}}(\kappa):=\widehat{H}_{\phi}^{\mathrm{e} \mid \mathrm{e}}(\kappa) \cup \widehat{H}_{\phi}^{\mathrm{v} \mid \mathfrak{e}}(\kappa)\left(\kappa \in \mathbb{C}^{+} \cup I_{c}^{\mathrm{e}} \cup \mathbb{C}^{-}\right), \widehat{H}_{\phi}^{\mid \mathfrak{o}}(\kappa):=\widehat{H}_{\phi}^{\mathrm{e} \mid \mathfrak{o}}(\kappa) \cup \widehat{H}_{\phi}^{\mathrm{v} \mid \mathfrak{o}}(\kappa)\left(\kappa \in \mathbb{C}^{+} \cup I_{c}^{\mathfrak{p}} \cup \mathbb{C}^{-}\right),
$$

where

$$
\begin{aligned}
& \widehat{H}_{\phi}^{\mathfrak{e}(\mathfrak{p}) \mid \mathfrak{p}(\mathfrak{e})}(\kappa):=\widehat{H}_{\phi}^{\mathfrak{e}(\mathfrak{l})}(\kappa):=\left\{\eta \in H_{\phi}^{\mathrm{e}(\mathfrak{l})}(\kappa) \mid \mathfrak{J}(\eta)<0\right\} \\
& \left(\kappa \in \mathbb{C}^{+} \cup\left[\mathbb{R} \backslash \overline{I_{c}^{\mathrm{e}(\mathfrak{p})}}\right] \cup \mathbb{C}^{-}\right) \\
& \widehat{H}_{\phi}^{\mathfrak{e}(\mathfrak{o}) \mid \mathfrak{e}(\mathfrak{o})}(\kappa):=\left\{\eta \in H_{\phi}^{\mathfrak{e}(\mathfrak{o})}(\kappa) \mid \mathfrak{J}(\eta)<0, \eta \neq-\eta_{\mathrm{bh}}^{\mathrm{e}(\mathfrak{o})}(\kappa)\right\} \cup\left\{\eta_{\mathrm{bh}}^{\mathrm{e}(\mathfrak{o})}(\kappa)\right\} \quad\left(\kappa \in \mathbb{C}^{+} \cup I_{c}^{\mathfrak{e}(\mathfrak{o})} \cup \mathbb{C}^{-}\right) .
\end{aligned}
$$

By construction the sets $\widehat{H}_{\phi}(\kappa), \widehat{H}_{\phi}^{\mid \mathrm{e}}(\kappa)$, and $\widehat{H}_{\phi}^{\mid \mathrm{l}}(\kappa)$ are identical for $\kappa \in \mathbb{C}^{+}$but differ elsewhere. For any $\kappa \in \mathbb{C}^{-}$, $\widehat{H}_{\phi}(\kappa) \subset \mathbb{C}^{-}$while $\widehat{H}_{\phi}^{\text {le( })}(\kappa)$ contains the unstable black-hole exponent $\eta_{\mathrm{bh}}^{\mathrm{e}(\mathfrak{l})}(\kappa) \in \mathbb{C}^{+}$, see the bottom row of Figure 5.

\subsubsection{Definition of complex plasmonic resonances}

Section 2.2.2 has defined three analytic continuations to $\mathbb{C}^{-}$of the set of stable exponents (17), namely $\widehat{H}_{\phi}(\kappa)$ given by (24) and $\widehat{H}_{\phi}^{\mathrm{le}(\mathfrak{)})}(\kappa)$ given by (25). Each one is used below to either define or characterize a family of contrasts. To comment the proposed definitions, we will use the following elementary lemma.

Lemma 14. Let $\kappa \in \mathbb{C}$ and $\sigma:=\frac{1}{\kappa} \mathbb{1}_{\Omega_{m}}+\mathbb{1}_{\Omega_{d}}$. Let $u \neq 0$ be such that $(u, \kappa)$ is a solution of the PEP $(1,2,3)$ and $u \in H_{\text {loc }}^{1}\left(\mathbb{R}^{2} \backslash\left\{\boldsymbol{x}_{c}\right\}\right)$. The following properties hold: 
(a) $\int_{\mathbb{R}^{2} \backslash D} \sigma|\nabla u|^{2} \mathrm{~d} \boldsymbol{x}=-\int_{\partial D} \sigma \partial_{r} u \bar{u} \mathrm{~d} s$.

(b) If $u \in H_{\text {loc }}^{1}\left(\mathbb{R}^{2}\right)$ then $\kappa \in \mathbb{R}$.

Proof. (a) Integrate by parts $\operatorname{div}(\sigma \nabla u) \bar{u}=0$ on the annulus $\left\{R<r<R_{\infty}\right\}$ and take the limit $R_{\infty} \rightarrow \infty$ using (3) and [19, Prop. 2.75]. (b) Assume $u \in H_{\text {loc }}^{1}\left(\mathbb{R}^{2}\right)$. The regularity of $u$ on the disk $D$ enables to integrate by parts $\operatorname{div}(\sigma \nabla u) \bar{u}=0$ on $D$ to obtain $\int_{D} \sigma|\nabla u|^{2} \mathrm{~d} \boldsymbol{x}=\int_{\partial D} \sigma \partial_{r} u \bar{u} \mathrm{~d}$ s. By combining with (a) we deduce that $\int_{\mathbb{R}^{2}} \sigma|\nabla u|^{2} \mathrm{~d} \boldsymbol{x}=0$. Taking the imaginary part yields $\mathfrak{J}(\kappa)=0$.

Let us begin by defining isolated plasmonic eigenvalues.

Definition 15 (Isolated plasmonic eigenvalue). A contrast $\kappa$ is an isolated plasmonic (IP) eigenvalue if $\kappa \in(-\infty, 0] \backslash \overline{I_{c}}$ and there is a non-trivial $u \in H_{\mathrm{loc}}^{1}\left(\mathbb{R}^{2}\right)$ such that $(u, \kappa)$ solves the PEP $(1,2,3)$.

The corner asymptotic expansion of the associated eigenfunctions can be written using the continuation $\widehat{H}_{\phi}$ : for any $\eta_{\star}<0$,

$$
u(r, \theta) \underset{r \rightarrow 0}{=} c_{0}+\sum_{\rho \in\{e, 0\}} \sum_{\substack{\eta \in \widehat{H}_{\phi}^{\rho}(\kappa) \\ \eta_{\star} \mathcal{S}(\eta)}} c_{\eta}^{\rho} u_{\eta}^{\rho}(r, \theta)+O\left(r^{-\eta_{\star}}\right) \quad(r \in(0, R), \theta \in(-\pi, \pi]),
$$

where all of the local solutions $u_{\eta}^{\rho}$ in the right-hand side belong to $H^{1}(D)$, see the discussion after Proposition 5 . Isolated eigenvalues can be computed without any specific treatment of the corner. The case $\kappa \in I_{c}$ leads to the definition of embedded plasmonic eigenvalues (the odd variant is obtained by swapping " $e$ " and " $\mathrm{v}$ ").

Definition 16 (Embedded plasmonic eigenvalue). An even-critical embedded plasmonic (EP) eigenvalue is a real contrast $\kappa \in I_{c}^{\mathrm{e}}$ for which there is a non-trivial $u \in H_{\text {loc }}^{1}\left(\mathbb{R}^{2}\right)$ such that $(u, \kappa)$ solves the PEP $(1,2,3)$.

Let $u$ be an eigenfunction associated with an even-critical EP eigenvalue $\kappa \in I_{c}^{\mathrm{e}}$. The regularity of $u$ implies that its corner asymptotic expansion must only include local solutions $u_{\eta}^{\mathrm{e}}$ and $u_{\eta}^{\mathrm{o}}$ that belong to $H^{1}(D)$. For any $\eta_{\star}<0$, the expansion can be written using the continuation $\widehat{H}_{\phi}^{\text {le }}$ as

$$
u(r, \theta) \underset{r \rightarrow 0}{=} c_{0}+\sum_{\rho \in\{\mathrm{e}, \mathrm{p}\}} \sum_{\substack{\eta \in \widehat{H}_{\phi}^{\rho \mid \mathrm{e}}(\kappa) \\ \eta_{\star}<\mathcal{S}(\eta)}} c_{\eta}^{\rho} u_{\eta}^{\rho}(r, \theta)+O\left(r^{-\eta_{\star}}\right) \quad(r \in(0, R), \theta \in(-\pi, \pi]),
$$

where the singularity coefficient $c_{\eta_{\mathrm{bh}}^{\mathrm{e}}}^{\mathrm{e}}$ is null (to exclude the only local solution not in $H^{1}(D)$ in the right-hand side). Both IP and EP eigenvalues belong to the spectrum of the PEP, which can be written as a self-adjoint eigenvalue problem [1,33]. Complex resonances are intrinsic entities, obtained by continuation across the critical interval, which do not belong to the spectrum; see the definition below (the odd variant is obtained by swapping " $e$ " and " $\mathrm{D}$ ").

Definition 17 (Complex plasmonic resonance). An even-critical complex plasmonic $(C P)$ resonance is a complex contrast $\kappa \in \mathbb{C}^{-}$for which there is a non-trivial $u$ such that $(u, \kappa)$ solves the PEP $(1,2,3), u \in H_{\text {loc }}^{1}\left(\mathbb{R}^{2} \backslash\left\{\boldsymbol{x}_{c}\right\}\right)$, and $u$ admits the corner asymptotic expansion: for any $\eta_{\star}<0$,

$$
u(r, \theta) \underset{r \rightarrow 0}{=} c_{0}+\sum_{\rho \in\{\mathrm{e}, \mathrm{p}\}} \sum_{\substack{\eta \in \widehat{H}_{\phi}^{\rho \mid e}(\kappa) \\ \eta_{\star}<\mathcal{S}(\eta)}} c_{\eta}^{\rho} u_{\eta}^{\rho}(r, \theta)+O\left(r^{-\eta_{\star}}\right) \quad(r \in(0, R), \theta \in(-\pi, \pi]) .
$$

If $\kappa$ is an even-critical CP resonance then from Lemma 14(b) the associated CP resonance function $u$ cannot belong to $H_{\text {loc }}^{1}\left(\mathbb{R}^{2}\right)$, so that in (28) the singularity coefficient $c_{\eta_{\mathrm{bh}}^{\mathrm{e}}}^{\mathrm{e}}$ must be non-null. The expansion (28) implies

$$
u(r, \theta) \underset{r \rightarrow 0}{=} c_{0}+c_{\eta_{\mathrm{bh}}^{\mathrm{e}}}^{\mathrm{e}} u_{\eta_{\mathrm{bh}}^{\mathrm{e}}}^{\mathrm{e}}(r, \theta)+O\left(\left|r^{i \eta}\right|\right) \text {, with } \eta_{\mathrm{bh}}^{\mathrm{e}} \in \mathbb{C}^{+} \text {and } \eta \in \mathbb{C}^{-},
$$

where the singular behavior of $u_{\eta_{\mathrm{bh}}^{\mathrm{e}}}^{\mathrm{e}}$ is described in the discussion following Proposition 5. A comparison of (27) and (28) makes clear that even-crossing EP eigenfunctions and CP resonance functions share the same corner asymptotic expansion, the only difference being that the singularity coefficient $c_{\eta_{\mathrm{bh}}^{\mathrm{e}}}^{\mathrm{e}}$ is null for the former and non-null for the

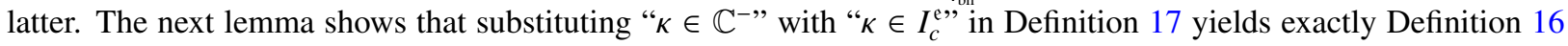
(the same result holds for the odd variant). 
Lemma 18. Let $\kappa \in \mathbb{C}$ and $u \neq 0$ be such that $(u, \kappa)$ solves the PEP (1,2,3), $u \in H_{\mathrm{loc}}^{1}\left(\mathbb{R}^{2} \backslash\left\{\boldsymbol{x}_{c}\right\}\right)$, and u satisfies (28) for any $\eta_{\star}<0$. If $\kappa \in I_{c}^{\mathrm{e}}$, then $\kappa$ is an EP eigenvalue.

Proof. We show that we must have $u \in H_{\mathrm{loc}}^{1}\left(\mathbb{R}^{2}\right)$ and $c_{\eta_{\mathrm{bh}}^{\mathrm{e}}}^{\mathrm{e}}=0$ in (28). By injecting (28) in the right-hand side of Lemma 14(a), we can conclude using [17, Eq. (28)] that the right-hand side does not depend upon the disc radius $R$. Taking the limit $R \rightarrow 0$, we conclude that $u \in H_{\text {loc }}^{1}\left(\mathbb{R}^{2}\right)$. Hence the singularity coefficient satisfies $c_{\eta_{\mathrm{bh}}^{\mathrm{e}}}^{\mathrm{e}}=0$.

Remark 19. Section 2.2 starts by picking $\kappa_{0}$ in $\mathbb{C}^{+}$; Lemma 6(b) implies that the alternative choice $\kappa_{0} \in \mathbb{C}^{-}$would lead to conjugate exponent sets and is thus equivalent. Indeed, the set of stable exponents $\widehat{H}_{\phi}^{\mathrm{e}(\mathfrak{o})}(\kappa)$ would be defined as $H_{\phi}^{\mathrm{e}(\mathfrak{D})}(\kappa) \cap \mathbb{C}^{-}$for $\kappa \in \mathbb{C}^{-}$, which is conjugate of $(17)$ since $H_{\phi}^{\mathrm{e}(\mathfrak{)})}(\bar{\kappa}) \cap \mathbb{C}^{-}=\overline{H_{\phi}^{\mathrm{e}(\mathfrak{l})}(\kappa) \cap \mathbb{C}^{-}}$. The continuations would then be carried out from $\mathbb{C}^{-}$to $\mathbb{C}^{+}$, leading to a conjugate black-hole exponent.

Remark 20. The logarithmic singularity in $r$ associated with $\eta=0$ (see Proposition 5) is absent from (28). This can be justified by noting that $0 \in H_{\phi}^{\mathrm{e}}(\kappa)$ for any $\kappa \in \mathbb{C}$. Therefore, this singularity cannot arise during the analytic continuation of a solution $u \in H_{\text {loc }}^{1}\left(\mathbb{R}^{2}\right)$ of the PEP $(1,2,3)$ with $\kappa=\kappa_{0} \in \mathbb{C}^{+}$: if this singularity was present at one contrast value then it would be present at all contrast values, contradicting the fact that $u \in H_{\mathrm{loc}}^{1}\left(\mathbb{R}^{2}\right)$.

So far, we have not discussed the existence of EP eigenvalues and CP resonances. [35, Thm. 8] shows that given a symmetric particle $\Omega_{m}$ with a $C^{2}$ boundary there exists a corner perturbation that generates EP eigenvalues. The existence of CP resonances will be investigated numerically in Section 5. In contrast with IP eigenvalues, the computation of both EP eigenvalues and CP resonances requires a particular treatment of the corner. In this work, we will use a corner complex scaling, first introduced in [17] to solve the plasmonic scattering problem with $\kappa \in I_{c}$. The applicability of this technique to our present endeavor is the subject of the next section.

\section{Definition and analysis of corner complex scaling}

This section focuses on the computational technique that will be used to compute CP resonances, namely the corner complex scaling first introduced in [17]. Section 3.1 formulates the complex-scaled PEP in Definition 21. Section 3.2 shows that the eigenvalues of the complex-scaled PEP that lie inside the so-called uncovered region are $\mathrm{CP}$ resonances or EP eigenvalues. The dependence upon the scaling parameter of the uncovered region is studied, in preparation for the numerical investigations carried out in Sections 4 and 5.

\subsection{Definition of corner complex scaling}

By definition a CP resonance $\kappa \notin \mathbb{R}$ is associated with a CP resonance function that solves the PEP $(1,2,3)$ but does not belong to $H_{\text {loc }}^{1}\left(\mathbb{R}^{2}\right)$, due to its singular behavior at the corner (29). Mathematically, the PEP can be written as a self-adjoint eigenvalue problem $[1,33]$ so its spectrum must be real-valued. Numerically, a standard Lagrange FE discretization of the PEP yields a Hermitian generalized eigenvalue problem. The general principle of complex scaling is to define a non self-adjoint complex-scaled PEP that relies on a scaling parameter $\alpha \in \mathbb{C}$, which we denote $\operatorname{PEP} \alpha$, such that some eigenvalues of the PEP $\alpha$ turn out to be CP resonances of the original PEP.

The origin of complex scaling is typically traced back to [45] and [46], where it is used to compute scattering resonances of the Hamiltonian operator [47, Chap. 16]. In these works, the complex-scaling method can be understood as consisting in surrounding the scatterer by an absorbing layer that does not induce spurious reflections: as such, it is similar to the PML method, first proposed on physical grounds in [48] for electromagnetic waves in the time domain. The link between the PML and complex-scaling methods has been highlighted early, see [49] and [50] where "coordinate stretching" and "coordinate mapping" methods are discussed in the context of the PML method. For an analysis of the computation of scattering resonances using a PML, see [51] and [52, §2.7]. Applications in fluid mechanics and acoustics can be found in e.g. [53] and [54]. In the remainder of this paper we will rely on a variant of complex scaling where the scaling is applied not at infinity, as in the works quoted above, but at the corner of the particle $\Omega_{m}$. This scaling has been introduced in [17], where it is used to solve the plasmonic scattering problem with a critical contrast (i.e. $k \in I_{c}$ ).

Since CP resonance functions are singular solely at the corner, the PEP and the PEP $\alpha$ need only differ in a neighborhood of the corner. Let us consider the neighborhood $D$ depicted in Figure 1b, where $(1,2)$ reduces to (9). 
The intuition behind the definition of the complex-scaled eigenproblem is as follows. Let $\kappa$ be a CP resonance. By definition, it is associated with a CP resonance function that does not belong to $H_{\text {loc }}^{1}\left(\mathbb{R}^{2}\right)$ since from (29)

$$
u(r, \theta) \underset{r \rightarrow 0}{\sim} c_{0}+c_{\eta}^{\rho} \Phi_{\eta}^{\rho}(\theta) r^{i \eta}
$$

for some $\eta \in \mathbb{C}^{+}$and $\rho \in\{\mathfrak{e}, \mathfrak{D}\}$. We would like $\kappa$ to be associated with an eigenfunction of the PEP $\alpha$ that satisfies

$$
u_{\alpha}(r, \theta) \underset{r \rightarrow 0}{=} c_{0}+O\left(r^{i \frac{\eta}{\alpha}}\right),
$$

where $\alpha$ is a scaling parameter chosen so that $u_{\alpha} \in H_{\mathrm{loc}}^{1}\left(\mathbb{R}^{2}\right)$, i.e. $\mathfrak{J}(\eta / \alpha)<0$. This can be achieved through the substitution " $r \partial_{r} \rightarrow \alpha r \partial_{r}$ " in (9), which leads to the following definition of the PEP $\alpha$, where $\Delta_{\alpha}$ denotes the radiallyscaled Laplacian:

$$
\Delta_{\alpha} u(r, \theta):=\frac{1}{r^{2}} \alpha r \partial_{r}\left(\alpha r \partial_{r} u\right)(r, \theta)+\frac{1}{r^{2}} \partial_{\theta}\left(\partial_{\theta} u\right)(r, \theta) .
$$

Definition 21 (Complex-scaled PEP). Let $\alpha \in \mathbb{C} \backslash\{0\}$ and $D_{\alpha}:=\left\{\left|\boldsymbol{x}-\boldsymbol{x}_{c}\right|<R_{\alpha}\right\}$ with $R_{\alpha} \leq R$, as depicted in Figure 6. The complex-scaled PEP, denoted PEP $\alpha$, is: find $\left(u_{\alpha}, \kappa\right)$ such that

$$
\Delta u_{\alpha}(\boldsymbol{x})=0 \quad\left(\boldsymbol{x} \in\left(\Omega_{m} \cup \Omega_{d}\right) \backslash \overline{D_{\alpha}}\right), \Delta_{\alpha} u_{\alpha}(\boldsymbol{x})=0 \quad\left(\boldsymbol{x} \in\left(\Omega_{m} \cup \Omega_{d}\right) \cap D_{\alpha}\right),
$$

with the transmission conditions

$$
\begin{array}{ll}
u_{\alpha \mid \Omega_{m}}(\boldsymbol{x})=u_{\alpha \mid \Omega_{d}}(\boldsymbol{x}), \partial_{n} u_{\alpha \mid \Omega_{m}}(\boldsymbol{x})=\kappa \partial_{n} u_{\alpha \mid \Omega_{d}}(\boldsymbol{x}) \quad\left(\boldsymbol{x} \in \partial \Omega_{m}\right) \\
u_{\alpha \mid D_{\alpha}}(\boldsymbol{x})=u_{\alpha|\Omega| \overline{D_{\alpha}}}(\boldsymbol{x}), \alpha \partial_{n} u_{\alpha \mid D_{\alpha}}(\boldsymbol{x})=\partial_{n} u_{\alpha|\Omega| \overline{D_{\alpha}}}(\boldsymbol{x}) & \left(\boldsymbol{x} \in \partial D_{\alpha}\right),
\end{array}
$$

and the decay condition at infinity $u_{\alpha}(x) \underset{|x| \rightarrow \infty}{=} O\left(|x|^{-1}\right)$.

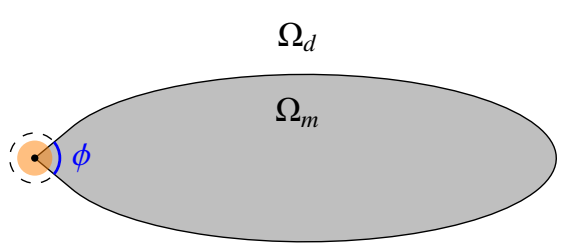

(a) Overview with corner neighborhood highlighted.

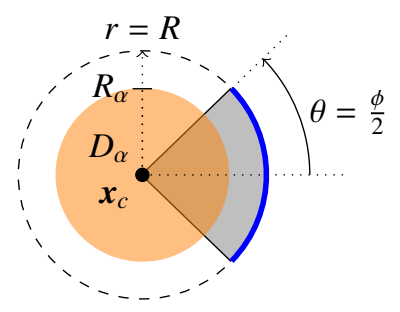

(b) Corner neighborhoods $D$ and $D_{\alpha}$.

Fig. 6: Particle $\Omega_{m}$ with a complex-scaling region $D_{\alpha} \subset D$ around the corner.

Since the scaling does not affect the orthoradial part of the Laplacian, the PEP and the PEP $\alpha$ share the same corner dispersion relations (7). This implies that Proposition 5 also holds for the PEP $\alpha$ provided that " $r$ " " is substituted by " $r$ in/s". We introduce the critical curves

$$
I_{c}^{\mathrm{e}(\mathfrak{o}), \alpha}:=\left\{\kappa \in \mathbb{C} \mid \exists \eta \in H_{\phi}^{\mathrm{e}(\mathfrak{o})}(\kappa) \backslash\{0\}: \frac{\eta}{\alpha} \in \mathbb{R}\right\}, I_{c}^{\alpha}:=I_{c}^{\mathrm{e}, \alpha} \cup I_{c}^{\mathfrak{p}, \alpha},
$$

which when $\alpha \in \mathbb{R}^{*}$ coincide with the critical intervals $I_{c}^{\mathrm{e}(\mathfrak{o})}$ and $I_{c}$ respectively. An isolated eigenvalue $\kappa \notin \overline{I_{c}^{\alpha}}$ of the $\operatorname{PEP} \alpha$ is associated with an eigenfunction that admits the local expansion

$$
u_{\alpha}(r, \theta) \underset{r \rightarrow 0}{=} c_{0}+\sum_{\substack{\eta \in \widehat{H}_{\phi}^{\mathrm{e}, \alpha}(\kappa) \\ \eta_{\star}<\mathcal{S}\left(\frac{\eta}{\alpha}\right)}} c_{\eta}^{\mathrm{e}} r^{i \frac{\eta}{\alpha}} \Phi_{\eta}^{\mathrm{e}}(\theta)+\sum_{\substack{\eta \in \widehat{H}_{\phi}^{\mathrm{0}, \alpha}(\kappa) \\ \eta_{\star}<\mathcal{S}\left(\frac{\eta}{\alpha}\right)}} c_{\eta}^{\mathrm{o}} r^{i \frac{\eta}{\alpha}} \Phi_{\eta}^{\mathfrak{0}}(\theta)+O\left(r^{-\eta_{\star}}\right) \quad\left(r \in\left(0, R_{\alpha}\right), \theta \in(-\pi, \pi]\right)
$$

for any $\eta_{\star}<0$, where

$$
\widehat{H}_{\phi}^{\mathrm{e}(\mathfrak{o}), \alpha}(\kappa):=\left\{\eta \in H_{\phi}^{\mathrm{e}(\mathfrak{o})}(\kappa) \mid \mathfrak{J}(\eta / \alpha)<0\right\}, \widehat{H}_{\phi}^{\alpha}(\kappa):=\widehat{H}_{\phi}^{\mathrm{e}, \alpha}(\kappa) \cup \widehat{H}_{\phi}^{\mathrm{v}, \alpha}(\kappa)
$$

are the sets of stable exponents that we associate with the $\operatorname{PEP} \alpha$, which reduce to $\widehat{H}_{\phi}^{\text {e( ) }}(\kappa)$ and $\widehat{H}_{\phi}(\kappa)$ when $\alpha>0$. The next section explains how the PEP $\alpha$ can be used to compute CP resonances. 

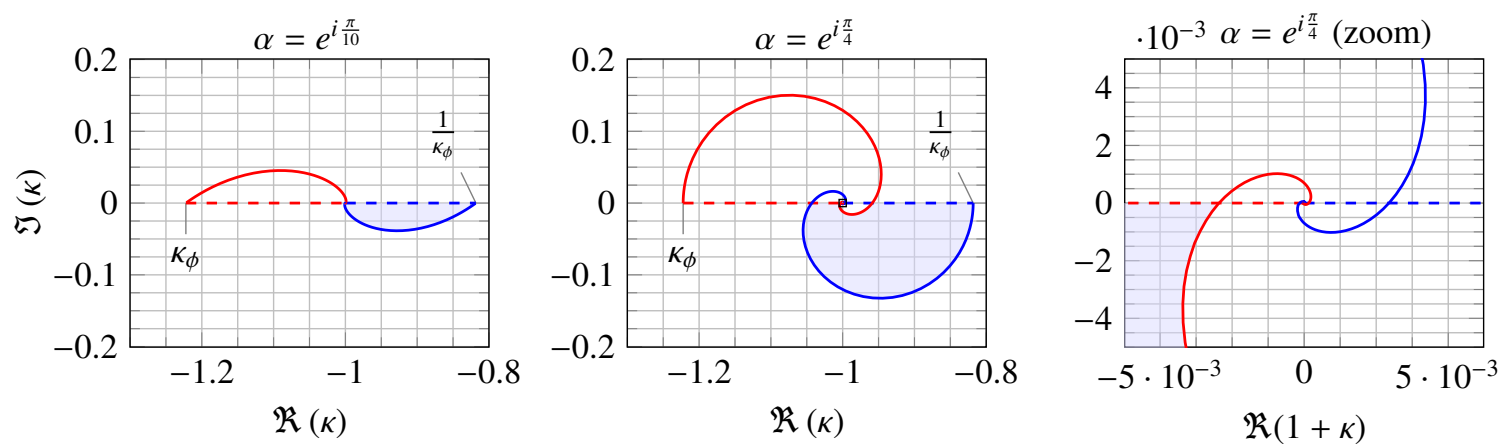

Fig. 7: Even uncovered region $U_{\phi}^{\mathrm{e}, \alpha}$ for $\phi=0.9 \pi$ and two values of $\arg (\alpha)$ in $[0, \pi / 2]$. (一): even critical curve $I_{c}^{\mathrm{e}, \alpha},(-): I_{c}^{\mathrm{v}, \alpha}$.

\subsection{Computation of complex plasmonic resonances}

Assume $\kappa \notin \mathbb{R}$ is an isolated eigenvalue of the PEP $\alpha$. For $\kappa$ to be an even-critical (resp. odd-critical) CP resonance, the scaling parameter $\alpha$ must be chosen so that

$$
\widehat{H}_{\phi}^{\alpha}(\kappa)=\widehat{H}_{\phi}^{\mid \mathrm{e}(\mathfrak{p})}(\kappa),
$$

i.e. the set of exponents that are stable after a rotation of angle $-\arg (\alpha)$ in the $\eta$-plane is exactly $\widehat{H}_{\phi}^{\text {le(b) }}(\kappa)$. The condition (33) can be read as a constraint on $\alpha$ given $\kappa$. However in practice $\alpha$ is an input parameter so it is also useful to consider it as a constraint on $\kappa$ given $\alpha$ : it effectively delimits a region of the complex plane given by

$$
K_{\phi}^{\mathrm{e}(\mathfrak{D}), \alpha}:=\{\kappa \in \mathbb{C} \mid \text { Stability condition (33) holds }\},
$$

which is where computable $\mathrm{CP}$ resonances lie. The link between isolated eigenvalues of the $\operatorname{PEP} \alpha$ and $\mathrm{CP}$ resonances is given in the next proposition (the odd variant is obtained by replacing " $\mathrm{e}$ " by " $\mathrm{v}$ ").

Proposition 22. Let $\alpha \in \mathbb{C} \backslash \mathbb{R}$. Let $\left(u_{\alpha}, \kappa\right)$ be a solution of the $\operatorname{PEP} \alpha$ such that $\kappa$ belongs to the even uncovered region

$$
U_{\phi}^{\mathrm{e}, \alpha}:=K_{\phi}^{\mathrm{e}, \alpha} \cap \overline{\mathbb{C}^{-}} .
$$

If $\mathfrak{J}(\kappa)<0($ resp. $\mathfrak{J}(\kappa)=0)$ then $\kappa$ is an even-critical CP resonance (resp. EP eigenvalue) and the function defined by

$$
u(\boldsymbol{x}):=u_{\alpha}(\boldsymbol{x}) \quad\left(\boldsymbol{x} \in\left(\Omega_{m} \cup \Omega_{d}\right) \backslash \overline{D_{\alpha}}\right), u(r, \theta):=u_{\alpha}\left(r^{\alpha}, \theta\right) \quad\left(r \in\left(0, R_{\alpha}\right), \theta \in(-\pi, \pi]\right)
$$

is its associated CP resonance function (resp. eigenfunction).

Proof. Since $\kappa$ is an isolated eigenvalue of the PEP $\alpha, u_{\alpha}$ admits the expansion (32) at the corner. The substitution $r \rightarrow r^{\alpha}$ in (32) yields the expansion of $u$ at the corner (this is not a change of variable but an analytic continuation of $u_{\alpha}(\cdot, \theta)$ from $\left(0, R_{\alpha}\right) \subset \mathbb{R}$ to $\left(0, R_{\alpha}\right)^{\alpha} \subset \mathbb{C}$, which we do not further justify here). $u$ solves the PEP $(1,2,3)$ and satisfies

$$
u(r, \theta) \underset{r \rightarrow 0}{=} c_{0}+\sum_{\substack{\eta \in \widehat{H}_{\phi}^{\mathrm{e}, \alpha}(\kappa) \\ \eta_{\star}<\mathcal{S}(\eta)}} c_{\eta}^{\mathrm{e}} r^{i \eta} \Phi_{\eta}^{\mathrm{e}}(\theta)+\sum_{\substack{\eta \in \widehat{H}_{\phi}^{\mathrm{o}, \alpha}(\kappa) \\ \eta_{\star}<\mathcal{S}(\eta)}} c_{\eta}^{\mathfrak{o}} r^{i \eta} \Phi_{\eta}^{\mathrm{j}}(\theta)+O\left(r^{-\eta_{\star}}\right) \quad(r \in(0, R), \theta \in(-\pi, \pi])
$$

for any $\eta_{\star}<0$. Since $\kappa \in K_{\phi}^{\mathrm{e}, \alpha}$, we can use the stability condition (33) to conclude that $\kappa$ is either an even-critical EP eigenvalue or a $\mathrm{CP}$ resonance.

If $\alpha \in \mathbb{R}^{*}, U_{\phi}^{\mathfrak{e}(\mathfrak{D}), \alpha}$ is empty since $K_{\phi}^{\mathfrak{e}(\mathfrak{D}), \alpha}=\mathbb{C}^{+}$. From the above result, the usefulness of the PEP $\alpha$ relies on the ability to choose a non-real $\alpha$ so that the uncovered region includes the part of the complex plane where CP resonances are sought.

Let us now discuss how to compute the uncovered region, which is of practical importance to help choose the scaling parameter $\alpha$. A straightforward method is to sample the complex plane (in $\kappa$ ) and evaluate (33). However this is costly since it requires the accurate computation of the exponents set $H_{\phi}(\kappa)$, which can be challenging depending 
on the value of $\phi$. A more accurate alternative is to compute the boundaries of the uncovered region, which reduces to computing the critical curves (31). Indeed, we have the identities

$$
U_{\phi}^{\mathfrak{e}(\mathfrak{D}), \alpha}:=\left\{\kappa \in \overline{\mathbb{C}^{-}} \mid \widehat{H}_{\phi}^{\alpha}(\kappa)=\widehat{H}_{\phi}^{\mid \mathfrak{e}(\mathfrak{l})}(\kappa)\right\}=\left\{\kappa \in \overline{\mathbb{C}^{-}} \mid \widehat{H}_{\phi}^{\alpha}(\kappa) \supset \widehat{H}_{\phi}^{\mathrm{le}(\mathfrak{l})}(\kappa)\right\}=\left\{\kappa \in \overline{\mathbb{C}^{-}} \mid \forall \eta \in \widehat{H}_{\phi}^{\mathrm{le}(\mathfrak{l})}(\kappa), \mathfrak{J}(\eta / \alpha)<0\right\},
$$

so that the boundary satisfies

$$
\partial U_{\phi}^{\mathrm{e}(\mathfrak{D}), \alpha}=\left\{\kappa \in \overline{\mathbb{C}^{-}} \mid \exists \eta \in \widehat{H}_{\phi}^{\mid \mathrm{e}(\mathfrak{o})}(\kappa), \mathfrak{J}(\eta / \alpha)=0\right\} \subset\left\{\kappa \in \overline{\mathbb{C}^{-}} \mid \exists \eta \in H_{\phi}(\kappa) \backslash\{0\}, \mathfrak{I}(\eta / \alpha)=0\right\} \subset \overline{I_{c}^{\alpha}} \cap \overline{\mathbb{C}^{-}} .
$$

The interest of this inclusion is that plotting the critical curves is straightforward since they admit the parametric representations

$$
I_{c}^{\mathfrak{e}(\mathfrak{D}), \alpha}=\left\{\kappa \in \mathbb{C} \mid \exists \tilde{\eta}>0: f_{\phi}^{\mathfrak{e}(\mathfrak{D})}(\alpha \tilde{\eta}, \kappa)=0\right\}=\left\{\frac{\psi_{\phi}(\alpha \tilde{\eta})_{(++)}^{-} 1}{\psi_{\phi}(\alpha \tilde{\eta})_{(-)}^{+} 1} \mid \tilde{\eta} \in(0, \infty)\right\},
$$

where $\psi_{\phi}$ is given by (11). These parametric representations show that both intervals are even with respect to $\alpha$ and only depend upon $\arg (\alpha)$, so that from now on we always assume

$$
|\alpha|=1, \arg (\alpha) \in\left[-\frac{\pi}{2}, \frac{\pi}{2}\right] .
$$

Figure 7 plots the even uncovered region for $\phi=0.9 \pi$ and two scaling parameter values. (The cases $\phi \in(0, \pi)$ and $\phi \in(\pi, 2 \pi)$ are similar, with even and odd switching roles.) When $\alpha \neq 1$, the two critical curves spiral around the branch point $\kappa=-1$, which reflects its logarithmic nature. The plots also highlight the impact of $\arg (\alpha)$ on the uncovered region. The closer $\arg (\alpha)$ is to $\pi / 2$, the further away from -1 the even uncovered region is, due to the odd critical curve $I_{c}^{\mathrm{p}, \alpha}$. This shows that there is a trade-off between the area of the uncovered region and how close it gets to -1 . Since plasmonic eigenvalues accumulate at -1 , practical computations will typically favor small values of $\arg (\alpha)$.

Remark 23. As already stated in Remark 19, starting from $\kappa_{0} \in \mathbb{C}^{-}$would conjugate everything. In particular, the uncovered region would be included in $\overline{\mathbb{C}^{+}}$.

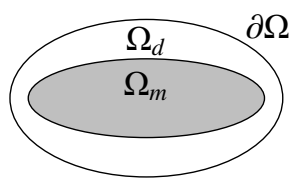

(a) Cartesian coordinates $(x, y)$.

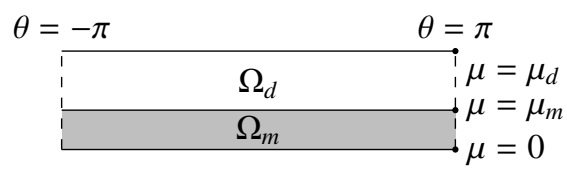

(b) Elliptic coordinates $(\mu, \theta)$ defined by (D.2).

Fig. 8: Elliptical particle in a bounded dielectric domain $\Omega_{d}$. The boundaries $\partial \Omega_{m}$ and $\partial \Omega$ are non-circular confocal ellipses.
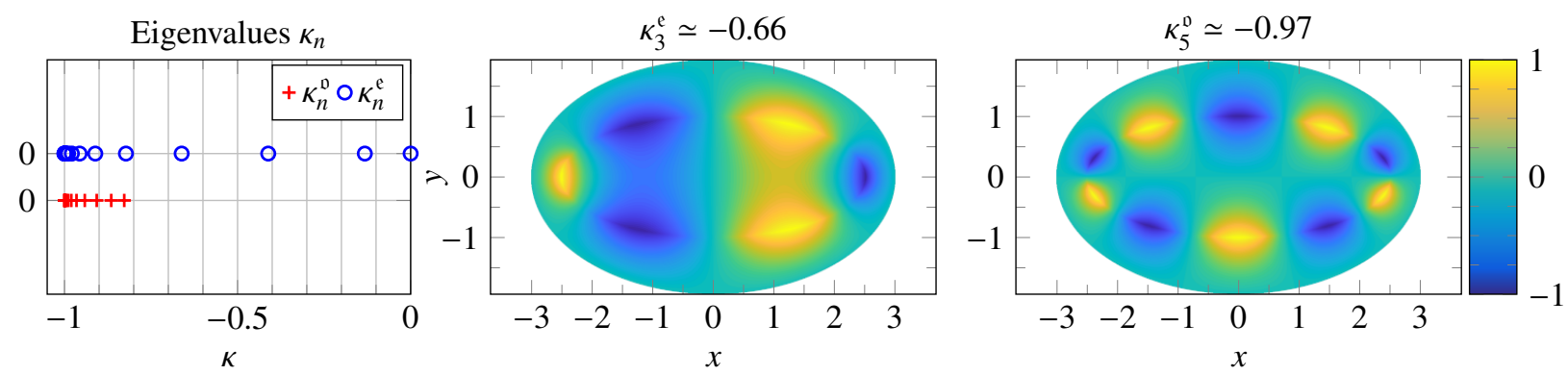

Fig. 9: Eigenvalues (D.1) and eigenfunctions $u /\|u\|_{\infty}$ of the PEP (36) for an elliptical particle (see Figure 8a). The sign-changing interface $\partial \Omega_{m}$ has semi-axes $a_{m}=2.5$ and $b_{m}=1$, and the outer boundary $\partial \Omega$ is a confocal ellipse such that $a_{d}=3$. 


\section{Validation of the finite element discretization}

The purpose of this section is to validate the FE discretization of the PEP $\alpha$ that will be used in Section 5 to compute $\mathrm{CP}$ resonances. The validation is carried out on two subproblems. Section 4.1 considers an elliptical particle to validate the computation of IP eigenvalues. It highlights that spectral accuracy is strongly controlled by a local mesh symmetry with respect to the sign-changing interface. Section 4.2 considers a corner geometry to validate the discretization of the critical interval, with and without complex scaling.

\subsection{Discretization of isolated plasmonic eigenvalues}

To validate the discretization of IP eigenvalues, we take

$$
\Omega_{m}:=\left\{(x, y) \in \mathbb{R}^{2} \mid\left(x / a_{m}\right)^{2}+\left(y / b_{m}\right)^{2}<1\right\}, \Omega:=\left\{(x, y) \in \mathbb{R}^{2} \mid\left(x / a_{d}\right)^{2}+\left(y / b_{d}\right)^{2}<1\right\}, \Omega_{d}:=\Omega \backslash \overline{\Omega_{m}},
$$

with $a_{m}=2.5, b_{m}=1, a_{d}=3$, and $b_{d}=\sqrt{a_{d}^{2}-c^{2}}$ with $c=\sqrt{a_{m}^{2}-b_{m}^{2}}$, see Figure 8a. For simplicity we impose a Dirichlet boundary condition on $\partial \Omega$, which excludes the trivial solutions $(u=\mathrm{cst}, \kappa)$. The weak formulation of the PEP is then: find $(u, \kappa) \in H_{0}^{1}(\Omega) \times \mathbb{C}$ such that for all $v \in H_{0}^{1}(\Omega)$,

$$
a_{\Omega_{m}}^{(x, y)}(u, v)=-\kappa a_{\Omega_{d}}^{(x, y)}(u, v), \text { where } a_{X}^{(x, y)}(u, v):=\int_{X} \nabla u(\boldsymbol{x}) \cdot \nabla v(\boldsymbol{x}) \mathrm{d} \boldsymbol{x} .
$$

Since $\partial \Omega_{m}$ is smooth, the point spectrum is made of a sequence of isolated eigenvalues $\kappa_{n}$ that accumulate at $\kappa=-1$ [1, Thm. 1] [33, Thm. A]. Since $\partial \Omega_{m}$ and $\partial \Omega$ are non-circular confocal ellipses, Laplace's equation (1) is separable in elliptic coordinates and the eigenvalues $\kappa_{n}^{\mathrm{e}(\mathrm{o})}$ are given by (D.1); the corresponding eigenfunctions are even (resp. odd) with respect to the major axis, see Figure 9. The closer $\kappa_{n}^{\mathrm{e}(\mathfrak{l})}$ is to -1 , the more oscillating the eigenfunction.

Remark 24. The distinction between $\kappa_{n}^{\mathrm{e}}$ and $\kappa_{n}^{\mathfrak{0}}$ is only made in preparation for Section 5.

The PEP (36) can be readily discretized using Lagrange finite elements [55]: after eliminating the DoF on $\partial \Omega$, this leads to the $N_{h}$-dimensional generalized eigenvalue problem

$$
A_{\Omega_{m}}^{(x, y)} U=-\kappa A_{\Omega_{d}}^{(x, y)} U,
$$

where $A_{\Omega_{m}}^{(x, y)}$ and $A_{\Omega_{d}}^{(x, y)}$ are both real symmetric and positive (but not definite) matrices. In this work, we use the weak form PDE interface of COMSOL 5.4: the FE matrices are obtained using a mix of $\mathbb{P}_{2}$ and $\mathbb{Q}_{2}$ isoparametric Lagrange elements [56], while the generalized eigenvalue problem is solved using the implicitly restarted Arnoldi method with shift-and-invert from ARPACK [57] with a tolerance of $10^{-6}$. To compute a spectrum that spans a large region of $\mathbb{C}$, the eigenproblem is solved several times for various values of the shift $\kappa_{0}$ (which is always taken non-null, since $A_{\Omega_{m}}^{(x, y)}$ is singular).
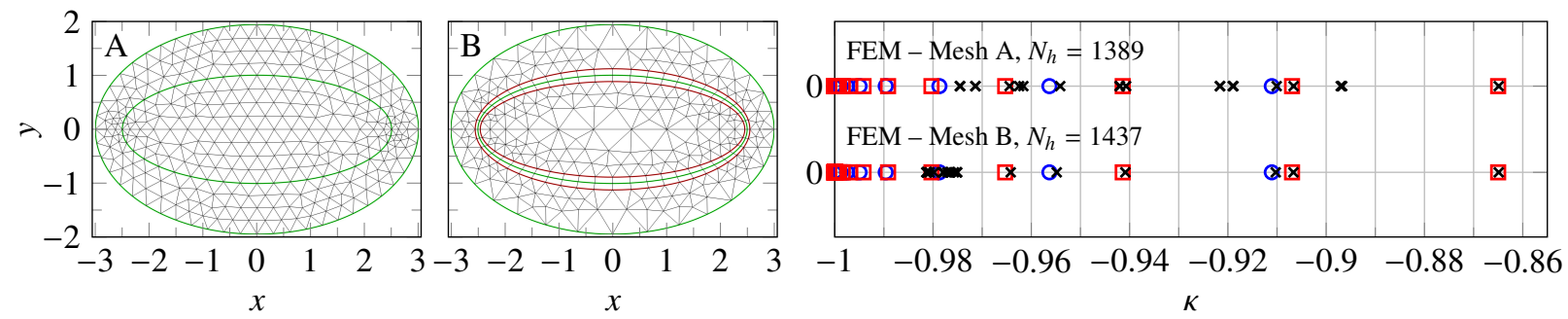

Fig. 10: Solution of the PEP (36). (Left) Mesh A: 616 elements, unstructured. (Center) Mesh B: 574 elements, structured region around $\partial \Omega_{m}$ built using two ellipses defined though (38) and highlighted in red. (Right) $(\mathbf{\times})$ : computed, $(\bigcirc)$ : exact even $\kappa_{n}^{\mathrm{e}}$, $(\square)$ : exact odd $\kappa_{n}^{\mathrm{o}}$. 

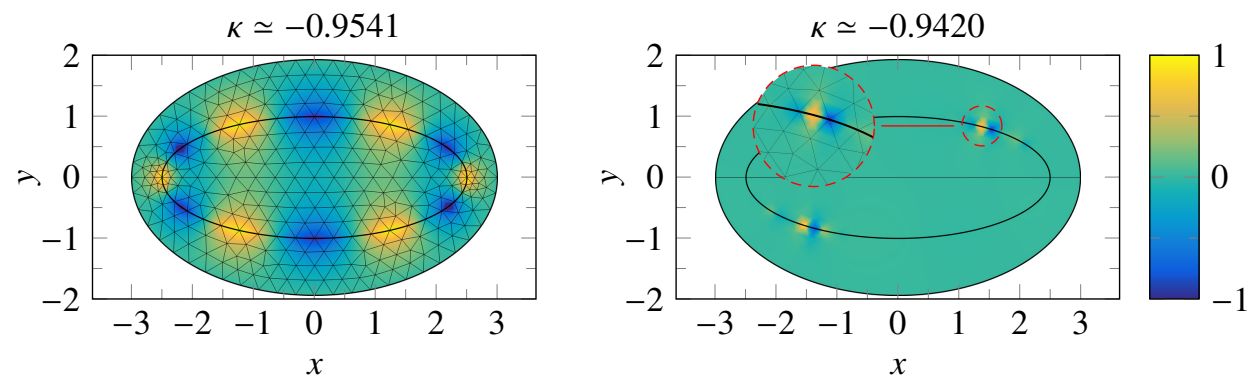

Fig. 11: Eigenfunctions $u /\|u\|_{\infty}$ computed using the mesh A shown in Figure 10. (Left) Correct eigenfunction (surface plasmon), oscillating over the whole interface. (Right) Spurious eigenfunction (spurious plasmon), localized at only a few nodes of the interface.

\section{Meshing strategy}

Let us first consider a triangular mesh symmetric with respect to the $x$-axis, as depicted in the left graph of Figure 10. The computed spectrum shown in the right plot exhibits significant pollution, with the largest spurious eigenvalue at $\kappa_{\mathrm{sp}} \simeq-0.895$. Figure 11 plots one of the spurious plasmons: in contrast with surface plasmons, which oscillate over the whole interface $\partial \Omega_{m}$, spurious plasmons are localized at only a few nodes of the interface. This spectral pollution is not caused by a resolution problem, as the mesh used in this example is fine enough to resolve plasmons much closer to -1 than $\kappa_{\mathrm{sp}}$. In practice, this means that refining the mesh is not an effective strategy to deal with these spurious plasmons, i.e. it does not systematically move the spurious eigenvalues closer to -1 . Typically, mesh refinement even spreads spurious eigenvalues and increases $\kappa_{\mathrm{sp}}$.

An effective strategy to reduce this spectral pollution is to employ a mesh with a structured quad layer at the signchanging interface $\partial \Omega_{m}$, as depicted in the center graph of Figure 10. The mesh is constructed using two additional confocal ellipses highlighted in red. Crucially, these two ellipses must be chosen so that the structured layer is (approximately) symmetric in elliptic coordinates: first, the semi-axis $a_{o}$ of the outer ellipse is chosen in $\left(a_{m}, a_{d}\right)$; then, the inner ellipse is obtained from

$$
a_{i}=c \cosh \left[2 \mu_{m}-\mu_{o}\right]=c \cosh \left[2 \operatorname{acosh}\left(\frac{a_{m}}{c}\right)-\operatorname{acosh}\left(\frac{a_{o}}{c}\right)\right],
$$

where $c$ is the focal distance of $\partial \Omega_{m}$. The use of isoparametric elements enables to better approximate the inner and outer ellipses, thus improving the symmetry of the structured layer. In addition, the orthoradial element distribution along $\partial \Omega_{m}$ is refined in the high-curvature region using an ad hoc geometric distribution.

The right plot of Figure 10 shows that spurious eigenvalues have been moved significantly closer to -1 , although the number of DoF is similar. Using triangles instead of quads in the structured layer would significantly worsen the results (as this would degrade the quality of the symmetry in elliptic coordinates): the best results are obtained with either mixed quad/tri meshes (as used in this work) or full quad meshes.

This meshing strategy is inspired by the works $[58,59]$, where the interest of mesh symmetry to discretize signchanging problems is highlighted and investigated. Based on this result, from now on, all the meshes considered will be structured and (at least approximately) symmetric at the sign-changing interface.
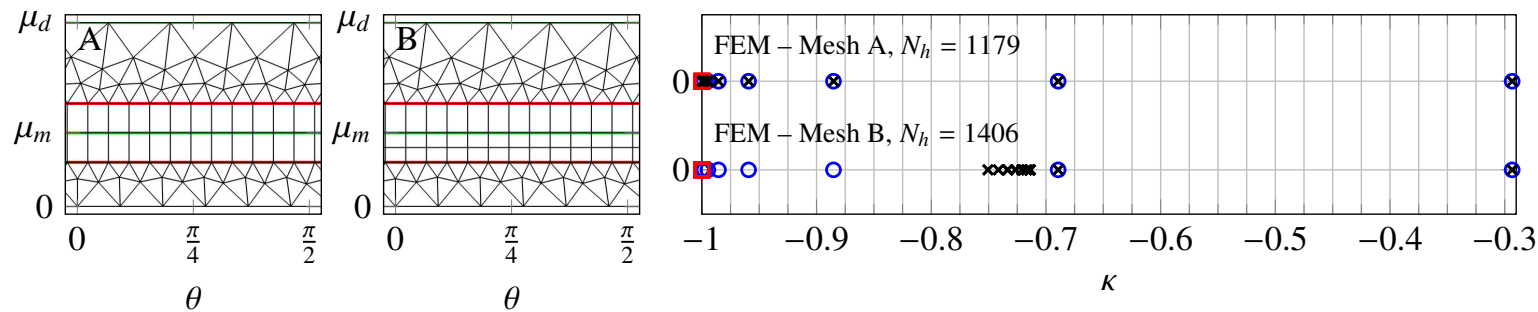

Fig. 12: Solution of the PEP (36) in elliptic coordinates. (Left) Mesh A: 459 elements, symmetric structured layer at the sign-changing interface. (Center) Mesh B: 504 elements, identical to Mesh A except that the symmetry of the structured layer has been broken by sub-dividing one side. (Right) (x): computed, $(\bigcirc)$ : exact even $\kappa_{n}^{\mathrm{e}},(\square)$ : exact odd $\kappa_{n}^{\mathrm{D}}$. 

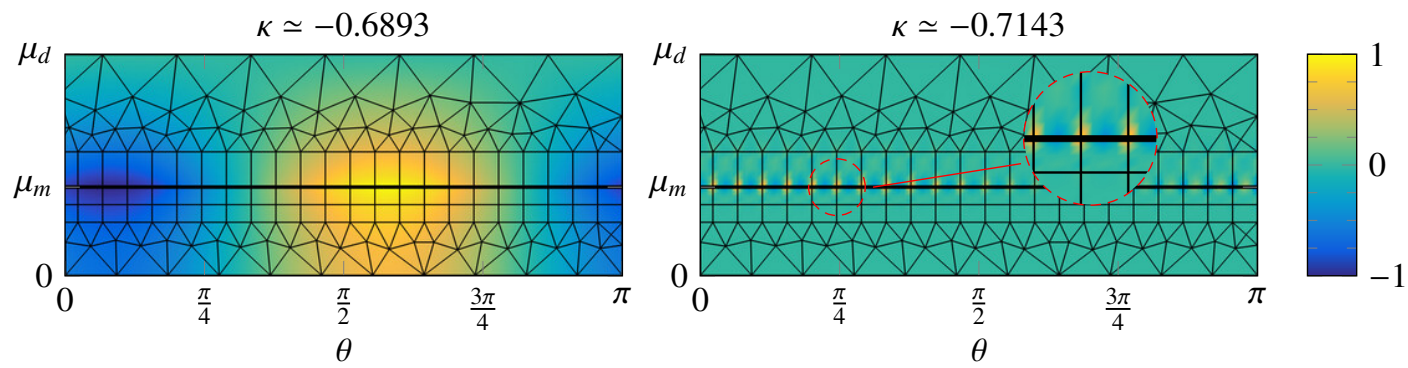

Fig. 13: Eigenfunctions $u /\|u\|_{\infty}$ using mesh B from Figure $12\left(\mu_{m}=0.5, \mu_{d}=1.25\right)$. (Left) Surface plasmon. (Right) Spurious plasmon.

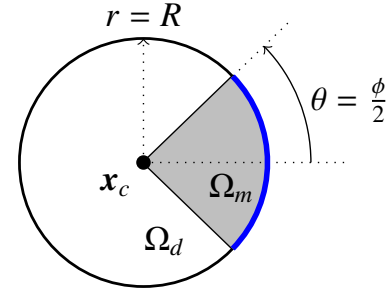

(a) Without complex-scaling region.

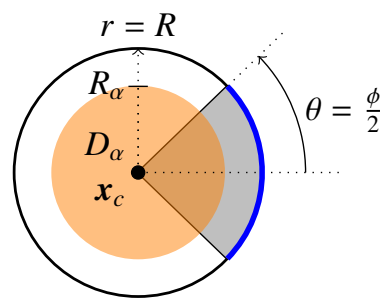

(b) With complex-scaling region $D_{\alpha} \subset D$.

Fig. 14: Corner geometry used to validate the discretization of the critical interval.

\section{Justification of the meshing strategy}

The meshing strategy proposed above relies on (38) to define the structured layer, which can be justified using the following numerical experiment. Consider the elliptic coordinate system (D.2) associated with $\partial \Omega_{m}$ (see Figure 8b): the weak formulation is (36) with $\mathrm{d} x=\mathrm{d} \mu \mathrm{d} \theta, \nabla u=\left[\partial_{\mu} u, \partial_{\theta} u\right]$, and periodic boundary conditions. The computed spectra plotted in Figure 12 show that even a small deviation from symmetry with respect to $\left\{\mu=\mu_{m}\right\}$ in the structured layer induces spectral pollution. Figure 13 compares a surface and a spurious plasmon to highlight that the pattern already observed in Cartesian coordinates can be reproduced in elliptic coordinates.

Remark 25. The eigenproblem (37) is sparse and $N_{h}$-dimensional. Using static condensation, it can be rewritten as a dense and $N_{i}$-dimensional eigenproblem, where $N_{i}$ is the number of DoF on $\partial \Omega_{m}$. Based on our numerical experiments (not shown here), static condensation yields no accuracy gain and in particular suffers from the same pollution issue. By contrast, even a crude BE discretization does not suffer from this pollution effect. Intuitively, this confirms that the pollution seen with FE comes from the discretization of the normal direction.

\subsection{Discretization of the critical interval}

To validate the discretization of $I_{c}$ and $I_{c}^{\alpha}$ we consider the corner geometry (see Figure 14a):

$$
\Omega_{m}:=\left\{(x, y) \in \mathbb{R}^{2}|| x+i y|<R,| \arg (x+i y) \mid<\phi / 2\right\}, \Omega:=\left\{(x, y) \in \mathbb{R}^{2}|| x+i y \mid<R\right\}, \Omega_{d}:=\Omega \mid \overline{\Omega_{m}} .
$$

\section{Corner without complex scaling}

Let us first consider the corner without complex scaling, for which the spectrum of the PEP (36) reduces to the critical interval (10). A natural approach is to mesh in Cartesian coordinates; for a right-angle corner, this can lead to considering a mesh like the one depicted in the left plot of Figure 15, where there is a structured layer around the sign-changing interface. However, the right graph shows that even with a fairly small element size, the critical interval is not satisfactorily resolved.

A first alternative is to mesh in polar coordinates: find $(u, \kappa) \in H_{p}^{1} \times \mathbb{C}$ such that for all $v \in H_{p}^{1}$,

$$
\int_{\Theta_{m}} \int_{R_{\mathrm{TR}}}^{R} \nabla u \cdot \nabla v r \mathrm{~d} r \mathrm{~d} \theta=-\kappa \int_{\Theta_{d}} \int_{R_{\mathrm{TR}}}^{R} \nabla u \cdot \nabla v r \mathrm{~d} r \mathrm{~d} \theta,
$$



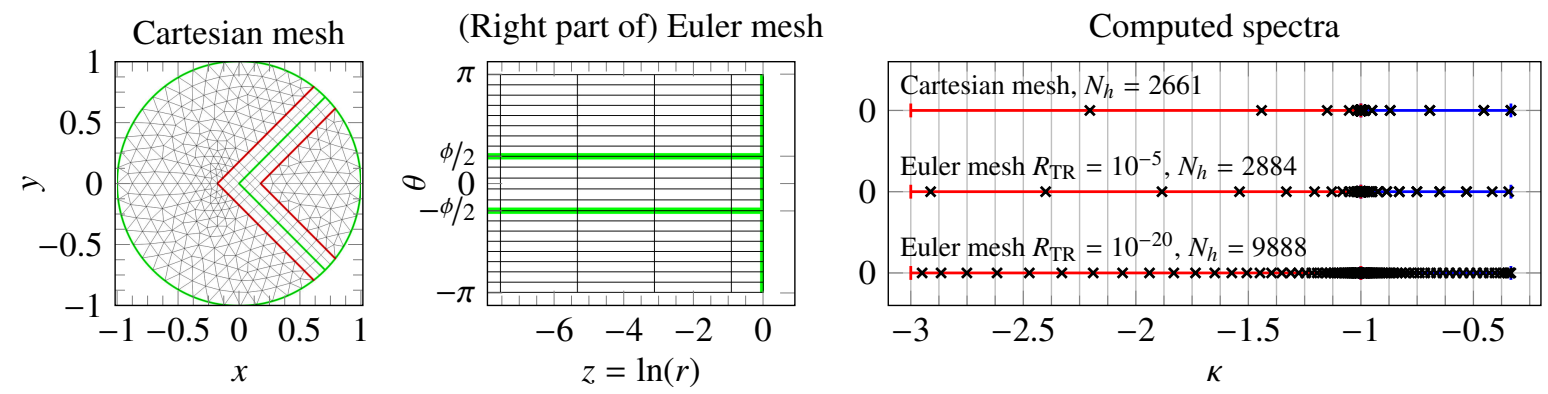

Fig. 15: Comparison between the Cartesian (36) and Euler (40) formulations of the PEP for the corner geometry depicted in Figure 14a with $\phi=\pi / 2$ and $R=1$. (Left) Example of Cartesian mesh. (Center) Example of Euler mesh. (Right) (×): computed, (一): exact even $I_{c}^{\mathrm{e}}$, (一): exact odd $I_{c}^{\mathfrak{D}}$. The two Euler meshes have the same element size.

where

$$
H_{p}^{1}:=\left\{u \in H^{1}\left(\left(R_{\mathrm{TR}}, R\right) \times(-\pi, \pi)\right) \mid u(R, \cdot)=0, u(\cdot,+\pi)=u(\cdot,-\pi)\right\},
$$

the truncation radius $R_{\mathrm{TR}} \in(0, R)$ enables to avoid a singular integrand (in $r$ ), and $\Theta_{m}$ and $\Theta_{d}$ are given by (5). In practice this formulation also suffers from a poor resolution of the critical interval as it prevents from choosing $R_{\mathrm{TR}} \ll R$, which is necessary to capture the asymptotic behavior at $r \rightarrow 0$. This issue is fixed by the second and preferred alternative that consists in using the so-called Euler coordinates $(z, \theta)$ with $z=\ln r$ [17], for which (39) writes: find $(u, \kappa) \in H_{e}^{1} \times \mathbb{C}$ such that for all $v \in H_{e}^{1}$,

$$
a_{S_{m}}^{(z, \theta)}(u, v)=-\kappa a_{S_{d}}^{(z, \theta)}(u, v), \text { where } a_{X}^{(z, \theta)}(u, v):=\int_{X}\left[\partial_{z} u \partial_{\theta} u\right] \cdot\left[\partial_{z} v \partial_{\theta} v\right] \mathrm{d} z \mathrm{~d} \theta
$$

and

$$
S:=\left(\ln R_{\mathrm{TR}}, \ln R\right) \times(-\pi, \pi), S_{m}:=\left(\ln R_{\mathrm{TR}}, \ln R\right) \times \Theta_{m}, S_{d}:=S \backslash \overline{S_{m}}, H_{e}^{1}:=\left\{\begin{array}{l|l}
u \in H^{1}(S) & \begin{array}{l}
u(\ln R, \cdot)=0 \\
u(\cdot,+\pi)=u(\cdot,-\pi)
\end{array}
\end{array}\right\} .
$$

Since $I_{c}$ is associated with functions that behave as $z \mapsto e^{i \eta_{c} z}$ towards $z=-\infty, \eta_{c}$ can be interpreted as a wavenumber. This observation leads to the following two remarks:

- The smaller the truncation radius $R_{\mathrm{TR}}$, the smaller the minimum well-resolved $\eta$. In practice, if a well-refined mesh gives a poor resolution of $I_{c}$ near the endpoints $\kappa_{\phi}^{ \pm 1}$, then $R_{\mathrm{TR}}$ should be reduced (this follows from the fact that $\eta_{c} \rightarrow 0$ when $\left.\kappa \rightarrow \kappa_{\phi}^{ \pm 1}\right)$.

- The smaller the mesh element size, the larger the maximum well-resolved $\eta_{c}$. In practice, the mesh is chosen coarse enough to avoid an overly dense cluster of eigenvalues at $\kappa=-1$. Indeed, a uniform mesh refinement leads to a non-uniform refinement of $I_{c}$, clustered at $\kappa=-1$ (since $\left|\eta_{c}\right| \rightarrow \infty$ when $\kappa \rightarrow-1$ ).

The center and right plots of Figure 15 illustrate the mesh and the resolution gain that can be achieved compared to the Cartesian mesh. Figure 16 plots an eigenfunction associated with the discretized critical interval, which highlights one additional advantage of discretizing in Euler coordinates: it will make it easier to identify eigenvalues associated with the critical interval.

\section{Corner with complex scaling}

Let us now consider the corner with a complex-scaling region of radius $R_{\alpha}$ depicted in Figure $14 \mathrm{~b}$. Following Section 3.1, the PEP $\alpha$ is naturally formulated in polar coordinates: find $(u, \kappa) \in H_{p}^{1} \times \mathbb{C}$ such that for all $v \in H_{p}^{1}$,

$$
\begin{aligned}
\int_{\Theta_{m}}\left[\int_{R_{\alpha}}^{R} \nabla u \cdot \nabla v r \mathrm{~d} r\right. & \left.+\alpha \int_{R_{\mathrm{TR}}}^{R_{\alpha}} \partial_{r} u \partial_{r} v r \mathrm{~d} r+\frac{1}{\alpha} \int_{R_{\mathrm{TR}}}^{R_{\alpha}} \partial_{\theta} u \partial_{\theta} v \frac{\mathrm{d} r}{r}\right] \mathrm{d} \theta= \\
& -\kappa \int_{\Theta_{d}}\left[\int_{R_{\alpha}}^{R} \nabla u \cdot \nabla v r \mathrm{~d} r+\alpha \int_{R_{\mathrm{TR}}}^{R_{\alpha}} \partial_{r} u \partial_{r} v r \mathrm{~d} r+\frac{1}{\alpha} \int_{R_{\mathrm{TR}}}^{R_{\alpha}} \partial_{\theta} u \partial_{\theta} v \frac{\mathrm{d} r}{r}\right] \mathrm{d} \theta,
\end{aligned}
$$




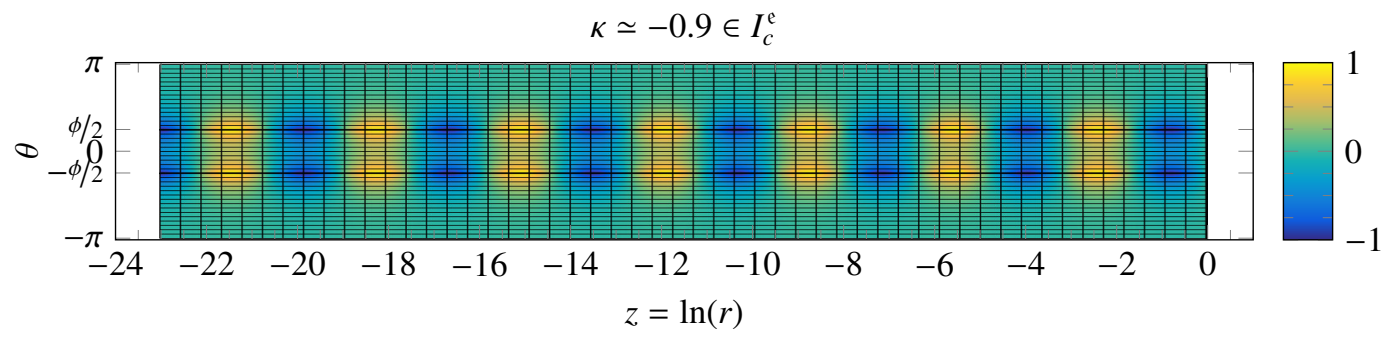

Fig. 16: Eigenfunction $\mathfrak{R}(u) /\|u\|_{\infty}$ computed using (40) for the corner geometry depicted in Figure $14 \mathrm{~b}$ with $\phi=\pi / 2, R=1, R_{\mathrm{TR}}=10^{-10}$ $\left(\ln R_{\mathrm{TR}} \simeq-23.02\right)$, and $N_{h}=8424$.

which writes in Euler coordinates: find $(u, \kappa) \in H_{e}^{1} \times \mathbb{C}$ such that for all $v \in H_{e}^{1}$,

$$
a_{S_{m} \backslash S_{\alpha}}^{(z, \theta)}(u, v)+\alpha a_{S_{m} \cap S_{\alpha}}^{(z)}(u, v)+\frac{1}{\alpha} a_{S_{m} \cap S_{\alpha}}^{(\theta)}(u, v)=-\kappa\left[a_{S_{d} \backslash S_{\alpha}}^{(z, \theta)}(u, v)+\alpha a_{S_{d} \cap S_{\alpha}}^{(z)}(u, v)+\frac{1}{\alpha} a_{S_{d} \cap S_{\alpha}}^{(\theta)}(u, v)\right],
$$

where $S_{\alpha}:=\left(\ln R_{\mathrm{TR}}, \ln R_{\alpha}\right) \times(-\pi, \pi)$ and

$$
a_{X}^{(z)}(u, v):=\int_{X} \partial_{z} u \partial_{z} v \mathrm{~d} z \mathrm{~d} \theta, a_{X}^{(\theta)}(u, v):=\int_{X} \partial_{\theta} u \partial_{\theta} v \mathrm{~d} z \mathrm{~d} \theta
$$

Discretizing as in Section 4.1 yields the $N_{h}$-dimensional linear eigenproblem

$$
\left[A_{S_{m} \backslash S_{\alpha}}^{(z, \theta)}+\alpha A_{S_{m} \cap S_{\alpha}}^{(z)}+\frac{1}{\alpha} A_{S_{m} \cap S_{\alpha}}^{(\theta)}\right] U=-\kappa\left[A_{S_{d} \backslash S_{\alpha}}^{(z, \theta)}+\alpha A_{S_{d} \cap S_{\alpha}}^{(z)}+\frac{1}{\alpha} A_{S_{d} \cap S_{\alpha}}^{(\theta)}\right] U
$$

where all the involved matrices are real, symmetric, and positive (but not definite): when $\alpha \notin \mathbb{R}$ the problem is thus complex-symmetric but not Hermitian.

Figure 17 plots the spectra computed for $\phi=0.9 \pi$ and two scaling parameters. It shows that a satisfactory resolution of the critical curves (31) can be achieved. The corresponding eigenfunctions behave as $e^{i \frac{\eta}{\alpha} z}$ for some $\eta / \alpha \in \mathbb{R}$, so they are similar to the one shown in Figure 16. The next and last section relies on this formulation to compute $\mathrm{CP}$ resonances.
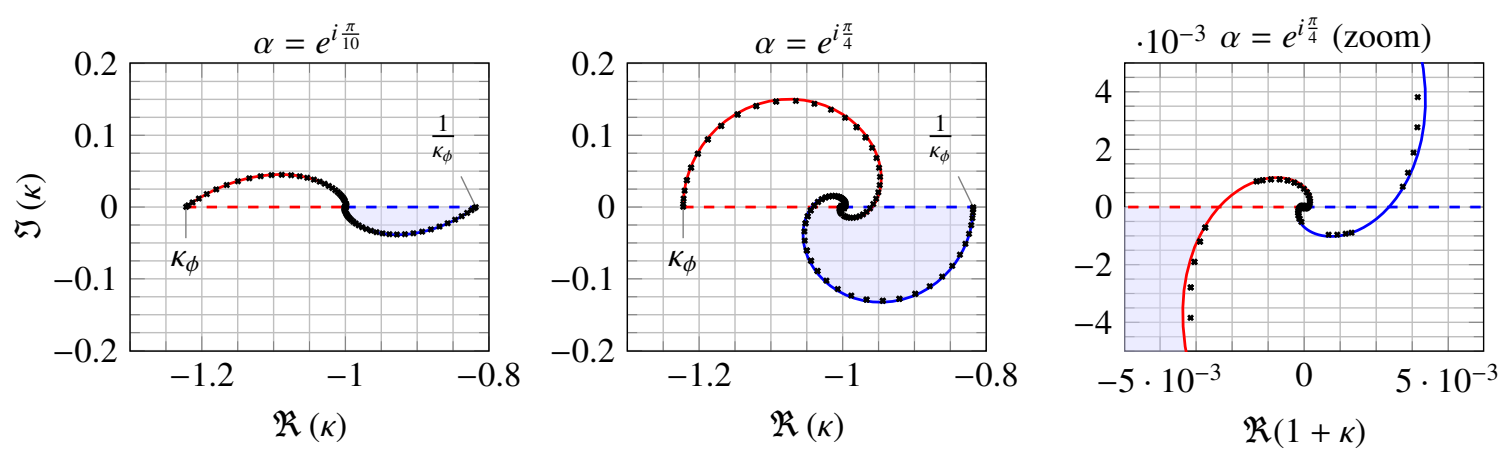

Fig. 17: Comparison between the critical curves (31) and the spectra computed using (42) for the corner geometry depicted in Figure 14b with $\phi=0.9 \pi, R=1, R_{\mathrm{TR}}=10^{-20}$, and $N_{h}=9888$. (一): even critical curve $I_{c}^{\mathrm{e}, \alpha},\left(\right.$ (一): $I_{c}^{\mathrm{v}, \alpha}$. (Left) $\alpha=e^{i \frac{\pi}{10}}$. (Center, Right) $\alpha=e^{i \frac{\pi}{4}}$. 

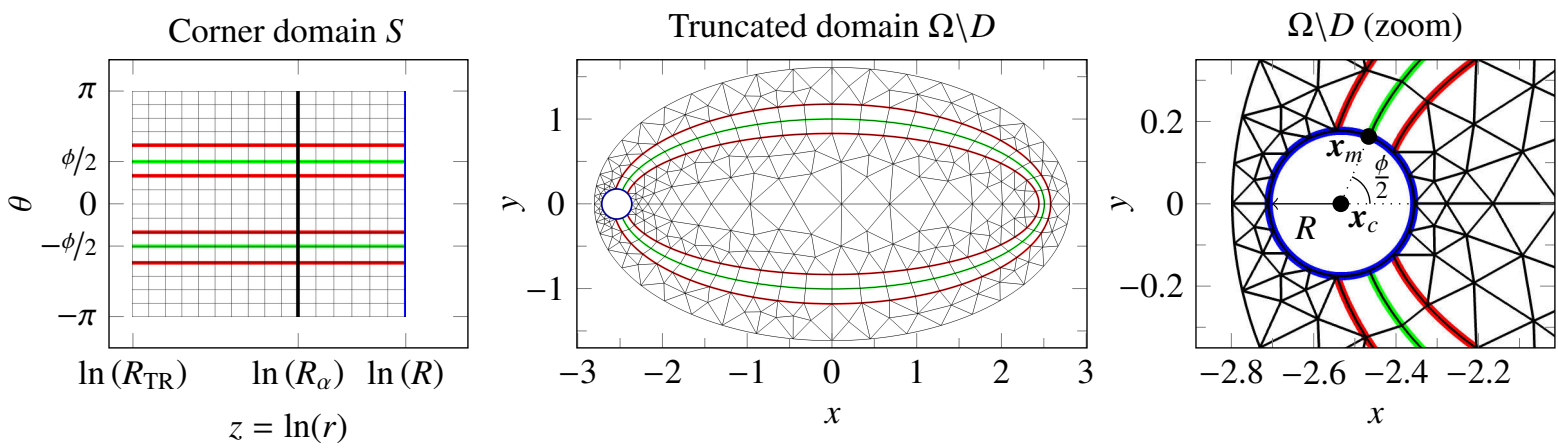

Fig. 18: Mesh topology for a particle $\Omega_{m}$ whose piecewise-smooth boundary $\partial \Omega_{m}$ (green) is an ellipse perturbed by a corner of angle $\phi \in(0, \pi)$. (Left) Corner domain $S$, structured mesh. (Center, Right) Domain $\Omega$ truncated around $\boldsymbol{x}_{c}$, mesh with structured layer defined by (38).

\section{Complex plasmonic resonances of elliptical particles perturbed by a corner}

This section gathers numerical results that illustrate the existence of CP resonances for elliptical particles perturbed by a corner. Section 5.1 describes the particle and its discretization, which mixes Cartesian and Euler coordinates. Section 5.2 discusses the numerical results and shows the agreement with the mechanism described in $[35, \S 5.2]$.

\subsection{Corner perturbation and weak formulation}

We consider an open set $\Omega_{m}$ whose piecewise-smooth boundary is an ellipse of semi-axes $\left(a_{m}, b_{m}\right)$ perturbed by a corner of angle $\phi \in(0, \pi)$ that is symmetric with respect to the major axis, see Figure 1a. The boundary $\partial \Omega_{m}$ is uniquely defined by $\left(a_{m}, b_{m}, \phi\right)$ since we deduce the remaining geometrical parameters, namely $\boldsymbol{x}_{c}:=\left(x_{c}, 0\right)$ and the top junction point $\boldsymbol{x}_{m}:=\left(x_{m}, y_{m}\right)$, by imposing a $C^{1}$ junction (see the right graph of Figure 18):

$$
\left(\frac{x_{m}}{a_{m}}\right)^{2}+\left(\frac{y_{m}}{b_{m}}\right)^{2}=1, x_{m}=-a_{m} \frac{\tan \left(\frac{\phi}{2}\right)}{\sqrt{\tan \left(\frac{\phi}{2}\right)^{2}+\left(\frac{b_{m}}{a_{m}}\right)^{2}}}, x_{c}=x_{m}-\frac{y_{m}}{\tan \left(\frac{\phi}{2}\right)} .
$$

The smaller $\pi-\phi$, the smaller the perturbation radius $R:=\left|\boldsymbol{x}_{c}-\boldsymbol{x}_{m}\right|$. The sets $\Omega$ and $\Omega_{d}$ are defined as in (35). Following the results of Section 4 , we formulate the discrete problem on the truncated set $\Omega \backslash D_{\mathrm{TR}}$ where $D_{\mathrm{TR}}:=$ $\left\{\left|\boldsymbol{x}-\boldsymbol{x}_{c}\right|<R_{\mathrm{TR}}\right\}$ with $R_{\mathrm{TR}} \in(0, R]$. The mesh is split into two parts, see Figure 18: the subset $D \backslash D_{\mathrm{TR}}$ is meshed in Euler coordinates while the subset $\Omega \backslash D$ is meshed in Cartesian coordinates with a structured layer at the sign-changing interface $\partial \Omega_{m}$ defined using (38).

The weak formulation is: find $\left(\left(u_{\alpha}, \breve{u}_{\alpha}\right), \kappa\right) \in H_{c, e}^{1} \times \mathbb{C}$ such that for all $(v, \breve{v}) \in H_{c, e}^{1}$,

$$
\begin{aligned}
a_{\Omega_{m} \backslash D}^{(x, y)}\left(u_{\alpha}, v\right)+ & a_{S_{m} \backslash S_{\alpha}}^{(z, \theta)}\left(\breve{u}_{\alpha}, \breve{v}\right)+\alpha a_{S_{m} \cap S_{\alpha}}^{(z)}\left(\breve{u}_{\alpha}, \breve{v}\right)+\frac{1}{\alpha} a_{S_{m} \cap S_{\alpha}}^{(\theta)}\left(\breve{u}_{\alpha}, \breve{v}\right)= \\
& -\kappa\left[a_{\Omega_{d} \backslash D}^{(x, y)}\left(u_{\alpha}, v\right)+a_{S_{d} \backslash S_{\alpha}}^{(z, \theta)}\left(\breve{u}_{\alpha}, \breve{v}\right)+\alpha a_{S_{d} \cap S_{\alpha}}^{(z)}\left(\breve{u}_{\alpha}, \breve{v}\right)+\frac{1}{\alpha} a_{S_{d} \cap S_{\alpha}}^{(\theta)}\left(\breve{u}_{\alpha}, \breve{v}\right)\right],
\end{aligned}
$$

with

$$
H_{c, e}^{1}:=\left\{\begin{array}{l|l}
(u, \breve{u}) \in H^{1}(\Omega \backslash \bar{D}) \times H^{1}(S) & \begin{array}{l}
u_{\mid \partial \Omega}=0, \breve{u}(z,+\pi)=\breve{u}(z,-\pi) \quad\left(z \in\left(\ln R_{\mathrm{TR}}, \ln R\right)\right) \\
u\left(x_{c}+R \cos \theta, R \sin \theta\right)=\breve{u}(\ln R, \theta) \quad(\theta \in(-\pi, \pi])
\end{array}
\end{array}\right\} .
$$

This formulation enforces a Neumann boundary condition at the truncation $z=\ln R_{\mathrm{TR}}$. As in Section 4, we employ a mix of $\mathbb{P}_{2}$ and $\mathbb{Q}_{2}$ isoparametric Lagrange elements to obtain, after eliminating the DoF on $\partial \Omega$, a $N_{h}$-dimensional linear generalized eigenvalue problem.

Remark 26 (On the choice of a bounded $\Omega_{d}$ ). The definitions and results of Sections 2 and 3 carry over to the case where $\Omega_{d}$ is bounded, since they rely on a local analysis in a neighborhood of the corner. Using a Dirichlet boundary condition on $\partial \Omega$ will enable us to keep the discussion focused on the corner discretization; using instead a Dirichletto-Neumann operator would add a $\kappa$-independent term to (43), which if discretized adequately would not change the conclusions (the formula for $\kappa_{n}^{\mathrm{e}(\mathfrak{D})}$ (D.1) still applies to the unbounded case). 


\subsection{Numerical results}

Throughout this section the parameters of the unperturbed ellipse are

$$
a_{m}=2.5, b_{m}=1, c:=\sqrt{a_{m}^{2}-b_{m}^{2}} .
$$

We will consider two geometrical cases (A and B) that differ by both the angle $\phi$ of the corner perturbation and the semi-axes $\left(a_{d}, b_{d}\right)$ of the outer ellipse $\partial \Omega$. Note that the semi-major axis $a_{d}$ limits the perturbation radius $R$ through the constraint $\left|x_{c}\right|+R<a_{d}$ (see right plot of Figure 18), so that for a given $a_{d}$ not all corner angles $\phi$ are achievable.

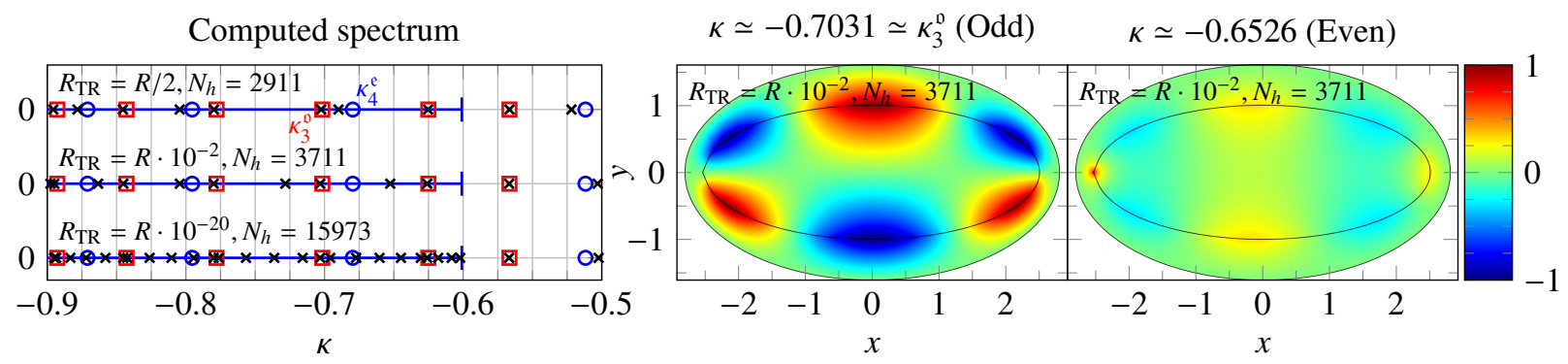

Fig. 19: PEP (43) for the case A parameters (44) and $\alpha=1$. (Left) $(\times)$ : computed, $(\bigcirc): \kappa_{n}^{\mathrm{e}},(\square): \kappa_{n}^{\mathrm{D}},(-)$ : $I_{c}^{\mathrm{e}}$. (Center, Right) Computed eigenfunctions $u /\|u\|_{\infty}$ associated with a contrast inside $I_{c}^{\mathrm{e}}$. The corner domain $S$ has been mapped to ease the visualization.

\section{Case A: computation of complex plasmonic resonances}

Key to the construction of CP resonances is the local symmetry at the corner, which here (by design) coincides with the symmetry with respect to the major axis. The purpose of this first numerical experiment is to highlight the different behaviors of even and odd eigenfunctions when the corner perturbation is applied. To this end, we choose the geometrical parameters as follows:

$$
\phi=0.75 \pi, a_{d}=\left|x_{c}\right|+1.5 R, b_{d}=\sqrt{a_{d}^{2}-c^{2}} .
$$

The corresponding outer ellipse $\partial \Omega$ satisfies $\mu_{d} \in\left(\mu_{m}, 2 \mu_{m}\right)$ so that all the exact unperturbed eigenvalues $\kappa_{n}^{\mathrm{e}}$ and $\kappa_{n}^{\mathfrak{D}}$ belong to $(-1,0]$, see (D.1). As a result, we will focus our interest in the region $\{\mathfrak{R}(\kappa)>-1\}$.

Let us first consider the PEP (43) without complex scaling, i.e. $\alpha=1$. If $R_{\mathrm{TR}}=R$, the particle $\Omega_{m}$ is an ellipse truncated by a disc of radius $R_{\mathrm{TR}}$ centered on $x_{c}$; if $R_{\mathrm{TR}}<R$, the particle is an ellipse perturbed by a truncated corner. In the limit $R_{\mathrm{TR}} \rightarrow 0$ the truncated corner tends to a corner. Figure 19 highlights the different behaviors of odd and even eigenfunctions as $R_{\mathrm{TR}}$ is reduced. The left graph shows the eigenvalues computed for various $R_{\mathrm{TR}}$. Overall, odd eigenvalues are significantly less perturbed than their even counterparts. As $R_{\mathrm{TR}}$ is reduced, even eigenvalues in $I_{c}^{\mathrm{e}}$ gets significantly perturbed, their density increasing so as to progressively form $I_{c}^{\mathrm{e}}$. By contrast, even eigenvalues outside $I_{c}$ remain isolated regardless of the value of $R_{\mathrm{TR}}$.

The center and right graphs of Figure 19 plot two eigenfunctions obtained for a relatively large truncation radius $R_{\mathrm{TR}}=10^{-2} \cdot R$, which ensures that the corresponding eigenvalues are clearly isolated in the computed spectrum. This illustrates that while odd eigenfunctions undergo very little perturbation when $R_{\mathrm{TR}}$ is reduced, even eigenfunctions concentrate at the corner in an increasingly singular fashion.

Intuitively, using a suitable $\alpha \neq 1$ will dissipate energy at the corner and tame the singularity. Figure 20 plots the spectra computed for various values of $\arg (\alpha)$. The radius $R_{\mathrm{TR}}=10^{-50}$ is chosen to ensure a well-resolved critical curve, but such a small value is not necessary to obtain $\mathrm{CP}$ resonances in this case. A first observation is that the eigenvalues $\kappa_{n}^{\mathfrak{D}}$ that belong to $I_{c}^{\mathrm{e}}$ have stayed real and have been perturbed into even-critical EP eigenvalues. This can be justified by noting that the corresponding eigenfunctions are odd and therefore their local expansions (28) cannot include the even black-hole wave $u_{\eta_{\mathrm{bh}}^{\mathrm{e}}}$. The bottom row of Figure 21 plots the complex-scaled eigenfunction $\breve{u}_{\alpha}$ associated with the contrast $\kappa \simeq \kappa_{3}^{\mathfrak{D}}$, which satisfies $\breve{u}_{\alpha}(z, \theta) \underset{z \rightarrow-\infty}{\rightarrow} 0$.

By contrast, the eigenvalues $\kappa_{n}^{\mathrm{e}}$ that belong to $I_{c}^{\mathrm{e}}$ have not stayed real and have been perturbed into even-critical CP resonances: four of them can be seen in Figure 20, which have arisen from $\kappa_{4}^{\mathrm{e}}, \kappa_{5}^{\mathrm{e}}, \kappa_{6}^{\mathrm{e}}$, and $\kappa_{7}^{\mathrm{e}}$. The local expansions 

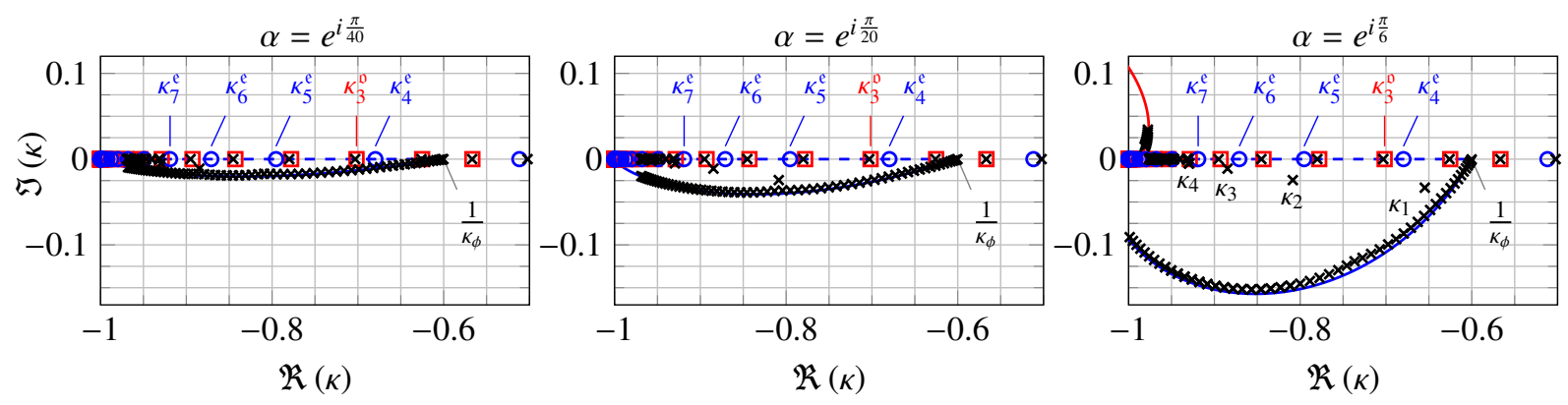

Fig. 20: PEP (43) for the case A parameters (44) and increasing values of $\arg (\alpha)$. $(\times)$ : computed $\left(N_{h}=26345, R_{\alpha}=R / 2, R_{\mathrm{TR}}=10^{-50} \cdot R\right)$, (○): unperturbed even eigenvalues $\kappa_{n}^{\mathrm{e}},(\square): \kappa_{n}^{\mathfrak{0}},(-)$ : even critical curve $I_{c}^{\mathrm{e}, \alpha},(-): I_{c}^{\mathrm{v}, \alpha}$.

(Right part of) Domain $S$

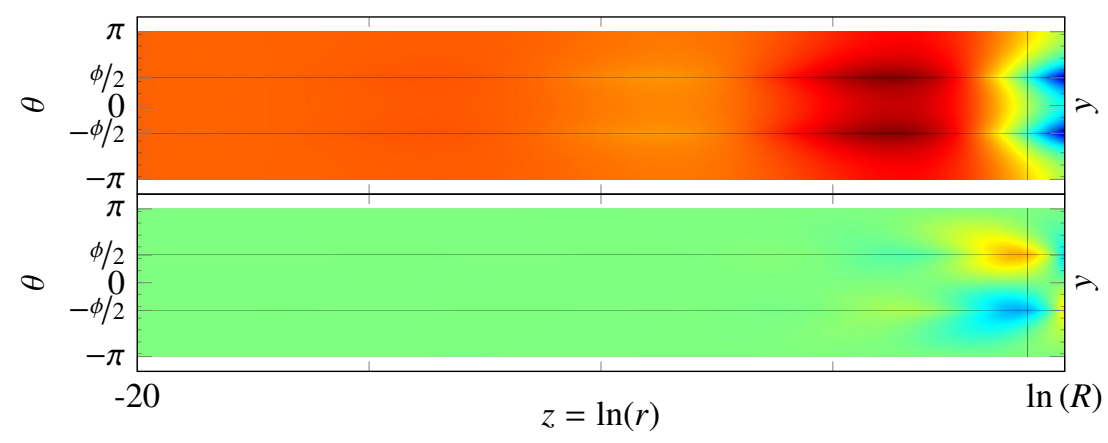

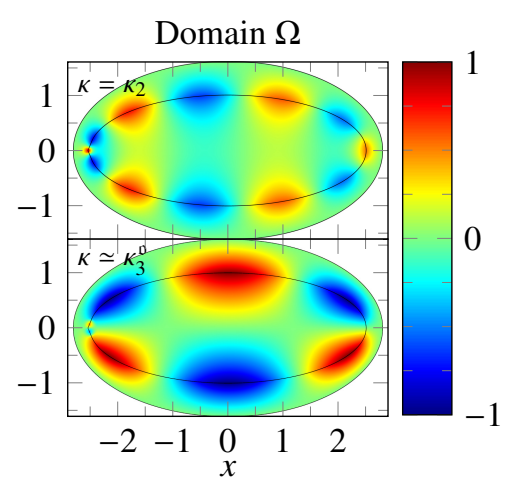

Fig. 21: Eigenfunctions $\mathfrak{R}\left(u_{\alpha}\right) /\left\|u_{\alpha}\right\|_{\infty}$ of PEP (43) associated with Figure 20 ( $\left.\alpha=e^{i \frac{\pi}{6}}\right)$. (Top row) $\kappa=\kappa_{2} \simeq 0.8086-0.02445 i$, even-critical CP resonance. (Bottom row) $\kappa \simeq 0.70313-8.0357 \cdot 10^{-8} i \simeq \kappa_{3}^{\mathfrak{D}}$, even-critical EP eigenvalue.

(28) of the associated CP resonance functions include a non-null contribution from the even black-hole wave, so they blow up like (29). The complex-scaled resonance functions behave as $\breve{u}_{\alpha}(z, \theta) \underset{z \rightarrow-\infty}{\rightarrow} c_{0}$ since the exponentially growing term is dissipated in the complex-scaling region $S_{\alpha}$; this is illustrated in the top row of Figure 21 for $\kappa=\kappa_{2}$. The behavior of $\mathrm{CP}$ resonance functions is as follows: the larger $|\mathfrak{J}(\kappa)|$, the larger $|\mathfrak{J}(\eta)|$, so the stronger the corner singularity; the smaller $|\kappa+1|$, the larger $|\mathfrak{R}(\eta)|$, so the stronger the oscillation as $r \rightarrow 0$.

The convergence of the computed CP resonances is plotted in the left graph of Figure 22, where the exact values are approximated as those obtained on a fine mesh and the $x$-axis is normalized with the value $N_{h}=26345$ used in Figures 20 and 21. The closer a CP resonance is to -1 , the larger its approximation error. The achieved asymptotic convergence rate can be estimated as $O\left(N_{h}^{-3 / 2}\right)$. The center and right plots of Figure 22 illustrate the sensitivity to $\arg (\alpha)$ for $N_{h}=26345$. These plots emphasize that each CP resonance can only be computed for $\arg (\alpha) \in\left(\theta_{\min }, \theta_{\max }\right)$, where $\theta_{\min }$ (resp. $\theta_{\max }$ ) is the value at which the CP resonance enters (resp. leaves) the uncovered region. For example, $\theta_{\min } \in(\pi / 10, \pi / 6)$ for $\kappa_{1}$ and $\theta_{\max } \in(\pi / 4, \pi / 3)$ for $\kappa_{2}$ and $\kappa_{3}$.

\section{Case B: dependence on perturbation radius}

The purpose of this second numerical experiment is to illustrate the path taken by $\mathrm{CP}$ resonances as the perturbation radius $R$ is varied (equivalently: as the corner angle $\phi$ is varied). Compared to case A we do not change $a_{m}$ and $b_{m}$ but we enlarge $\partial \Omega$ so that it can contain the largest corner perturbation considered. Specifically, the chosen geometrical parameters are

$$
\frac{\phi}{\pi} \in[0.63,0.86], a_{d}=\left[\left|x_{c}\right|+1.5 R\right]_{\mid \phi=0.63 \pi} \simeq 3.0409, b_{d}=\sqrt{a_{d}^{2}-c^{2}} .
$$

Since $a_{d}$ is larger than in case A, the exact unperturbed eigenvalues $\kappa_{n}^{\mathrm{e}(\mathfrak{o})}$ are different; in particular, the unperturbed eigenvalues $\kappa_{n}^{\mathfrak{0}}$ are closer to -1 . However, we are still in a case where all $\kappa_{n}^{\mathrm{e}}$ and $\kappa_{n}^{\mathrm{o}}$ belong to $(-1,0]$. 

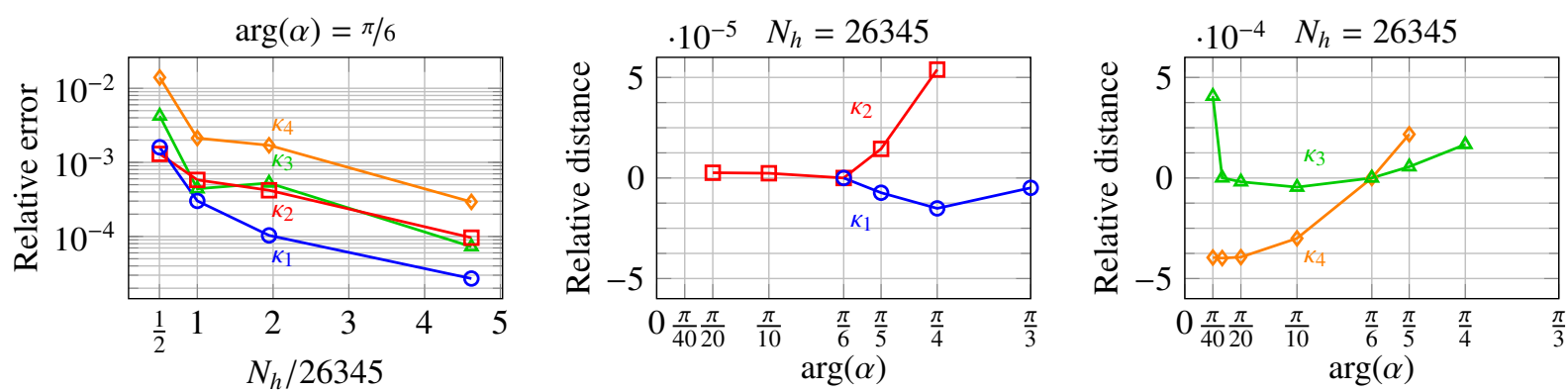

Fig. 22: CP resonances highlighted in Figure 20. (Left) Relative error $\mid \mathfrak{J}\left(\kappa_{n}-\kappa_{n}\right.$,fine $)|/| \mathfrak{J}\left(\kappa_{n}\right.$,fine $) \mid$ where $\kappa_{n}$,fine is the value of $\kappa_{n}$ computed with $N_{h}=3,976,233 . N_{h}$ is varied by changing the mesh in both $S$ and $\Omega \backslash D$, in order to control the maximum element growth rate. (Center and Right) Relative distance $\mid \mathfrak{I}\left(\kappa_{n}-\kappa_{n}\right.$,ref $)|/| \mathfrak{I}\left(\kappa_{n, \text { ref }}\right) \mid$ where $\kappa_{n}$,ref is the value of $\kappa_{n}$ at $\alpha=e^{i \frac{\pi}{6}}$.
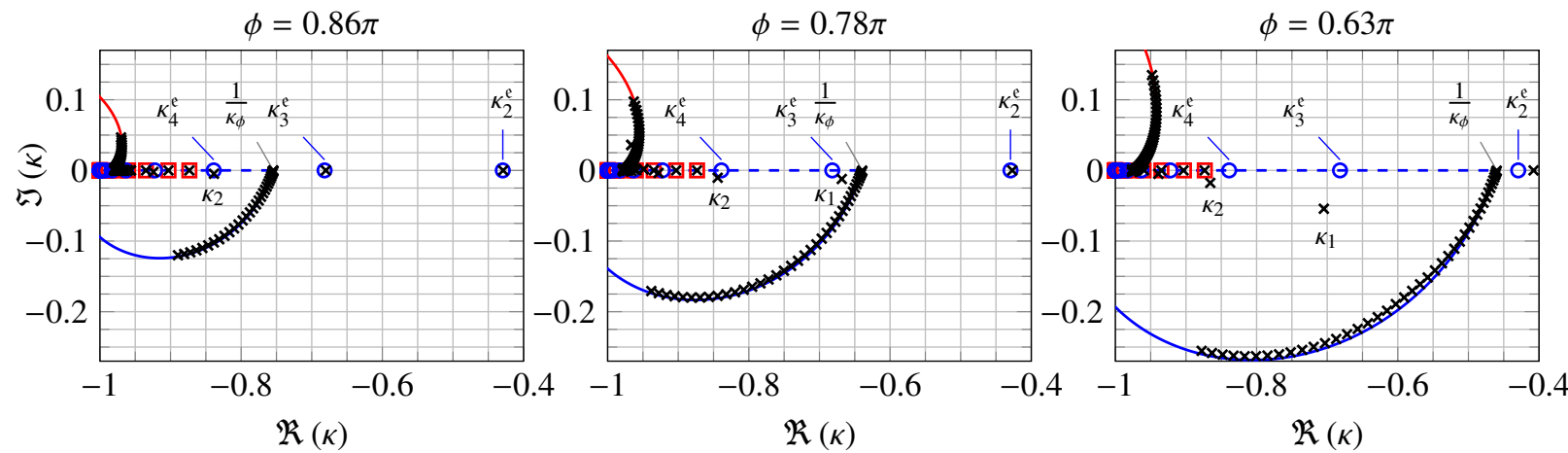

Fig. 23: PEP (43) for the case B parameters (45) and increasing perturbation radius $R$ (i.e. decreasing corner angle $\phi)$. $(\mathbf{x})$ : computed $\left(N_{h}=35695\right.$, $\left.R_{\alpha}=R / 2, R_{\mathrm{TR}}=10^{-50} \cdot R\right),(\bigcirc)$ : unperturbed even eigenvalues $\kappa_{n}^{\mathrm{e}},(\square): \kappa_{n}^{\mathrm{v}},(-)$ : even critical curve $I_{c}^{\mathrm{e}, \alpha},(-): I_{c}^{\mathrm{v}, \alpha}$.

The results are shown in Figure 23, where $\alpha=e^{i \pi / 5}$ has been used. As the perturbation radius is increased, the even critical interval $I_{c}^{\mathrm{e}}$ also increases. Once the eigenvalue $\kappa_{3}^{\mathrm{e}}$ is reached by $I_{c}^{\mathrm{e}}$, it gets perturbed into the even-critical CP resonance $\kappa_{1}$, which strays away from the real axis as $R$ is further increased. This behavior differs from that of the eigenvalue $\kappa_{2}^{\mathrm{e}}$, which is not reached by $I_{c}^{\mathrm{e}}$ and is thus always perturbed into a real eigenvalue.

The observations made in this section, namely that odd eigenvalues $\kappa_{n}^{\mathrm{D}}$ in $I_{c}^{\mathrm{e}}$ are perturbed into even-critical EP eigenvalues while even eigenvalues $\kappa_{n}^{\mathrm{e}}$ in $I_{c}^{\mathrm{e}}$ are perturbed into even-critical CP resonances, are in agreement with the mechanism described in $[35, \S 5.2]$.

\section{Conclusion and outlook}

This paper has investigated the existence of complex plasmonic resonances for a 2D subwavelength particle whose boundary is smooth except for one straight corner.

Section 2 has proposed a definition of complex plasmonic resonances (Definition 17) analogous to that of complex scattering resonances, with the local behavior at the corner playing the role of the behavior at infinity; in particular, complex scattering resonance functions blow up at infinity while complex plasmonic resonance functions blow up at the corner. The construction of the definition has crucially relied on the multivalued nature of the set of stable exponents (Proposition 12).

Section 3 has shown that isolated eigenvalues of the complex-scaled PEP (Definition 21) that lie inside the socalled uncovered region are embedded plasmonic eigenvalues or complex plasmonic resonances (Proposition 22). The employed complex scaling has been introduced in [17] and can be described as applying a radial scaling in a neighborhood of the corner, see (30). Figure 17 has illustrated how this complex scaling deforms the critical interval, unveiling part of another Riemann sheet where complex plasmonic resonances may be computed.

A standard FE discretization of the complex-scaled PEP has led us to a (complex-symmetric) linear generalized eigenvalue problem (43), which is the key computational feature of this technique. However, as has been emphasized 
in Section 4, the main drawback is the stringent constraint on mesh symmetry at the sign-changing interface.

The numerical results of Section 5 have illustrated the existence of both embedded plasmonic eigenvalues and complex plasmonic resonances for elliptical particles perturbed by a corner. Surface plasmons associated with embedded plasmonic eigenvalues are as regular as surface plasmons associated with isolated plasmonic eigenvalues, while surface plasmons associated with complex plasmonic resonances blow up at the corner like (29). The computed spectra are in agreement with the results presented in [34, Fig. 6], obtained with a rate-of-resonance criterion applied in conjunction with a BE method, which highlighted the presence of embedded plasmonic eigenvalues. In addition, the results also corroborate the mechanism proposed by [35, § 5.2]: even (odd) eigenvalues of the unperturbed ellipse become complex plasmonic resonances if covered by the even (odd) critical interval, while they become embedded plasmonic eigenvalues if covered by the odd (even) critical interval.

Let us mention ideas for future work. Firstly, it would be interesting to drop the quasi-static approximation and compute complex plasmonic resonances using the Helmholtz equation with Drude's model, which yields a nonlinear PEP. Secondly, the computational interest of using a $\kappa$-dependent scaling parameter in (43) could be studied, similarly to what has been done in [60] for scattering problems. Thirdly, the 3D extension of the employed complex scaling could be considered: a meaningful starting point would be the rotationally symmetric domains with conical point considered in [61, 62]. Lastly, the design of an alternative method to compute complex plasmonic resonances would enable an interesting comparison with the FE results presented here. A BE method might be a natural candidate, due to the widespread use of such methods to discretize the PEP [24, 25]; however this is an open problem.

\section{Acknowledgment}

This research has been partly financially supported by the Saclay regional center of INRIA.

\section{A. Corner dispersion relations}

This section recalls the derivation of the two corner dispersion relations (7), see also $[13,17]$. For any $\eta \in \mathbb{C}$, we define the orthoradial functions

$$
\begin{aligned}
\Phi_{\eta}^{\mathrm{e}}(\theta) & :=+\frac{\cosh [\eta \theta]}{\cosh \left[\eta \frac{\phi}{2}\right]} \mathbb{1}_{\Theta_{m}}(\theta)+\frac{\cosh [\eta(\pi-|\theta|)]}{\cosh \left[\eta\left(\pi-\frac{\phi}{2}\right)\right]} \mathbb{1}_{\Theta_{d}}(\theta), \\
\Phi_{\eta}^{\mathrm{v}}(\theta) & :=+\frac{\sinh [\eta \theta]}{\sinh \left[\eta \frac{\phi}{2}\right]} \mathbb{1}_{\Theta_{m}}(\theta)+\operatorname{sign}(\theta) \frac{\sinh [\eta(\pi-|\theta|)]}{\sinh \left[\eta\left(\pi-\frac{\phi}{2}\right)\right]} \mathbb{1}_{\Theta_{d}}(\theta),
\end{aligned}
$$

where $\Theta_{m}$ and $\Theta_{d}$ are given by (5), and $\mathbb{1}_{X}$ is the characteristic function of $X$. Note that $\Phi_{0}^{\mathfrak{e}}(\theta)=1$, while $\Phi_{0}^{\mathfrak{p}}$ is an odd piecewise-linear function.

Proposition 27 (Periodic transmission problem). Let $\phi \in(0,2 \pi) \backslash\{\pi\}, \kappa \in \mathbb{C}$, and $\sigma(\theta):=1 / \kappa \mathbb{1}_{\Theta_{m}}(\theta)+\mathbb{1}_{\Theta_{d}}(\theta)$, where $\Theta_{m}$ and $\Theta_{d}$ are given by (5). The $2 \pi$-periodic transmission problem

$$
\partial_{\theta}\left[\sigma(\theta) \partial_{\theta} u(\theta)\right]=\eta^{2} \sigma(\theta) u(\theta) \quad(\theta \in(-\pi, \pi))
$$

admits a solution if and only if $f_{\phi}^{\mathfrak{e}}(\eta, \kappa) \times f_{\phi}^{\mathfrak{0}}(\eta, \kappa)=0$.

- If $\eta \neq 0$ and $f_{\phi}^{\mathrm{e}(\mathfrak{p})}(\eta, \kappa)=0$, then $u(\theta)=C \Phi_{\eta}^{\mathrm{e}(\mathfrak{p})}(\theta)$.

- If $\eta=0$ then $f_{\phi}^{\mathrm{e}}(\eta, \kappa)=f_{\phi}^{\mathfrak{o}}(\eta, \kappa)=0$ and we have

$$
u(\theta)=C \Phi_{0}^{\mathrm{e}}(\theta) \quad \text { if } \kappa \neq \kappa_{\phi}, u(\theta)=C_{1} \Phi_{0}^{\mathrm{e}}(\theta)+C_{2} \Phi_{0}^{\mathfrak{p}}(\theta) \quad \text { if } \kappa=\kappa_{\phi} .
$$

Proof. The strong form of the transmission problem is

$$
u^{\prime \prime}(\theta)=\eta^{2} u(\theta) \quad(\theta \in(-\pi,-\phi / 2) \cup(-\phi / 2, \phi / 2) \cup(\phi / 2, \pi)),
$$

with boundary conditions

$$
u(\pi)=u(-\pi) \quad \text { (A) } \quad u^{\prime}(\pi)=u^{\prime}(-\pi)
$$




$$
\begin{array}{lll}
u_{\mid \Theta_{d}}(\phi / 2)=u_{\mid \Theta_{m}}(\phi / 2) & \text { (C) } \quad u_{\mid \Theta_{d}}(-\phi / 2)=u_{\mid \Theta_{m}}(-\phi / 2) \\
\kappa u_{\mid \Theta_{d}}^{\prime}(\phi / 2)=u_{\Theta_{m}}^{\prime}(\phi / 2) & \text { (E) } \quad \kappa u_{\mid \Theta_{d}}^{\prime}(-\phi / 2)=u_{\Theta_{m}}^{\prime}(-\phi / 2) .
\end{array}
$$

(Case $\eta=0$ ) Since a fundamental system of solutions is $\theta \mapsto(1, \theta)$, we seek $u$ as a piecewise-linear function

$$
u(\theta)= \begin{cases}A_{1}+B_{1} \theta & (\theta \in(-\phi / 2, \phi / 2)) \\ A_{2}+B_{2}(\theta-\pi) & (\theta \in(\phi / 2, \pi)) \\ A_{3}+B_{3}(\theta+\pi) & (\theta \in(-\pi,-\phi / 2))\end{cases}
$$

The periodicity conditions (A,B) give $A_{2}=A_{3}$ and $B_{2}=B_{3}$. The transmission conditions (E,F) give only one additional constraint $B_{1}=\kappa B_{2}$, so that there remain three DoF $A_{2}, B_{2}$, and $A_{1}$. The continuity conditions (C,D) give $A_{1}=A_{2}$ and $\left[\phi / 2-\pi-\kappa^{\phi / 2}\right] B_{2}=0$.

(Case $\eta \neq 0$ ) A fundamental system of solution is $\theta \mapsto(\cosh (\eta \theta)$, $\sinh (\eta \theta))$, so we seek $u$ as

$$
u(\theta)=u^{\mathrm{e}}(\theta)+u^{\mathfrak{D}}(\theta)= \begin{cases}A_{1} \cosh (\eta \theta)+B_{1} \sinh (\eta \theta) & (\theta \in(-\phi / 2, \phi / 2)) \\ A_{2} \cosh (\eta(\theta-\pi))+B_{2} \sinh (\eta(\theta-\pi)) & (\theta \in(\phi / 2, \pi)) \\ A_{3} \cosh (\eta(\theta+\pi))+B_{3} \sinh (\eta(\theta+\pi)) & (\theta \in(-\pi,-\phi / 2)) .\end{cases}
$$

The boundary conditions yield $A_{2}=A_{3}, B_{2}=B_{3}$, and the linear system

$$
\left[\begin{array}{cccc}
\cosh \left(\eta \frac{\phi}{2}\right) & -\cosh \left[\eta\left(\pi-\frac{\phi}{2}\right)\right] & 0 & 0 \\
\sinh \left(\eta \frac{\phi}{2}\right) & \kappa \sinh \left[\eta\left(\pi-\frac{\phi}{2}\right)\right] & 0 & 0 \\
0 & 0 & \sinh \left(\eta \frac{\phi}{2}\right) & \sinh \left[\eta\left(\pi-\frac{\phi}{2}\right)\right] \\
0 & 0 & \cosh \left(\eta \frac{\phi}{2}\right) & -\kappa \cosh \left[\eta\left(\pi-\frac{\phi}{2}\right)\right]
\end{array}\right]\left[\begin{array}{c}
A_{1} \\
A_{2} \\
B_{1} \\
B_{2}
\end{array}\right]=0
$$

This gives the even

$$
\kappa \cosh \left(\eta \frac{\phi}{2}\right) \sinh \left[\eta\left(\pi-\frac{\phi}{2}\right)\right]+\sinh \left(\eta \frac{\phi}{2}\right) \cosh \left[\eta\left(\pi-\frac{\phi}{2}\right)\right]=0
$$

and odd

$$
\kappa \sinh \left(\eta \frac{\phi}{2}\right) \cosh \left[\eta\left(\pi-\frac{\phi}{2}\right)\right]+\cosh \left(\eta \frac{\phi}{2}\right) \sinh \left[\eta\left(\pi-\frac{\phi}{2}\right)\right]=0
$$

dispersion relations. To obtain (7) we use the identity

$$
2\left(a_{1} b_{1}+a_{2} b_{2}\right)=\left(a_{1}+a_{2}\right)\left(b_{1}+b_{2}\right)+\left(a_{1}-a_{2}\right)\left(b_{1}-b_{2}\right)
$$

combined with the formula for $\sinh (a+b)[13, \S 4.1]$.

\section{B. Solutions of corner dispersion relations}

This section gathers the proofs for some of the results claimed in Sections 2.1 and 2.2.1.

\section{B.1. General properties}

Lemma 28 ( $\kappa$-invariant exponents). Let $\phi \neq \pi$. The set of even (odd) $\kappa$-invariant exponents satisfies

$$
\left\{\eta \in \mathbb{C} \mid \forall \kappa \in \mathbb{C}, \eta \in H_{\phi}^{\mathrm{e}(\mathfrak{o})}(\kappa)\right\}= \begin{cases}i q \mathbb{Z} & \text { when } \phi=\frac{p}{q} \pi \text { with } p \text { and } q \text { coprime integers } \\ \{0\} & \text { otherwise. }\end{cases}
$$

Proof. The set of $\eta$ satisfying both $\sinh [\eta \pi]=0$ and $\sinh [\eta(\pi-\phi)]=0$ is given by $i \times\left[\left(\frac{\pi}{\pi-\phi} \mathbb{Z}\right) \cap \mathbb{Z}\right]$.

Proof of Lemma 6. (a) For any $\kappa \in \mathbb{C}$, the map $\eta \mapsto f_{\phi}^{\mathrm{e}(\mathfrak{o})}(\eta, \kappa)$ is entire so its zeros are isolated and have no accumulation point in $\mathbb{C}$ [63, Thm. 10.18]. (b) Follows directly from the expression of $f_{\phi}^{\mathfrak{e}(\mathfrak{l})}$. (c) By definition of $f_{\phi}^{\mathfrak{e}(\mathfrak{)})}$, we have $0 \in H_{\phi}^{\mathrm{e}(\mathfrak{)})}(\kappa)$. If $\eta \in H_{\phi}^{\mathrm{e}}(\kappa) \cap H_{\phi}^{\mathfrak{o}}(\kappa)$, then $[1+\kappa] \sinh [\eta \pi]=0$ and $[1-\kappa] \sinh [\eta(\pi-\phi)]=0$. If $\kappa=1$, then $\eta \pi=i k \pi$ with $k \in \mathbb{Z}$. If $\kappa=-1$, then $\eta(\pi-\phi)=i k \pi$ with $k \in \mathbb{Z}$. If $\kappa \in \mathbb{C} \backslash\{-1,1\}$, then $\eta$ solves both $\sinh [\eta \pi]=0$ and $\sinh [\eta(\pi-\phi)]=0$, so that the intersection $H_{\phi}^{\mathrm{e}}(\kappa) \cap H_{\phi}^{\mathrm{o}}(\kappa)$ is exactly the set of $\kappa$-invariant exponents that is described in Lemma 28. 
Proof of Prop. 7 continued. It remains to prove that $\eta_{c}$, which is isolated from Lemma 6(a), crosses the real axis when $\kappa$ crosses $I_{c}^{\mathfrak{e}(\mathfrak{b})}$. Let $\left(\eta_{c}, \kappa_{c}\right) \in(0, \infty) \times I_{c}^{\mathrm{e}(\mathfrak{D})}$. Proposition 11 , which is a consequence of the implicit function theorem, gives the existence of a neighborhood $V$ of $\kappa_{c}$ in $\mathbb{C} \backslash\left\{\kappa_{\phi}, 1 / \kappa_{\phi}\right\}$ such that there is a unique analytic map $\eta: V \rightarrow \mathbb{C}$ that satisfies $\eta\left(\kappa_{c}\right)=\eta_{c}$ and $f(\eta(\kappa), \kappa)=0$ for all $\kappa \in V$. Since $\eta(\bar{\kappa})=\overline{\eta(\kappa)}, \eta(\kappa)$ must cross the real axis when $\kappa$ crosses the critical interval $I_{c}^{\mathrm{e}(\mathfrak{p})}$ in $V$.

Proof of Prop. 11. Let $\left(\eta_{0}, \kappa_{0}\right) \in \mathbb{C} \times U$ such that $f_{\phi}^{\mathrm{e}(\mathfrak{o})}\left(\eta_{0}, \kappa_{0}\right)=0$. The claim follows from the implicit function theorem [44, Prop. 2.14]: since $f_{\phi}^{\mathfrak{e}(\mathfrak{o})}$ is analytic on $\mathbb{C} \times U$, it is enough to show that $\partial_{\eta} f_{\phi}^{\mathfrak{e}(\mathfrak{o})}\left(\eta_{0}, \kappa_{0}\right) \neq 0$. We have

$$
\partial_{\eta} f_{\phi}^{\mathrm{e}(\mathfrak{o})}\left(\eta_{0}, \kappa_{0}\right)=\left[1+\kappa_{0}\right] \pi \cosh \left[\eta_{0} \pi\right]_{(+)}^{-}\left[1-\kappa_{0}\right](\pi-\phi) \cosh \left[\eta_{0}(\pi-\phi)\right] .
$$

Let us first cover the case $\kappa_{0} \in\{-1,1\}$. If $\kappa_{0}=-1$ (resp. $\left.\kappa_{0}=1\right)$ then $\sinh \left[\eta_{0}(\pi-\phi)\right]=0\left(\right.$ resp. $\left.\sinh \left[\eta_{0} \pi\right]=0\right)$ so that $\partial_{\eta} f_{\phi}^{\mathrm{e}(\mathfrak{)})}\left(\eta_{0}, \kappa_{0}\right) \neq 0$. Now assume that $\kappa_{0} \notin\{-1,1\}$; we show that $\partial_{\eta} f_{\phi}^{\mathrm{e}(\mathfrak{p})}\left(\eta_{0}, \kappa_{0}\right)=0 \Leftrightarrow \kappa_{0} \in\left\{\kappa_{\phi}, 1 / \kappa_{\phi}\right\}$. The system $f_{\phi}^{\mathfrak{e}(\mathfrak{l})}\left(\eta_{0}, \kappa_{0}\right)=0, \partial_{\eta} f_{\phi}^{\mathfrak{e}(\mathfrak{p})}\left(\eta_{0}, \kappa_{0}\right)=0$ reads

$$
\left\{\begin{array}{l}
\left.\left[1+\kappa_{0}\right] \sinh \left[\eta_{0} \pi\right]=\stackrel{+}{+}\right)_{+}\left[1-\kappa_{0}\right] \sinh \left[\eta_{0}(\pi-\phi)\right] \\
{\left[1+\kappa_{0}\right] \cosh \left[\eta_{0} \pi\right]={ }_{(-)}^{+}\left[1-\kappa_{0}\right](1-\phi / \pi) \cosh \left[\eta_{0}(\pi-\phi)\right],}
\end{array}\right.
$$

which is equivalent to

$$
\left[1+\kappa_{0}\right]=\stackrel{+}{(-)}\left[1-\kappa_{0}\right] \psi_{\phi}\left(\eta_{0}\right), \tanh \left[\eta_{0} \pi(1-\phi / \pi)\right]=(1-\phi / \pi) \tanh \left[\eta_{0} \pi\right] .
$$

Since the only solution of the rightmost equation is $\eta_{0}=0$, the first one is $\left[1+\kappa_{0}\right]={ }_{(-)}^{+}\left[1-\kappa_{0}\right] \frac{\pi-\phi}{\pi}$.

Proof of Prop. 12. The proof consists in showing that the set $H_{\phi}^{\mathrm{e}(\mathfrak{)})}(\kappa)$ contains a pair of multivalued elements, namely $\left(\eta_{c},-\eta_{c}\right)$ where $\eta_{c}$ is the critical exponent introduced in Proposition 7. Let us first consider the odd dispersion relation.

(1) Odd dispersion relation. Let $\kappa \in I_{c}^{\mathrm{p}}$. The (positive) critical exponent satisfies

$$
\frac{1}{\psi_{\phi}\left(\eta_{c}(\kappa)\right)}=\beta(\kappa)
$$

The critical exponent is a map $\eta_{c}: I_{c}^{\mathfrak{p}} \rightarrow(0, \infty)$ and Proposition 11 provides us with an analytic map $\tilde{\eta}_{c}: V \rightarrow \mathbb{C}$ such that $\tilde{\eta}_{c}(\kappa)=\eta_{c}$, where $V$ is a neighborhood of $\kappa$ in $\mathbb{C} \backslash\left\{\kappa_{\phi}, 1 / \kappa_{\phi}\right\}$. (a) The asymptotic expansion

$$
\frac{1}{\psi_{\phi}(\eta)} \underset{\eta \rightarrow 0}{=} \frac{\pi}{\pi-\phi}\left[1+\frac{1}{6} \eta^{2}\left(\pi^{2}-(\pi-\phi)^{2}\right)+O\left(|\eta|^{4}\right)\right]
$$

yields

$$
\left[\tilde{\eta}_{c}(\kappa)\right]^{2} \underset{\kappa \rightarrow \kappa_{\phi}}{=} \frac{6(1-\phi / \pi)}{\pi^{2}\left[1-(1-\phi / \pi)^{2}\right]}\left(\beta(\kappa)-\beta\left(\kappa_{\phi}\right)\right)+O\left(\left|\beta(\kappa)-\beta\left(\kappa_{\phi}\right)\right|^{2}\right) .
$$

By using the exact same reasoning as in Example 10, we deduce that $\kappa \mapsto \widehat{H}_{\phi}^{\mathrm{v}}(\kappa)$ has an algebraic branch point at $\kappa=\kappa_{\phi}$. (b) The asymptotic expansions

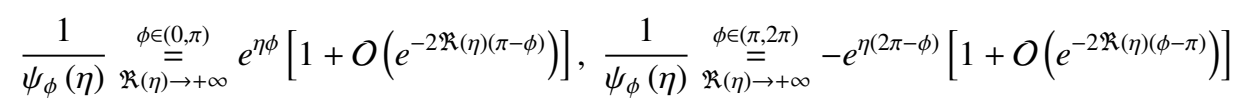

give

$$
\exp \left[\tilde{\eta}_{c}(\kappa) \phi\right] \underset{\kappa \rightarrow-1}{\stackrel{\phi \in(0, \pi)}{=}} \beta(\kappa)\left[1+O\left(\frac{1}{|\beta(\kappa)|^{2 \frac{\pi-\phi}{\phi}}}\right)\right], \exp \left[\tilde{\eta}_{c}(\kappa)(2 \pi-\phi)\right] \underset{\kappa \rightarrow-1}{\stackrel{\phi \in(\pi, 2 \pi)}{=}}-\beta(\kappa)\left[1+O\left(\frac{1}{|\beta(\kappa)|^{2 \frac{\phi-\pi}{2 \pi-\phi}}}\right)\right],
$$

from which we deduce that $\kappa \mapsto \widehat{H}_{\phi}^{\mathrm{v}}(\kappa)$ has a logarithmic branch point at $\kappa=-1$ (using again the same reasoning as in Example 10).

(2) Even dispersion relation. Identical to the odd case by replacing " $\beta$ " by " $-\beta$ ". The asymptotic expansions imply that $\kappa \mapsto \widehat{H}_{\phi}^{\mathrm{e}}(\kappa)$ has an algebraic branch point at $\kappa=1 / \kappa_{\phi}$ and a logarithmic branch point at $\kappa=-1$. 


\section{B.2. Closed form expression}

For any couple of integers $(p, q)$ such that $q \geq 2$ and $p / q \in(0,1) \cup(1,2)$, we define the following polynomial of degree $2(q-1)$ :

$$
P_{\beta}^{(p, q)}(x):=\sum_{k=0}^{q-1} x^{2 k}+\beta \operatorname{sign}(q-p) x^{q-|q-p|} \sum_{k=0}^{|q-p|-1} x^{2 k},
$$

whose roots are stable by inversion.

Proof of Prop. 9. Since $\kappa \neq-1$, the sets $H_{\phi}^{\mathrm{e}}(\kappa)$ and $H_{\phi}^{\mathrm{j}}(\kappa)$ are given by

$$
H_{\phi}^{\mathrm{e}(\mathfrak{o})}(\kappa)=\left\{\eta \in \mathbb{C} \mid \sinh (\eta \pi)={ }_{(+)}^{-} \beta(\kappa) \sinh [\eta(\pi-\phi)]\right\},
$$

where $\beta(\kappa)$ is given by (11). We carry out the proof only for $H_{\phi}^{\mathrm{e}}$; the proof for $H_{\phi}^{\mathrm{o}}$ is deduced with the substitution $\beta \rightarrow-\beta$. Let $x=\exp \left(\frac{\eta \pi}{q}\right)$. The statement $\eta \in H_{\phi}^{\mathrm{e}}(\kappa)$ is equivalent to the polynomial equation

$$
x^{2 q}-1=-\beta(\kappa)\left[x^{2 q-p}-x^{p}\right] .
$$

- If $\phi \in(0, \pi)$, then $0<p<q$ and we factor by $x^{p}$ to get $x^{2 q}-1=-\beta(\kappa) x^{p}\left[x^{2(q-p)}-1\right]$.

- If $\phi \in(\pi, 2 \pi)$, then $q<p<2 q$ and we factor by $x^{2 q-p}$ to get $x^{2 q}-1=+\beta(\kappa) x^{2 q-p}\left[x^{2(p-q)}-1\right]$.

In these two cases the claim is obtained using the identity $x^{2 n}-1=\left(x^{2}-1\right) \sum_{k=0}^{n-1} x^{2 k}$ on both sides and noting that $x^{2}=1$ has solutions $\eta \in i q \mathbb{Z}$. From Lemma 28 , the set $i q \mathbb{Z}$ is exactly the set of of $\kappa$-invariant exponents.

Example $29(\phi=\pi / 2) . P_{\beta}^{(1,2)}(x)=x^{2}+1+\beta x$ has roots given by $x_{\beta}^{ \pm}=\frac{-\beta \pm \sqrt{\beta^{2}-4}}{2}$, hence $\breve{H}_{\phi}^{\mathrm{e}}(\kappa)=\left\{\eta_{\beta(\kappa)}^{+}, \eta_{\beta(\kappa)}^{-}\right\}$and $\breve{H}_{\phi}^{\mathrm{v}}(\kappa)=\left\{\eta_{-\beta(\kappa)}^{+}, \eta_{-\beta(\kappa)}^{-}\right\}$where $\eta_{\beta}^{ \pm}:=\frac{2}{\pi} \ln \left(x_{\beta}^{ \pm}\right)$, which is consistent with [13, § 4.1]. Since $x_{\beta}^{+} x_{\beta}^{-}=1$, we have $\eta_{\beta}^{-}=-\eta_{\beta}^{+}$.

Example $30(\phi=\pi / 3) . P_{\beta}^{(1,3)}(x)=x^{4}+x^{2}+1+\beta\left(x^{3}+x\right)$. Since the roots of $P_{\beta}^{(1,3)}$ are stable by inversion, we seek $\left(\xi_{1}, \xi_{2}\right) \in \mathbb{C}^{*} \times \mathbb{C}^{*}$ such that $P_{\beta}^{(1,3)}(x)=\left(x-\xi_{1}\right)\left(x-1 / \xi_{1}\right)\left(x-\xi_{2}\right)\left(x-1 / \xi_{2}\right)$, which is equivalent to

$$
\frac{1}{\xi_{2}}+\xi_{2}+\frac{1}{\xi_{1}}+\xi_{1}=-\beta,\left(\frac{1}{\xi_{2}}+\xi_{2}\right) \times\left(\frac{1}{\xi_{1}}+\xi_{1}\right)=-1
$$

This yields the expression of the four roots of $P_{\beta}^{(1,3)}$ :

$$
\left\{x_{\beta}^{ \pm, i}\right\}_{i \in\{+,-\}}=\left\{\frac{1}{2}\left(A_{i} \pm \sqrt{A_{i}^{2}-4}\right)\right\}_{i \in\{+,-\}}, \text { where } A_{ \pm}:=\frac{-\beta \pm \sqrt{\beta^{2}+4}}{2},
$$

so that $\breve{H}_{\phi}^{\mathrm{e}}(\kappa)=\left\{\eta_{\beta(\kappa)}^{ \pm, i}\right\}_{i \in\{+,-\}}$ and $\breve{H}_{\phi}^{\mathrm{v}}(\kappa)=\left\{\eta_{-\beta(\kappa)}^{ \pm, i}\right\}_{i \in\{+,-\}}$, where $\eta_{\beta}^{ \pm, i}:=\frac{3}{\pi} \ln \left(x_{\beta}^{ \pm, i}\right)$. Note that since $x_{\beta}^{+, \pm} x_{\beta}^{-, \pm}=1$, we have $\eta_{\beta}^{-, \pm}=-\eta_{\beta}^{+, \pm}$.

\section{Comments on the proposed definition of complex plasmonic resonances}

This appendix discusses the proposed definition of $\mathrm{CP}$ resonances (Definition 17). Let us first recall that the resolvent of a differential operator $\mathcal{A}$ is an operator-valued analytic function $(\mathbb{C} \backslash \sigma) \ni \lambda \mapsto R(\lambda)$ (typically $\lambda=\omega$ or $\lambda=\omega^{2}$ ), which is singular at the spectrum $\sigma \subset \mathbb{C}$. If $\mathcal{A}$ has a non-empty and non-countable continuous spectrum $\sigma_{c}$, then $R$ may be multivalued, i.e. it may admit at least one analytic extension $\tilde{R}$ across $\sigma_{c}$ that differs from $R$, so that $\sigma_{c}$ is effectively a branch cut of $R$. This commonly occurs in scattering problems.

In this paper, the denomination "resonance" is used in the sense encountered in scattering; in particular, "resonance" is not synonymous with "eigenvalue". The operator-theoretic definition of resonances is as follows [36, 37] [64] [65]: for a problem with a multivalued resolvent operator, eigenvalues are poles of the principal branch, while complex resonances are poles of the other branche(s). For example, a single degree-of-freedom oscillator has eigenvalues (complex if damped) but no complex resonances, since the resolvent operator is not multivalued. By contrast, 
in scattering, the resolvent operator that gives the outgoing solution is multivalued since the spatial domain is unbounded: there can be both eigenvalues and complex resonances. An eigenvalue is associated with a finite-energy eigenfunction, while a complex resonance (also "complex scattering resonance" or "complex scattering pole") is associated with a complex resonance function (also "quasi-normal mode"), whose energy is infinite.

This operator-theoretic approach could be followed to define CP resonances. The "outgoing resolvent operator" $R_{\text {out }}$ would be defined using an inverse Mellin transform around the corner [38]. The map $\kappa \mapsto R_{\text {out }}(\kappa)$, naturally defined in $\mathbb{C}^{+}$would have branch points $\kappa_{\phi}, \kappa_{\phi}^{-1}$, and -1 . As such, it would admit at least three different meromorphic continuations to $\mathbb{C}^{-}: R_{0}$ (continuation across $\mathbb{R} \backslash \overline{I_{c}}$ ), $R^{\mathfrak{e}}$ (continuation across $I_{c}^{\mathfrak{e}}$ ), and $R^{\mathfrak{v}}$ (continuation across $I_{c}^{\mathfrak{p}}$ ). Even-critical CP resonances would then be poles of $R^{\mathrm{e}}$ in $\mathbb{C}^{-}$.

The approach we have followed in this paper is to define CP resonances through their local expansions at $r=0$, which only requires the knowledge of the corner dispersion relations (7). This approach is commonly followed in the scattering literature, where complex scattering resonance functions are introduced as solutions of the time-harmonic eigenproblem that are exponentially growing at infinity [53] [51] [60]. This approach suggests that to compute complex resonances this exponential growth must be dealt with, which is the purpose of complex scaling.

\section{Point spectrum of elliptical particles}

Proposition 31. Let $\Omega_{m}, \Omega$, and $\Omega_{d}$ be given by (35) with $\overline{\Omega_{m}} \subset \Omega$. If $\partial \Omega_{m}$ and $\partial \Omega$ are not circles, then the eigenvalues of the PEP $(1,2)$ with a Dirichlet boundary condition on $\partial \Omega$ are given by

$$
\kappa_{0}^{\mathfrak{e}}=0, \kappa_{n}^{\mathfrak{e}}=-\tanh \left[n \mu_{m}\right] \tanh \left[n\left(\mu_{d}-\mu_{m}\right)\right], \kappa_{n}^{\mathfrak{o}}=-\frac{\tanh \left[n\left(\mu_{d}-\mu_{m}\right)\right]}{\tanh \left[n \mu_{m}\right]} \quad\left(n \in \mathbb{N}^{*}\right),
$$

where $r_{m} \in(0,1)$ (resp. $\left.r_{d} \in(0,1)\right)$ is the aspect ratio of $\partial \Omega_{m}$ (resp. $\partial \Omega$ ) and $\mu=\operatorname{arctanh} r$. Eigenfunctions associated with $\kappa_{n}^{\mathrm{e}}\left(\right.$ resp. $\left.\kappa_{n}^{\mathfrak{o}}\right)$ are even (resp. odd) with respect to the major axis.

For any $n \in \mathbb{N}, \kappa_{n}^{\mathrm{e}} \in(-1,0]$ while for any $n \in \mathbb{N}^{*}, \kappa_{n}^{\mathfrak{o}} \in(-1,0)$ when $\mu_{d}<2 \mu_{m}$ and $\kappa_{n}^{\mathfrak{o}}<-1$ when $\mu_{d}>2 \mu_{m}$. In the limit $\mu_{d} \rightarrow \infty$ we recover the eigenvalues of the NP operator on $\partial \Omega_{m}$ [66, Eq. (4.13)].

Proof. Without loss of generality, we assume that $\Omega_{m}$ is such that $a_{m}>b_{m}$ so that its focal distance is $c=\sqrt{a_{m}^{2}-b_{m}^{2}}$. Let $(\mu, \theta)$ be the elliptic coordinates associated with the focal points of $\partial \Omega_{m}$ (see Figure $8 \mathrm{~b}$ ):

$$
x=c \cosh \mu \cos \theta, y=c \sinh \mu \sin \theta \quad(\mu>0, \theta \in(-\pi, \pi)) .
$$

Note that $\{\mu=0, \theta \in(-\pi, \pi)\}$ corresponds to the segment $(-c,+c) \times\{0\}$. In elliptic coordinates, the PEP reads

$$
\frac{1}{c^{2}} \frac{1}{\sinh ^{2} \mu+\sin ^{2} \theta}\left[\partial_{\mu}^{2} u(\mu, \theta)+\partial_{\theta}^{2} u(\mu, \theta)\right]=0 \quad\left(\mu \in\left(0, \mu_{m}\right) \cup\left(\mu_{m}, \mu_{d}\right)\right),
$$

with boundary conditions

$$
\begin{array}{ll}
u(\mu,-\pi)=u(\mu, \pi) & \text { (A) } \partial_{\theta} u(\mu,-\pi)=\partial_{\theta} u(\mu, \pi) \\
u_{\mid \Omega_{d}}\left(\mu_{m}, \cdot\right)=u_{\mid \Omega_{m}}\left(\mu_{m}, \cdot\right) & \text { (C) } \kappa \partial_{\mu} u_{\mid \Omega_{d}}\left(\mu_{m}, \cdot\right)=\partial_{\mu} u_{\mid \Omega_{m}}\left(\mu_{m}, \cdot\right) \\
u\left(\mu_{d}, \cdot\right)=0 . & \text { (E) }
\end{array}
$$

Injecting $u(\mu, \theta)=\varphi(\mu) \Phi(\theta)$ into the PEP leads to

$$
\varphi^{\prime \prime}(\mu)=n^{2} \varphi(\mu), \Phi(\theta)=C_{1} \cos (n \theta)+D_{1} \sin (n \theta) \quad(n \in \mathbb{Z}),
$$

where the constraint $n \in \mathbb{Z}$ follows from the periodicity conditions (A,B). If $n=0$ and $\kappa \neq 0$, then $u=0$. If $n=0$ and $\kappa=0$, then $u(\mu, \theta) \propto \mathbb{1}_{\Omega_{m}}+\frac{\mu-\mu_{d}}{\mu_{m}-\mu_{d}} \mathbb{1}_{\Omega_{d}}$. If $n \neq 0$, we seek $\varphi$ as

$$
\varphi(\mu)= \begin{cases}A_{2} \cosh \left(n\left(\mu-\mu_{d}\right)\right)+B_{2} \sinh \left(n\left(\mu-\mu_{d}\right)\right) & \left(\mu \in\left(\mu_{m}, \mu_{d}\right)\right) \\ A_{1} \cosh (n \mu)+B_{1} \sinh (n \mu) & \left(\mu \in\left(0, \mu_{m}\right)\right) .\end{cases}
$$


(Even modes, $\left.D_{1}=0\right)$ Conditions $(\mathrm{C}, \mathrm{D}, \mathrm{E})$ and $\varphi^{\prime}(0)=0$ yield $A_{2}=B_{1}=0$ and $\left[\begin{array}{cc}\cosh \left(n \mu_{m}\right) & -\sinh \left(n\left(\mu_{m}-\mu_{d}\right)\right) \\ n \sinh \left(n \mu_{m}\right) & -n \kappa \cosh \left(n\left(\mu_{m}-\mu_{d}\right)\right)\end{array}\right]\left[\begin{array}{c}A_{1} \\ B_{2}\end{array}\right]=0, \varphi_{n}^{\mathrm{e}}(\mu)=B_{2} \begin{cases}\sinh \left(n\left(\mu-\mu_{d}\right)\right) & \left(\mu \in\left(\mu_{m}, \mu_{d}\right)\right) \\ \frac{\sinh \left(n\left(\mu_{m}-\mu_{d}\right)\right)}{\cosh \left(n \mu_{m}\right)} \cosh (n \mu) & \left(\mu \in\left(0, \mu_{m}\right)\right) .\end{cases}$

(Odd modes, $C_{1}=0$ ) Conditions $(\mathrm{C}, \mathrm{D}, \mathrm{E})$ and $\varphi(0)=0$ yield $A_{1}=A_{2}=0$ and

$$
\left[\begin{array}{cc}
\sinh \left(n \mu_{m}\right) & -\sinh \left(n\left(\mu_{m}-\mu_{d}\right)\right) \\
n \cosh \left(n \mu_{m}\right) & -n \kappa \cosh \left(n\left(\mu_{m}-\mu_{d}\right)\right)
\end{array}\right]\left[\begin{array}{c}
B_{1} \\
B_{2}
\end{array}\right]=0, \varphi_{n}^{\mathrm{o}}(\mu)=B_{2} \begin{cases}\sinh \left(n\left(\mu-\mu_{d}\right)\right) & \left(\mu \in\left(\mu_{m}, \mu_{d}\right)\right) \\
\frac{\sinh \left(n\left(\mu_{m}-\mu_{d}\right)\right)}{\sinh \left(n \mu_{m}\right)} \sinh (n \mu) & \left(\mu \in\left(0, \mu_{m}\right)\right) .\end{cases}
$$

\section{References}

[1] D. Grieser, The plasmonic eigenvalue problem, Reviews in Mathematical Physics 26 (03) (2014) 1450005. doi:10.1142/ S0129055X14500056.

[2] E. D. Palik, Handbook of Optical Constants of Solids, Academic Press, London, 1998.

[3] D. Jackson, Classical electrodynamics, 3rd Edition, John Wiley \& Sons, Hoboken, New Jersey, 1999.

[4] H. Ammari, Y. Deng, P. Millien, Surface plasmon resonance of nanoparticles and applications in imaging, Archive for Rational Mechanics and Analysis 220 (1) (2016) 109-153. doi:10.1007/s00205-015-0928-0.

[5] K. Ando, H. Kang, H. Liu, Plasmon resonance with finite frequencies: a validation of the quasi-static approximation for diametrically small inclusions, SIAM Journal on Applied Mathematics 76 (2) (2016) 731-749. doi : 10.1137/15M1025943.

[6] S. Maier, Plasmonics fundamentals and applications, Springer, New York, 2007.

[7] P. Markos, C. Soukoulis, Wave propagation from electrons to photonic crystals and left-handed materials, Princeton University Press, Princeton, New Jersey, 2008.

[8] D. Sarid, W. Challener, Modern Introduction to Surface Plasmons, Cambridge University Press, Cambridge, 2010.

[9] A. V. Zayats, I. I. Smolyaninov, A. A. Maradudin, Nano-optics of surface plasmon polaritons, Physics Reports 408 (3) (2005) $131-314$. doi:10.1016/j.physrep.2004.11.001.

[10] D. K. Gramotnev, S. I. Bozhevolnyi, Plasmonics beyond the diffraction limit, Nature photonics 4 (2) (2010) 83-91. doi:10.1038/nphoton. 2009.282.

[11] J. Pendry, Negative refraction, Contemporary Physics 45 (3) (2004) 191-202. doi : 10.1080/00107510410001667434.

[12] S. A. Ramakrishna, Physics of negative refractive index materials, Reports on Progress in Physics 68 (2) (2005) 449-521. doi:10.1088/ 0034-4885/68/2/R06.

[13] A.-S. Bonnet-Ben Dhia, L. Chesnel, X. Claeys, Radiation condition for a non-smooth interface between a dielectric and a metamaterial, Mathematical Models and Methods in Applied Sciences 23 (09) (2013) 1629-1662. doi:10.1142/S0218202513500188.

[14] A.-S. Bonnet-Ben Dhia, L. Chesnel, P. Ciarlet, T-coercivity for scalar interface problems between dielectrics and metamaterials, ESAIM: Mathematical Modelling and Numerical Analysis 46 (6) (2012) 1363-1387. doi:10.1051/m2an/2012006.

[15] E. Bonnetier, H. Zhang, Characterization of the essential spectrum of the Neumann-Poincaré operator in 2D domains with corner via Weyl sequences, arXiv preprint arXiv:1702.08127 (2017).

[16] C. Hazard, S. Paolantoni, Spectral analysis of polygonal cavities containing a negative-index material, Annales Henri Lebesgue 3 (2020) 1161-1193. doi:10.5802/ahl.58.

[17] A.-S. Bonnet-Ben Dhia, C. Carvalho, L. Chesnel, P. Ciarlet, On the use of perfectly matched layers at corners for scattering problems with sign-changing coefficients, Journal of Computational Physics 322 (2016) 224-247. doi : 10.1016/j.jcp.2016.06.037.

[18] M. I. Stockman, S. V. Faleev, D. J. Bergman, Localization versus delocalization of surface plasmons in nanosystems: Can one state have both characteristics?, Physical Review Letters 87 (2001) 167401. doi:10.1103/PhysRevLett.87.167401.

[19] G. B. Folland, Introduction to Partial Differential Equations, 2nd Edition, Princeton University Press, Princeton, New Jersey, 1995.

[20] D. Colton, R. Kress, Inverse Acoustic and Electromagnetic Scattering Theory, Springer-Verlag, Berlin, 1998.

[21] F. Ouyang, M. Isaacson, Surface plasmon excitation of objects with arbitrary shape and dielectric constant, Philosophical Magazine B 60 (4) (1989) 481-492. doi:10.1080/13642818908205921.

[22] H. Kang, J. Seo, Recent progress in the inverse conductivity problem with single measurement, in: G. Nakamura, J. Seo, M. Yamamoto (Eds.), Inverse problems and related topics, Chapman \& Hall/CRC, Boca Raton, 2000, pp. 69-80.

[23] H. Ammari, P. Millien, M. Ruiz, H. Zhang, Mathematical analysis of plasmonic nanoparticles: The scalar case, Archive for Rational Mechanics and Analysis 224 (2) (2017) 597-658. doi : 10.1007/s00205-017-1084-5.

[24] I. D. Mayergoyz, D. R. Fredkin, Z. Zhang, Electrostatic (plasmon) resonances in nanoparticles, Physical Review B 72 (2005) 155412. doi:10.1103/PhysRevB.72.155412.

[25] J. Helsing, A. Karlsson, On a Helmholtz transmission problem in planar domains with corners, Journal of Computational Physics 371 (2018) 315-332. doi:10.1016/j.jcp.2018.05.044.

[26] H. Ammari, Y. Chow, K. Liu, J. Zou, Optimal shape design by partial spectral data, SIAM Journal on Scientific Computing 37 (6) (2015) B855-B883. doi:10.1137/130942498.

[27] K. Ando, H. Kang, Analysis of plasmon resonance on smooth domains using spectral properties of the Neumann-Poincaré operator, Journal of Mathematical Analysis and Applications 435 (1) (2016) 162-178. doi:10.1016/j . jmaa.2015.10.033.

[28] H. Kang, M. Lim, S. Yu, Spectral resolution of the Neumann-Poincaré operator on intersecting disks and analysis of plasmon resonance, Archive for Rational Mechanics and Analysis 226 (1) (2017) 83-115. doi:10.1007/s00205-017-1129-9. 
[29] K.-M. Perfekt, M. Putinar, Spectral bounds for the Neumann-Poincaré operator on planar domains with corners, Journal d'Analyse Mathématique 124 (1) (2014) 39-57. doi:10.1007/s11854-014-0026-5.

[30] H. Ammari, B. Fitzpatrick, H. Kang, M. Ruiz, S. Yu, H. Zhang, Mathematical and computational methods in photonics and phononics, Vol. 235, American Mathematical Society, Providence, Rhode Island, 2018.

[31] K.-M. Perfekt, M. Putinar, The essential spectrum of the Neumann-Poincaré operator on a domain with corners, Archive for Rational Mechanics and Analysis 223 (2) (2017) 1019-1033. doi:10.1007/s00205-016-1051-6.

[32] Y. Luo, J. B. Pendry, A. Aubry, Surface plasmons and singularities, Nano letters 10 (10) (2010) 4186-4191. doi:10.1021/nl102498s.

[33] K.-M. Perfekt, Plasmonic eigenvalue problem for corners: Limiting absorption principle and absolute continuity in the essential spectrum, Journal de Mathématiques Pures et Appliquées 145 (2021) 130-162. doi:https://doi.org/10.1016/j .matpur.2020.07.001.

[34] J. Helsing, H. Kang, M. Lim, Classification of spectra of the Neumann-Poincaré operator on planar domains with corners by resonance, Annales de l'Institut Henri Poincare (C) Non Linear Analysis 34 (4) (2017) 991-1011. doi : 10.1016/j . anihpc . 2016.07.004.

[35] W. Li, S. P. Shipman, Embedded eigenvalues for the Neumann-Poincaré operator, Journal of Integral Equations and Applications 31 (4) (2019) 505-534. doi:10.1216/JIE-2019-31-4-505.

[36] M. Zworski, Resonances in physics and geometry, Notices of the AMS 46 (3) (1999) 319-328.

[37] M. Zworski, Mathematical study of scattering resonances, Bulletin of Mathematical Sciences 7 (1) (2017) 1-85. doi:10.1007/ s13373-017-0099-4.

[38] M. Dauge, B. Texier, Problèmes de transmission non coercifs dans des polygones, hal-00562329 (1997).

[39] V. A. Kondrat'ev, O. A. Oleinik, Boundary-value problems for partial differential equations in non-smooth domains, Russian Mathematical Surveys 38 (2) (1983) 1-86. doi:10.1070/rm1983v038n02abeh003470.

[40] S. Nazarov, B. Plamenevsky, Elliptic problems in domains with piecewise smooth boundaries, Walter de Gruyter, Berlin, 1994.

[41] M. Costabel, M. Dauge, Singularities of electromagnetic fields in polyhedral domains, Archive for Rational Mechanics and Analysis 151 (2000) 221-276. doi:10.1007/s002050050197.

[42] V. Kozlov, V. Maz'ya, J. Rossman, Spectral problems asociated with corner singularities of solutions to elliptic equations, American Mathematical Society, Providence, Rhode Island, 2001.

[43] T. W. Gamelin, Complex Analysis, Springer-Verlag, New York, 2001.

[44] W. Ebeling, Functions of Several Complex Variables and Their Singularities, American Mathematical Society, Providence, Rhode Island, 2007.

[45] J. Aguilar, J. M. Combes, A class of analytic perturbations for one-body Schrödinger hamiltonians, Communications in Mathematical Physics 22 (4) (1971) 269-279. doi:10.1007/BF01877510.

[46] E. Balslev, J. M. Combes, Spectral properties of many-body Schrödinger operators with dilatation-analytic interactions, Communications in Mathematical Physics 22 (4) (1971) 280-294. doi:10.1007/BF01877511.

[47] P. Hislop, I. Sigal, Introduction to Spectral Theory with Applications to Schrödinger Operators, Springer, New York, 1996.

[48] J.-P. Berenger, A perfectly matched layer for the absorption of electromagnetic waves, Journal of Computational Physics 114 (2) (1994) 185-200. doi:10.1006/jcph.1994.1159.

[49] W. C. Chew, W. H. Weedon, A 3D perfectly matched medium from modified Maxwell's equations with stretched coordinates, Microwave and Optical Technology Letters 7 (13) (1994) 599-604. doi:10.1002/mop.4650071304.

[50] C. M. Rappaport, Perfectly matched absorbing boundary conditions based on anisotropic lossy mapping of space, IEEE Microwave and Guided Wave Letters 5 (3) (1995) 90-92. doi : 10.1109/75.366463.

[51] S. Kim, J. Pasciak, The computation of resonances in open systems using a perfectly matched layer, Mathematics of Computation 78 (267) (2009) 1375-1398. doi:10.1090/S0025-5718-09-02227-3.

[52] S. Dyatlov, M. Zworski, Mathematical Theory of Scattering Resonances, Version 0.1, 2018.

[53] Y. Duan, W. Koch, C. M. Linton, M. McIver, Complex resonances and trapped modes in ducted domains, Journal of Fluid Mechanics 571 (2007) 119-147. doi:10.1017/S0022112006003259.

[54] S. Hein, W. Koch, L. Nannen, Fano resonances in acoustics, Journal of Fluid Mechanics 664 (2010) 238-264. doi:10.1017/ S0022112010003757.

[55] A. Ern, J.-L. Guermond, Theory and Practice of Finite Elements, Springer-Verlag, New York, 2004. doi : 978-1-4419-1918-2.

[56] M. Lenoir, Optimal isoparametric finite elements and error estimates for domains involving curved boundaries, SIAM Journal on Numerical Analysis 23 (3) (1986) 562-580. doi:10.1137/0723036.

[57] R. B. Lehoucq, D. C. Sorensen, C. Yang, ARPACK Users' Guide, Society for Industrial and Applied Mathematics, 1998. doi :10.1137/1. 9780898719628.

[58] C. Carvalho, L. Chesnel, P. Ciarlet, Eigenvalue problems with sign-changing coefficients, Comptes Rendus Mathematique 355 (6) (2017) 671 - 675. doi:10.1016/j.crma.2017.05.002.

[59] A.-S. Bonnet-Ben Dhia, C. Carvalho, P. Ciarlet, Mesh requirements for the finite element approximation of problems with sign-changing coefficients, Numerische Mathematik 138 (4) (2018) 801-838. doi :10.1007/s00211-017-0923-5.

[60] L. Nannen, M. Wess, Computing scattering resonances using perfectly matched layers with frequency dependent scaling functions, BIT Numerical Mathematics 58 (2) (2018) 373-395. doi:10.1007/s10543-018-0694-0.

[61] J. Helsing, K.-M. Perfekt, The spectra of harmonic layer potential operators on domains with rotationally symmetric conical points, Journal de Mathématiques Pures et Appliquées 118 (2018) 235-287. doi:10.1016/j.matpur.2017.10.012.

[62] W. Li, K.-M. Perfekt, S. P. Shipman, Infinitely many embedded eigenvalues for the Neumann-Poincaré operator in 3D (2020). arXiv: 2009.04371

[63] W. Rudin, Real and Complex Analysis, 3rd Edition, McGraw-Hill Book Company, New York, 1986.

[64] M. Lenoir, M. Vullierme-Ledard, C. Hazard, Variational formulations for the determination of resonant states in scattering problems, SIAM Journal on Mathematical Analysis 23 (3) (1992) 579-608. doi : 10.1137/0523030.

[65] A. Aslanyan, L. Parnovski, D. Vassiliev, Complex resonances in acoustic waveguides, The Quarterly Journal of Mechanics and Applied Mathematics 53 (3) (2000) 429-447. doi : 10.1093/qjmam/53.3.429.

[66] J. F. Ahner, Some spectral properties of an integral operator in potential theory, Proceedings of the Edinburgh Mathematical Society 29 (3) (1986) 405-411. doi:10.1017/S0013091500017843. 